\title{
PRACTICE GUIDELINE FOR THE Treatment of Patients With HIV/AIDS
}

\section{WORK GROUP ON HIV/AIDS}

J. Stephen McDaniel, M.D., Chair

Larry Brown, M.D.

Francine Cournos, M.D.

Marshall Forstein, M.D.

Karl Goodkin, M.D., Ph.D.

Constantine Lyketsos, M.D., M.H.S.

Joyce Y. Chung, M.D. (Consultant)

Originally published in November 2000. This guideline is more than 5 years old and has not yet been updated to ensure that it reflects current knowledge and practice. In accordance with national standards, including those of the Agency for Healthcare Research and Quality's National Guideline Clearinghouse (http://www.guideline.gov/), this guideline can no longer be assumed to be current. The April 2006 Guideline Watch associated with this guideline provides additional information that has become available since publication of the guideline, but it is not a formal update of the guideline. 


\section{AMERICAN PSYCHIATRIC ASSOCIATION STEERING COMMITTEE ON PRACTICE GUIDELINES}

John S. McIntyre, M.D., Chair

Sara C. Charles, M.D., Vice-Chair

Daniel J. Anzia, M.D.

Ian A. Cook, M.D.

Molly T. Finnerty, M.D.

Bradley R. Johnson, M.D.

James E. Nininger, M.D.

Paul Summergrad, M.D.

Sherwyn M. Woods, M.D., Ph.D.

Joel Yager, M.D.

\section{AREA AND COMPONENT LIAISONS}

Robert Pyles, M.D. (Area I)

C. Deborah Cross, M.D. (Area II)

Roger Peele, M.D. (Area III)

Daniel J. Anzia, M.D. (Area IV)

John P. D. Shemo, M.D. (Area V)

Lawrence Lurie, M.D. (Area VI)

R. Dale Walker, M.D. (Area VII)

Mary Ann Barnovitz, M.D.

Sheila Hafter Gray, M.D.

Sunil Saxena, M.D.

Tina Tonnu, M.D.

\section{STAFF}

Robert Kunkle, M.A., Senior Program Manager

Amy B. Albert, B.A., Assistant Project Manager

Laura J. Fochtmann, M.D., Medical Editor

Claudia Hart, Director, Department of Quality Improvement and

Psychiatric Services

Darrel A. Regier, M.D., M.P.H., Director, Division of Research 


\section{CONTENTS}

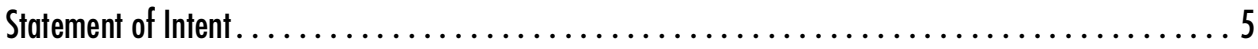

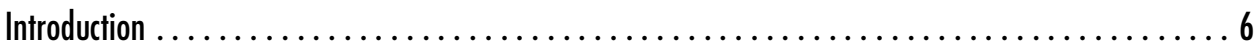

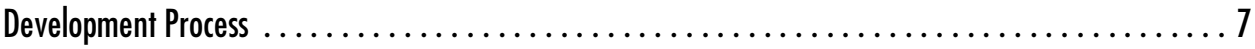

Part A: Background Information and Treatment Recommendations for

Patients With HIV/AIDS......................................

I. Summary of Treatment Recommendations............................?

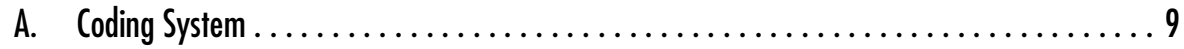

B. General Considerations..................................... 9

II. Disease Definition, Epidemiology, and Natural History..................... 12

A. Basic Facts About HIV Infection, AIDS, and Treatments .................. 12

B. Associated Features of Neuropsychiatric and Psychosocial Importance.............. 20

C. Prevalence of Comorbid Psychiatric Conditions........................ 26

III. Formulation and Implementation of a Treatment Plan ........................ 27

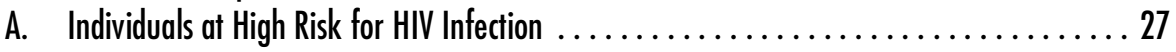

B. HIV-Infected Individuals ................................... 31

IV. Clinical and Environmental Features Influencing Treatment $\ldots \ldots \ldots \ldots \ldots \ldots \ldots \ldots$

A. Sociodemographic Variables .................................... 39

B. Clinical Features....................................... 42

C. Special Treatment Situations ................................ 45

Part B: Review and Synthesis of Available Evidence .................. 46

V. Data Regarding Prevention for Individuals at High Risk for HIV Infection . . . . . . . . . . . . 46

A. HIV-Related Risk Assessment of Behavior ................................ 47

B. HIV Antibody Testing. ................................... 47

C. Interventions to Reduce HIV Risk Behavior .......................... 47

D. Reducing Maternal-Fetal Transmission . ............................. 49

E. Postexposure Prophylaxis.................................... 50

F. Special Psychiatric Populations ................................ 50 
VI. Data Regarding Psychiatric Treatments for Individuals With HIV Infection .............5I

A. Access to Services ......................................... 52

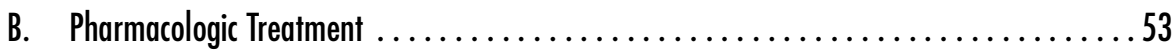

C. Psychosocial Treatment ......................................62

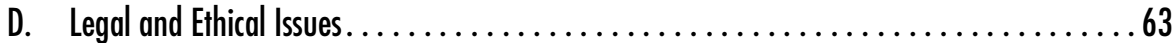

E. Adherence to Treatment ....................................64

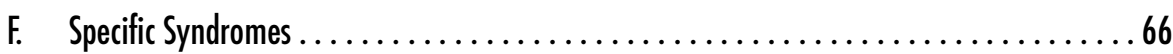

Part C: Future Research Needs .................................. 77

Individuals and Organizations That Submitted Comments ........................ 81

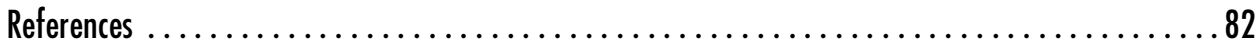




\section{STATEMENT OF INTENT}

The American Psychiatric Association (APA) Practice Guidelines are not intended to be construed or to serve as a standard of medical care. Standards of medical care are determined on the basis of all clinical data available for an individual patient and are subject to change as scientific knowledge and technology advance and practice patterns evolve. These parameters of practice should be considered guidelines only. Adherence to them will not ensure a successful outcome for every individual, nor should they be interpreted as including all proper methods of care or excluding other acceptable methods of care aimed at the same results. The ultimate judgment regarding a particular clinical procedure or treatment plan must be made by the psychiatrist in light of the clinical data presented by the patient and the diagnostic and treatment options available.

This practice guideline has been developed by psychiatrists who are in active clinical practice. In addition, some contributors are primarily involved in research or other academic endeavors. It is possible that through such activities some contributors, including work group members and reviewers, have received income related to treatments discussed in this guideline. A number of mechanisms are in place to minimize the potential for producing biased recommendations due to conflicts of interest. Work group members are selected on the basis of their expertise and integrity. Any work group member or reviewer who has a potential conflict of interest that may bias (or appear to bias) his or her work is asked to disclose this to the Steering Committee on Practice Guidelines and the work group. Iterative guideline drafts are reviewed by the Steering Committee, other experts, allied organizations, APA members, and the APA Assembly and Board of Trustees; substantial revisions address or integrate the comments of these multiple reviewers. The development of the APA practice guidelines is not financially supported by any commercial organization.

More detail about mechanisms in place to minimize bias is provided in a document available from the APA Department of Quality Improvement and Psychiatric Services, "APA Guideline Development Process."

This practice guideline was approved in July 2000 and published in November 2000. 


\section{INTRODUCTION}

This practice guideline seeks to summarize data and specific forms of treatment regarding the care of patients with HIV/AIDS. The purpose of this guideline is to assist the psychiatrist in caring for a patient with HIV/AIDS.

Psychiatrists care for patients with HIV/AIDS in many different settings and serve a variety of functions. In many cases, a psychiatrist will serve as a consultant to the patient's primary care physician. This guideline reviews the treatment that patients with HIV/AIDS may need. The psychiatrist should either provide or advocate for the appropriate treatments.

To share feedback on this or other published APA practice guidelines, a form is available at http://www.psych.org/psych_pract/pg/reviewform.cfm. 


\section{DEVELOPMENT PROCESS}

This document is a practical guide to the management of patients — primarily adults over the age of 18-with HIV/AIDS and represents a synthesis of current scientific knowledge and rational clinical practice. This guideline strives to be as free as possible of bias toward any theoretical approach to treatment.

This practice guideline was developed under the auspices of the Steering Committee on Practice Guidelines. The process is detailed in a document available from the APA Department of Quality Improvement and Psychiatric Services: the "APA Guideline Development Process." Key features of the process include the following:

- a comprehensive literature review (description follows) and development of evidence tables;

- initial drafting by a work group that included psychiatrists with clinical and research expertise in HIV/AIDS;

- the production of multiple drafts with widespread review, in which 14 organizations and over 60 individuals submitted significant comments;

- approval by the APA Assembly and Board of Trustees; and

- planned revisions at regular intervals.

A computerized search of the relevant literature from MEDLINE, PsycINFO, EMBASE, and AIDSLINE was conducted.

The first literature search was conducted by using MEDLINE for the period from 1966 to June 1998 and used the key words "acquired immunodeficiency syndrome," "HIV," "dementia," "mood disorders," "anxiety," "sleep," "depression mania," "substance use," and "adjustment disorders."

The literature search conducted by using PsycINFO covered the period from 1967 to March 1998 and used the key words "acquired immune deficiency syndrome," "adjustment disorders," "human immunodeficiency virus," "dementia," and "mood disorders."

The literature search conducted by using EMBASE covered the period from 1980 to 1998 and used the key words "acquired immune deficiency syndrome," "adjustment disorders," "human immunodeficiency virus," "manic depressive psychosis," "depression," and "mood disorders."

The literature search conducted by using AIDSLINE covered the period from 1980 to 1998 and used the key words "acquired immunodeficiency syndrome," "HIV," "adjustment disorders," "psychotherapy," "dementia," "treatment," "therapy," "therapeutic," and "mood disorders."

An additional literature search was conducted by using MEDLINE for the period from 1990 to 1999 and used the key words "correctional settings," "jail," "women," "minorities," and "suicide."

Additional, less formal, literature searches were conducted by APA staff and individual members of the work group on HIV/AIDS. 


\section{PART A:}

\section{BACKGROUND INFORMATION AND TREATMENT RECOMMENDATIONS FOR PATIENTS WITH HIV/AIDS}

\section{SUMMARY OF TREATMENT RECOMMENDATIONS}

The following executive summary is intended to provide an overview of the organization and scope of recommendations in this practice guideline. Because psychiatric treatment of patients with HIV/AIDS requires the consideration of many factors, it cannot be adequately reviewed in a brief summary. The reader is encouraged to consult the relevant portions of the guideline when specific treatment recommendations are sought. This summary is not intended to stand on its own.

\section{A. CODING SYSTEM}

Each recommendation is identified as falling into one of three categories of endorsement, indicated by a bracketed Roman numeral following the statement. The three categories represent varying levels of clinical confidence regarding the recommendation:

[I] Recommended with substantial clinical confidence.

[II] Recommended with moderate clinical confidence.

[III] May be recommended on the basis of individual circumstances.

\section{B. GENERAL CONSIDERATIONS}

All psychiatrists need to have an adequate fund of knowledge about HIV/AIDS. Because scientific knowledge regarding virology, prevention, and interventions to treat HIV infection advances rapidly, this guideline attempts to summarize current knowledge and principles of treatment with the expectation that updated information will be needed. For this reason, several pertinent web site addresses have been included both in the text and in an appendix at the end of the guideline. The reader is encouraged to use the web sites to search for additional or more current information that will supplement the information provided in this guideline. Section II provides information about the following: the pathophysiology and virology of HIV, the epidemiology of HIV/AIDS, transmission of HIV, pathogenesis of HIV, assessment and staging of HIV disease, treatment for HIV infection, impact of HIV on the central nervous system, neuropsychiatric clinical syndromes, differential diagnosis of neuropsychiatric syndromes, pediatric HIV/AIDS syndromes, and prevalence of comorbid psychiatric conditions. 
Section III serves as a guide for formulating and implementing a treatment plan, and its outline is mirrored in Sections V and VI, in which the data regarding prevention and treatment, respectively, are provided. Readers may find it useful to first read Section III and then refer to Sections V and VI for the scientific evidence on which the treatment recommendations have been made.

Because there are many clinical and environmental features that may influence treatment, Section IV provides additional information on sociodemographic variables such as race/ethnicity, sexual orientation, gender, age, economic factors, and setting (urban versus rural). Specific clinical topics of suicidality, bereavement, axis II disorders, and psychoneuroimmunology are also covered. Last, information about special treatment situations such as alternative/complementary treatments, institutional settings, and health care clinician issues is included.

\section{Psychiatric management of individuals at high risk for HIV infection}

Psychiatrists may encounter patients in the course of their clinical work who are at risk for acquiring HIV infection. Optimum management of patients at high risk for HIV infection uses a wide range of psychiatric skills such as comprehensive diagnostic evaluations, assessment of possible medical causes of new-onset symptoms, initiation of specific treatment interventions, and a keen understanding of psychodynamic issues [I]. Patients with substance use disorders, patients with severe mental illness, and victims of sexual abuse/crimes have specific risks for becoming infected with HIV [I]. Psychiatric management of individuals who, because of specific behaviors, are at risk for HIV infection involves obtaining a risk history, considering the need for HIV antibody testing, initiating risk reduction strategies, and initiating postexposure prophylaxis with antiretroviral medication when indicated. A psychiatrist will not be aware of a patient's risk for HIV infection unless risk behavior is accurately assessed [I]. Psychiatrists should be knowledgeable about which specific risk behaviors are more likely to result in HIV transmission [I]. Psychiatric units or individual practitioners who conduct HIV testing should be aware of their obligation to provide the necessary pre- and posttest counseling [I]. Because successful risk reduction requires more than knowledge of risk, ongoing discussions between patient and psychiatrist can help provide the motivating and skill-building factors that help ensure consistent changes in behavior [II].

Psychiatrists serve as primary clinicians of both medical and psychiatric care in some institutional settings. Thus, administrators of these facilities should formulate policies that support the full range of HIV prevention steps outlined in this guideline [I]. Postexposure prophylaxis is recommended for known occupational exposure, especially percutaneous or mucous membrane exposure, to blood or other body fluids [I]. The American College of Obstetricians and Gynecologists now recommends that an HIV antibody test be offered during annual exams to all women seeking preconception care, not just pregnant women [II].

\section{Psychiatric management of HIV-infected individuals}

Specific tasks that constitute the psychiatric management of patients with HIV/AIDS include the following: establishing and maintaining a therapeutic alliance; collaboration and coordination of care with other mental health and medical providers; diagnosing and treating all associated psychiatric disorders; facilitating adherence to the overall treatment plan; providing education about psychological, psychiatric, and neuropsychiatric disorders; providing risk reduction strategies to further minimize the spread of HIV; maximizing psychological and social/ adaptive functioning; considering the role of religion/spirituality; preparing the patient for issues of disability, death, and dying; and advising significant others/family regarding sources of care and support.

The development of a psychiatric treatment plan for patients with HIV infection requires thoughtful and comprehensive consideration of the biopsychosocial context of the illness [I]. 
When seeing a patient in consultation, it is important to gather history about cognitive or motor symptoms and conduct a mental status screening examination to determine whether neurocognitive deficits are present [I]. Psychiatrists should be knowledgeable about medication side effects and drug interactions of psychotropic agents as well as HIV-related medications in order to provide optimum patient care [I]. Psychiatric treatment of patients with HIV infection should include active monitoring of substance abuse, since it is often associated with risk behaviors that can lead to further transmission of HIV [I]. Adherence is of utmost concern with antiretroviral treatment because the regimens are so unforgiving; even minor deviations from the prescribed regimen can result in viral resistance and permanent loss of efficacy for existing medications [I]. Psychiatrists can play an important role in the promotion of patient adherence, since comorbid psychiatric disorders (e.g., substance abuse or depression) have been shown to adversely affect patient compliance with a complicated treatment regimen [II].

\section{Treatment of psychiatric disorders that result from or are comorbid with HIV infection}

Psychiatric disorders associated with HIV/AIDS should be accurately identified and treated [I]. In adults and children with HIV infection, changes in mental status or the emergence of new psychiatric or cognitive disorders requires ruling out treatable and reversible causes; medical causes are of increasing concern if CD4 counts are low or viral load has begun to rise [I]. The more common diagnoses found in association with HIV/AIDS are dementia and the spectrum of cognitive disorders; delirium; mood disorders; substance use disorders; anxiety disorders; psychotic disorders; adjustment disorders; sleep disorders; disorders occurring in infants, children, and adolescents; and HIV-associated syndromes with psychiatric implications. Both psychopharmacologic and psychotherapeutic treatment strategies are often indicated.

Treatment of HIV-associated dementia consists of intervening with combination antiretroviral therapy that targets the underlying HIV infection, with consideration of whether the agents adequately penetrate the central nervous system (CNS); management of symptoms associated with HIV-associated dementia (e.g., agitation or fatigue) with antipsychotic or stimulant agents, respectively, should be considered [I]. Delirium in the context of HIV infection may often be caused by interactions between the many medications taken by HIV patients. Management of delirium includes the judicious use of antipsychotic medications, with many clinicians choosing atypical agents because of their lower side effect profile; benzodiazepines are relatively contraindicated [II].

The management of disturbances in mood, such as major depression or mania, for patients with HIV infection is similar to that for other patients with medical comorbidity [I]. Choice of an antidepressant or mood-stabilizing agent may be influenced by the antiretroviral regimen in place, and dose adjustments may be necessary if drug-drug interactions are likely. A wide array of antidepressant agents are effective in the treatment of HIV-associated major depression, including newer agents such as the selective serotonin reuptake inhibitors (SSRIs) [I] and medications such as psychostimulants and testosterone [II]. Psychotherapy, particularly interpersonal psychotherapy, either alone or in combination with antidepressant agents, is also an effective treatment for HIV-related depression [II]. Mania associated with HIV infection, particularly late in the course of HIV disease, may be difficult to treat; however, treatment studies suggest that traditional antimanic agents are effective and tolerated [II].

The constellation of other disorders associated with HIV infection requires treatment. Substance use disorders are prevalent among persons with or at risk for HIV infection, and treatment is a high priority [I]. Psychiatrists should be aware that by treating substance abuse, they may well be preventing HIV infection. One component of a comprehensive approach to HIV prevention among injection drug users is access to sterile syringes [I]. Treatment of anxiety and sleep disorders among HIV-infected patients has not been well studied. For patients who are taking protease inhibitors, benzodiazepines are generally contraindicated because of drug-drug 
interactions [II]. Thus, benzodiazepines should be given only as a short-term intervention in most instances. Psychotic symptoms in late-stage HIV infection are generally managed with atypical antipsychotic medications at the lowest effective dose, since standard neuroleptic medications have been associated with severe and difficult-to-treat extrapyramidal side effects [I]. Adjustment disorders may require treatment with psychotherapy or medication to prevent progression to a more severe psychiatric disturbance [III]. HIV-associated syndromes with psychiatric implications encompass wasting syndrome, fatigue, pain, and sexual dysfunction. Wasting syndrome has been effectively treated with testosterone (or its derivatives), growth hormone, and thalidomide [I]. Psychostimulants are one of the main interventions used for fatigue [II]. Chronic pain from peripheral neuropathy is often treated with tricyclic antidepressants and anticonvulsant medications, but published treatment studies of pain syndromes in patients with HIV infection have not supported their use [III]. In men, HIV-related hypogonadism can be treated with testosterone replacement [II].

Treatment of children and adolescents with antiretroviral medications has increased survival rates and slowed progression to AIDS [I]. The effectiveness of treatment of other mental and behavioral disorders associated with HIV infection in children and adolescents is largely unstudied.

\section{DISEASE DEFINITION, EPIDEMIOLOGY, AND NATURAL HISTORY}

\section{A. BASIC FACTS ABOUT HIV INFECTION, AIDS, AND TREATMENTS}

\section{Pathophysiology and virology}

Identified in 1984, HIV is a human retrovirus (Figure 1). A retrovirus contains RNA as its genetic material as well as the enzyme reverse transcriptase required to translate RNA into DNA within the human host cell. Once HIV RNA is transcribed into human DNA through the process of replication, it becomes a functional virus capable of producing profound immune deficiency, particularly cell-mediated immune dysfunction (Figure 2).

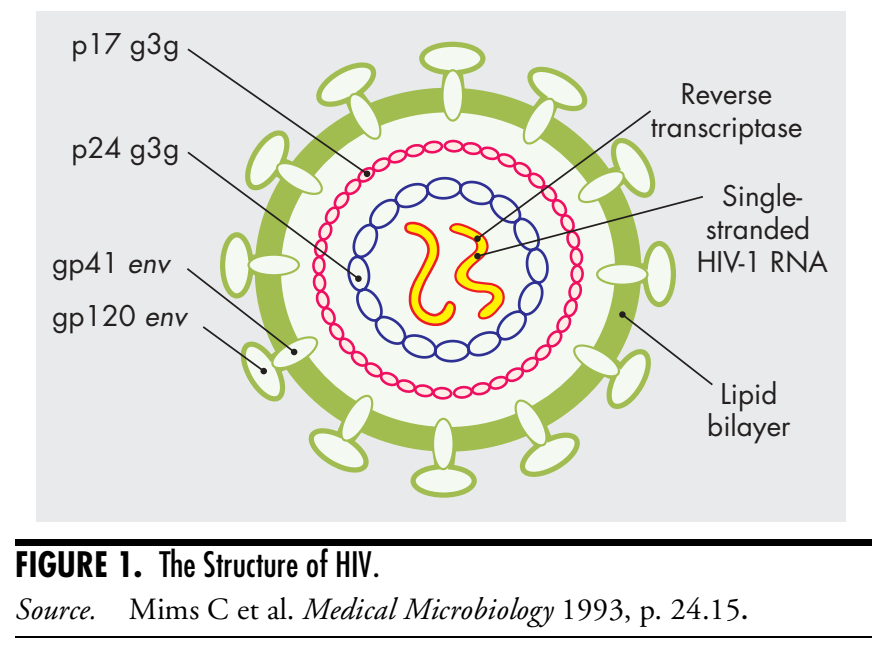




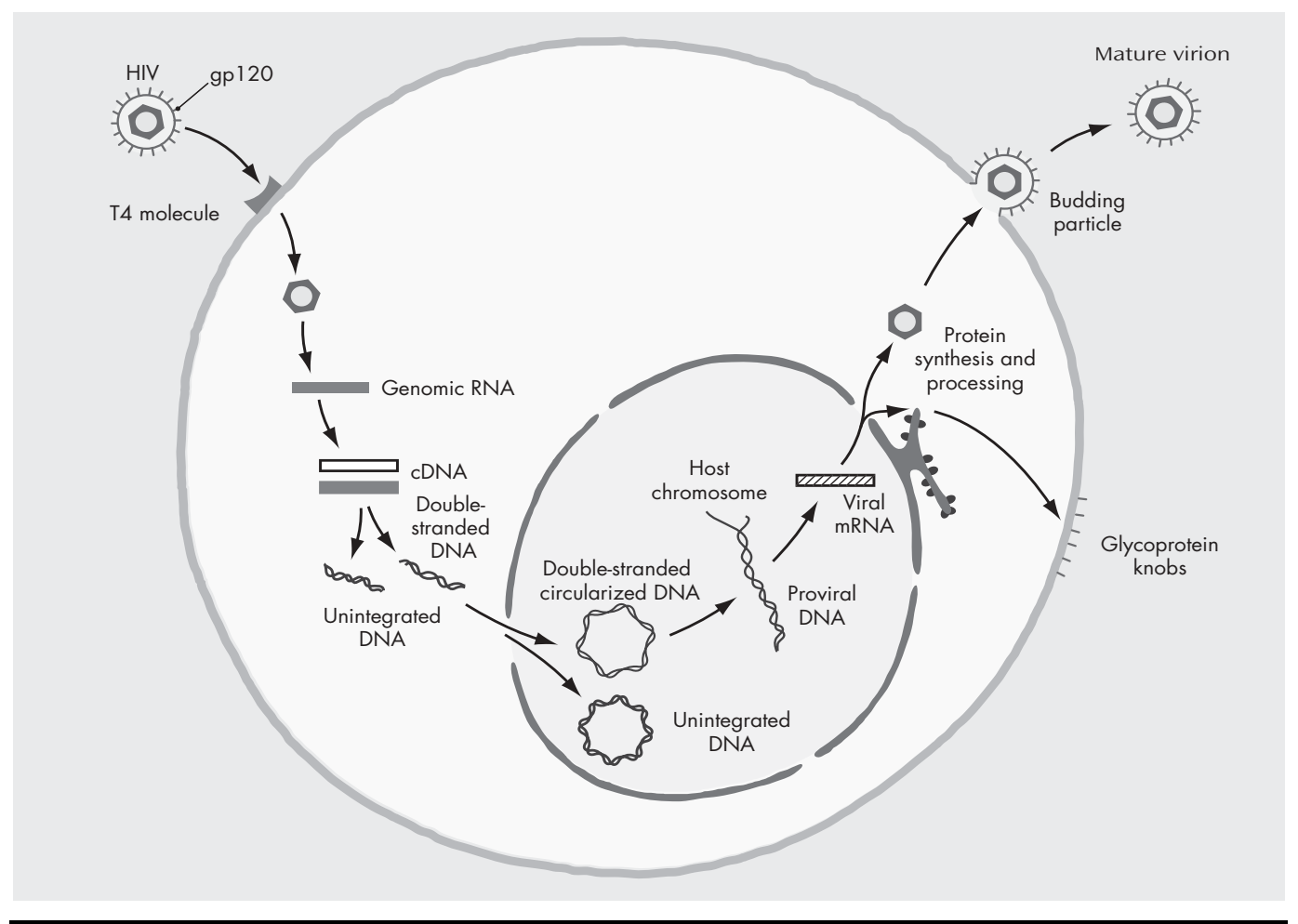

FIGURE 2. The HIV Replication Cycle.

Source. $\quad$ Mims C et al. Medical Microbiology 1993, p. 24.16.

As with other retroviruses, HIV has a rapid rate of genetic mutation. HIV-1 is the form of the virus that causes disease in most of the world, including the United States, Europe, Asia, Latin America, and most of Africa. HIV-2, discovered in 1986, causes a relatively small proportion of cases clustered in West Africa. Unless specified, the term HIV used in this guideline will refer to HIV-1.

HIV selectively infects certain cells within the human body, with the primary sites being blood mononuclear cells, particularly T-helper (CD4) lymphocytes and lymphoid tissues. HIV is neuropathic, invading the CNS early during the initial period of infection.

Counter to earlier beliefs, HIV does not become "dormant" but rather engages in an extraordinary battle with the immune system that begins and continues after initial infection. Once a host is infected with HIV, the virus begins a process of rapid replication, with billions of viral particles made soon after infection (Figure 3). At the same time, the host immune system mounts a response. The capacity of HIV to infect host cells and replicate, destroying CD4 cells in the process, is counteracted by the capacity of the host immune system to produce and maintain immune surveillance over the replicating virus. Current knowledge suggests that there is a viral "set point" that varies among individuals and constitutes a balance between viral reproduction and the immune response. This viral set point has prognostic significance (1). The growth of knowledge about the virology and pathophysiology of HIV has had enormous implications for treatment interventions.

\section{Epidemiology of HIV/AIDS}

HIV infection is a global pandemic, with cases of AIDS reported in nearly every country in the world. According to the Joint United Nations Programme on HIV/AIDS (UNAIDS), as of 
TABLE 1. U.S. Prevalence of HIV Infection, AIDS Cases, and Cumulative Deaths as a Result of AIDS Through December 1999

\begin{tabular}{ccc}
\hline $\begin{array}{c}\text { Persons living With } \\
\text { HIV Infection in 1999 }\end{array}$ & $\begin{array}{c}\text { Persons living } \\
\text { With AIDS in 1999 }\end{array}$ & $\begin{array}{c}\text { Cumulative Deaths } \\
\text { as a Result of AIDS }\end{array}$ \\
\hline 113,167 & 299,944 & 430,441 \\
\hline
\end{tabular}

Source. From the CDC HIV/AIDS Surveillance Report (3).

December 1999, more than 33 million people in the world are estimated to be living with HIV/AIDS, of which $95 \%$ live in the developing world. Globally, more than 16 million people have died of AIDS since the epidemic began (data available at www.unaids.org).

The Centers for Disease Control and Prevention (CDC) monitors the incidence, prevalence, morbidity, and mortality from HIV/AIDS in the United States (www.cdc.gov/nchstp/ hiv_aids/dhap.htm). Through December 1999, a cumulative total of more than 730,000 AIDS cases have been reported to the CDC; there have been 430,441 total deaths from AIDS in the United States (Table 1). Approximately 650,000-900,000 persons in the United States are currently infected with HIV, which is about $0.3 \%$ of the population or 1 in 300 Americans.

In recent years, there has been a remarkable change in the epidemiology of AIDS in the United States. The National Center for Health Statistics has reported that age-adjusted death rates from AIDS in the United States declined an unprecedented 47\% from 1996 to 1997, and AIDS fell from 8th to 14th among leading causes of death in the United States over the same time (2). For those aged 25-44, AIDS dropped from the leading cause of death in 1995 to the thirdleading in 1996 and the fifth-leading in 1997 (2). The drop in deaths and AIDS incidence has been attributed to the effectiveness of antiretroviral therapy, but the declines have been less apparent among women and minorities.

At the same time, a study by the CDC found that despite the decrease in AIDS deaths and incidence, the rate of new HIV infections has remained the same: about 40,000 new cases a year (3). Therefore, the overall prevalence of HIV infection in the United States has increased (4). Prevention of HIV infection remains a high priority.

Approximately $70 \%$ of new infections occur among men, of which $60 \%$ are men who have sex with men, $25 \%$ are injection drug users, and $15 \%$ are men exposed through heterosexual contact (3). Among women, $75 \%$ of new infections occur through heterosexual contact and $25 \%$ through injection drug use (5). African American and Hispanic communities are two distinct populations within which women, youth, and children are especially affected.

\section{Transmission of HIV}

HIV is transmitted through body fluids. It has been isolated from a variety of body fluids, including blood, semen, vaginal secretions, breast milk, urine, saliva, and tears. The risk of transmission through contact with a given fluid is related both to the amount of virus present in the fluid and to the type of exposure to it. HIV is found in such small concentrations in tears, saliva, and urine that transmission through casual contact with these fluids is theoretically possible but highly unlikely. On the other hand, behaviors that lead to certain types of exposure to blood, semen, vaginal secretions, and breast milk-all fluids with higher HIV concentrations-may lead to HIV transmission. HIV is spread primarily by unprotected sexual intercourse, irrespective of gender or sexual orientation, and sharing of unsterilized injection equipment for either medical or illicit purposes. It can be transmitted from an infected mother to an infant in utero during pregnancy, perinatally, or through breast-feeding.

\section{a) Sexual}

Sexual behaviors with exchange of body fluids can transmit HIV. While the rate of HIV transmission is somewhat higher for the recipient of semen than for the donating sexual partner, 
TABLE 2. Risk of HIV Transmission Associated With Various Sexual Activities

\begin{tabular}{|c|c|}
\hline Risk Level & Sexual Activity \\
\hline No risk & $\begin{array}{l}\text { Dry kissing } \\
\text { Body-to-body rubbing } \\
\text { Massage } \\
\text { Nipple stimulation } \\
\text { Using unshared inserted sexual devices } \\
\text { Being masturbated by partner without semen or vaginal fluids } \\
\text { Erotic bathing and showering } \\
\text { Contact with feces or urine on intact skin }\end{array}$ \\
\hline Theoretical risk & $\begin{array}{l}\text { Wet kissing } \\
\text { Cunnilingus with barrier } \\
\text { Anilingus } \\
\text { Digital-anal and digital-vaginal intercourse, with or without glove } \\
\text { Using shared but disinfected inserted sexual devices }\end{array}$ \\
\hline Low risk & $\begin{array}{l}\text { Sharing nondisinfected personal hygiene items (razors, toothbrushes) } \\
\text { Cunnilingus without barrier during or outside menstruation } \\
\text { Fellatio and ejaculation, with or without ingestion of semen } \\
\text { Fellatio, with or without condom } \\
\text { Penile-vaginal intercourse with condom } \\
\text { Penile-anal intercourse with condom }\end{array}$ \\
\hline High risk & $\begin{array}{l}\text { Penile-vaginal intercourse without condom } \\
\text { Penile-anal intercourse without condom } \\
\text { Coitus interruptus (intercourse with withdrawal before ejaculation) }\end{array}$ \\
\hline
\end{tabular}

Source. From Counselling Guidelines for HIV Testing (6).

transmission has been documented in both directions. Penile-anal and penile-vaginal intercourse are considered the highest risk behaviors, with transmission more likely in the presence of other sexually transmitted diseases or genital lesions or during sexual activities that cause a rupture of tissue or bleeding (Table 2).

\section{b) Injection drug use}

Sharing the equipment used to prepare and inject drugs with an HIV-infected person is a very efficient means of transmitting HIV and essentially amounts to a direct inoculation of viral particles from one person to another. The risk of transmission is directly related to the concentration of virus present in the blood and the volume of blood exchanged. Injection drug use is the second most common risk factor for HIV infection, and injection drug users account for an increasing proportion of AIDS cases ( $24 \%$ in 1997). It has been estimated that there are more than 1.5 million injection drug users in the United States (7).

\section{c) Blood transfusion}

Blood transfusion with infected blood products remains a significant risk for acquiring HIV in some parts of the world. In the United States, donated blood has been screened for antibodies to HIV-1 since 1985 and for antibodies to HIV-2 since 1992. Therefore, the risk of transmission from a blood transfusion has become extraordinarily low-less than $0.001 \%$. To further ensure that donated blood is not infected with HIV, since 1996 the American Red Cross has used the HIV antigen test. This test helps address the problem of false-negative HIV antibody tests in donors who may not have produced detectable antibodies after their initial infection. Before the use of lyophilized factor VIII, recurrent inoculation with pooled donated factor VIII was a major source of HIV transmission in hemophilia patients. 


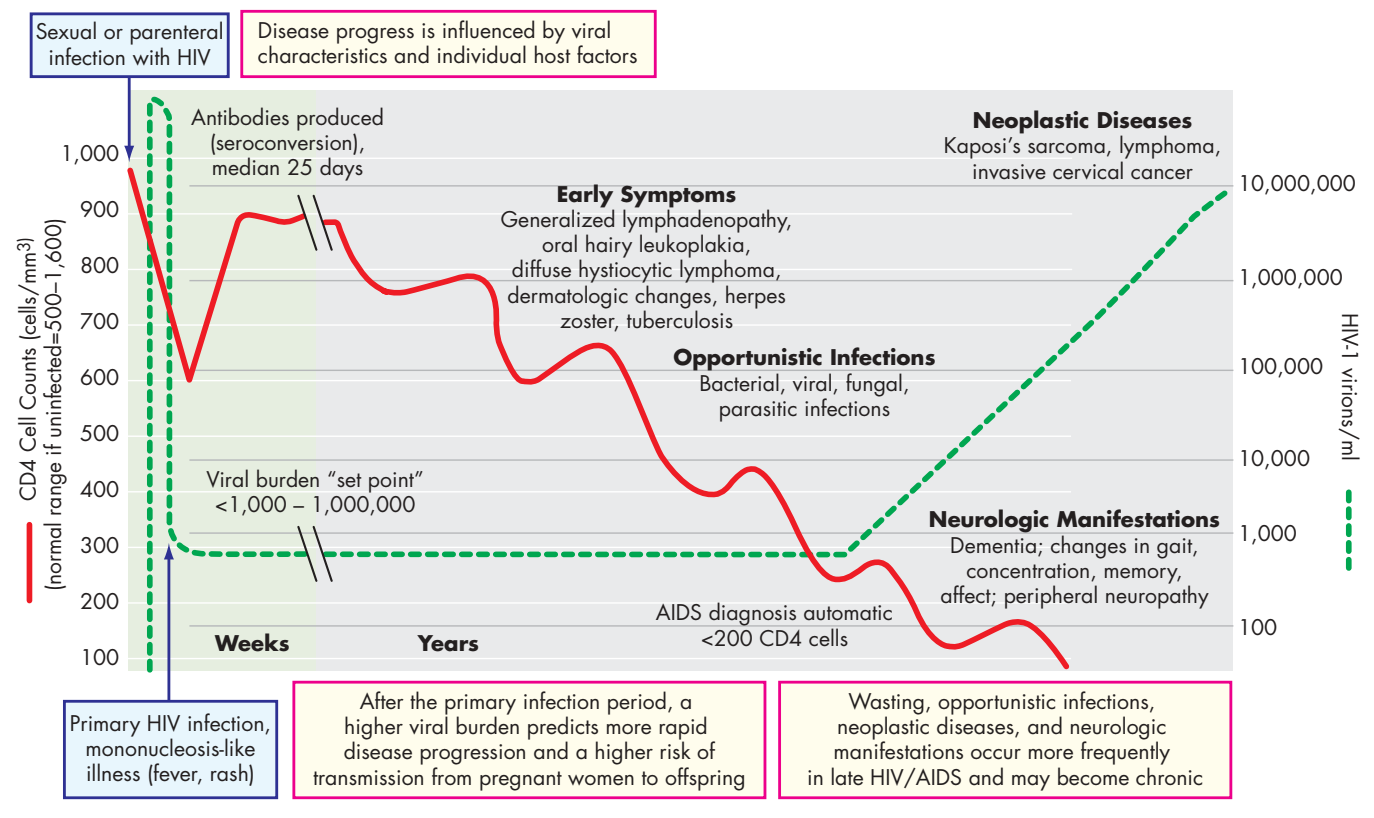

FIGURE 3. The Course of Untreated Adult HIV Infection.

Source. From the Southeast AIDS Training and Education Center, Emory University, Atlanta.

\section{d) Perinatal}

Infection from mother to infant can occur during gestation, delivery, or breast-feeding. Because breast milk contains significant numbers of lymphocytes that can lead to HIV transmission from mothers to newborns, it is recommended in the United States and other developed countries that HIV-infected mothers bottle-feed and not nurse their infants.

\section{e) Cofactors for transmission}

Cofactors can enhance but do not cause the transmission of HIV. Physical cofactors include the presence of sexually transmitted diseases (such as gonorrhea, syphilis, and chlamydia, which may cause genital lesions) or genital/mucous membrane bleeding during sexual activity. The use of mood or mind-altering substances may serve as a behavioral cofactor because they can lower sexual inhibitions, impair judgment, or increase impulsivity. Data are inconclusive regarding the effect of mind-altering substances on immunocompetence and HIV susceptibility or progression.

\section{Pathogenesis of HIV}

Three to 6 weeks after initial infection with HIV, there is a burst of viral replication, with wide dissemination of the virus throughout the body, particularly in lymphoid tissue and within the CNS. During this acute phase, approximately $50 \%-90 \%$ of people will experience a nonspecific "flu-like" syndrome of varying severity with fever, sore throat, rash, lymphadenopathy, and splenomegaly. Others do not have this seroconversion syndrome and may be unaware of their infection (8). As the host's immune system begins to recognize the pathogen and mounts a response to control the infection, plasma viral titers may drop 100-fold within 1 or 2 months of initial infection. When the host produces circulating antibodies against HIV, the host is said to "seroconvert," demonstrating a positive HIV antibody test. Although the immune system may 


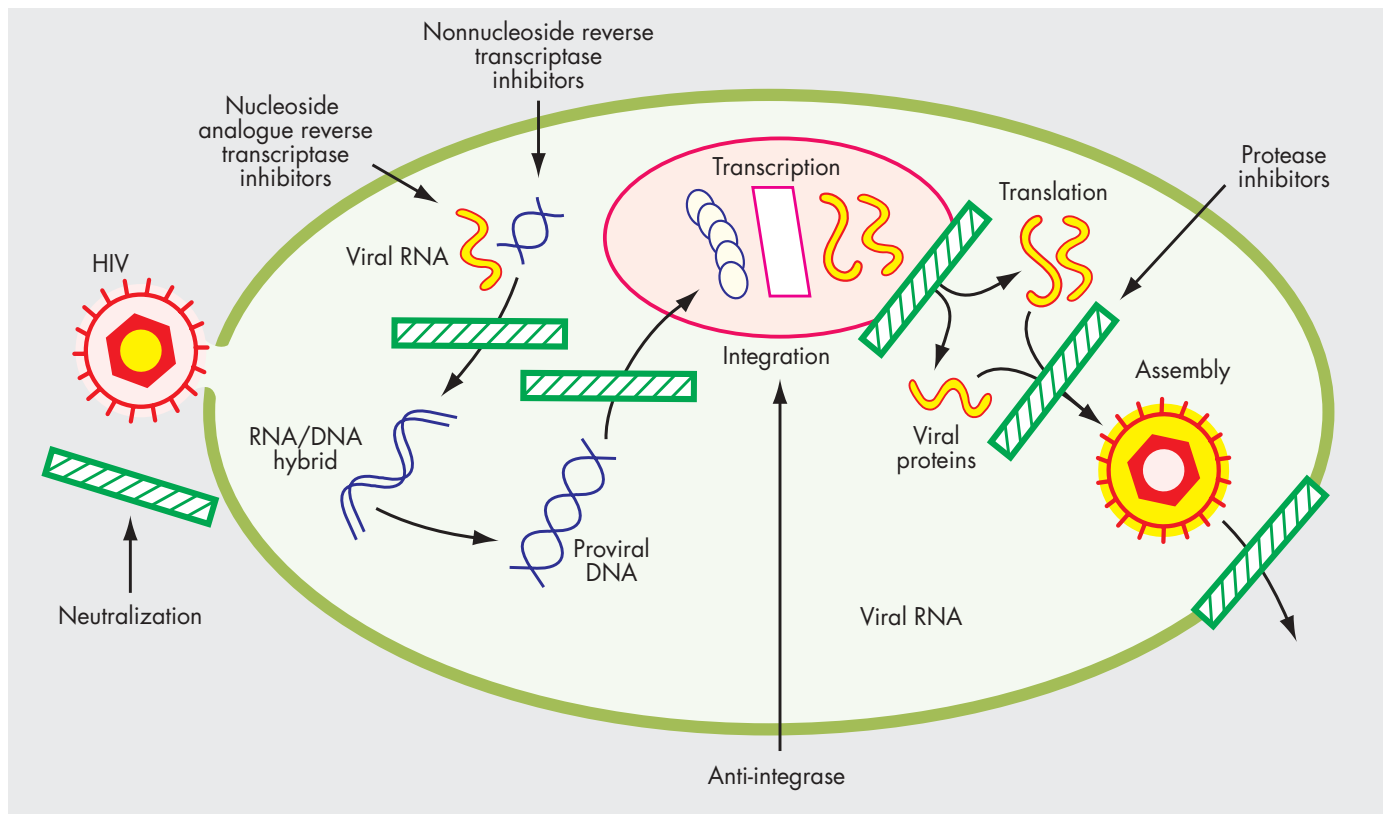

FIGURE 4. Antiviral Therapy Targets During the HIV Replication Cycle.

Source. Adapted from Paul (16).

appear to control the initial infection, chronic viral replication persists. There have been recent discoveries that "resting CD4 T cells" become infected rapidly after HIV transmission, and once HIV hides within resting T cells, antiretroviral therapy is unlikely to affect this sanctuary for $\operatorname{HIV}(9,10)$.

The acute phase is followed by a clinically asymptomatic phase, during which the body has established a dynamic balance between the capacity of the virus to replicate and infect new CD4 cells and the capacity of the body to produce more CD4 cells. Even so, as many as 10 billion viral particles are produced daily, with a plasma virus half-life of about 6 hours. The asymptomatic phase may last for many years until symptoms arise that indicate that HIV infection is progressing. Progression to a symptomatic phase of illness may occur more rapidly in those with immune systems that are compromised before HIV infection and in infants with undeveloped immune systems. Other factors influencing disease progression include mutations of cell surface receptors and host factors such as HIV-specific cytotoxic lymphocyte response (Figure 3).

Viral load, a measurement of how many viral particles are present in a cubic millimeter of blood, is determined by several different assays that can detect as few as 20 viral particles per cubic millimeter of blood. Below that threshold, viral load is reported as "nondetectable." This does not mean that there is no HIV in the blood. Furthermore, this method does not measure the amount of HIV in lymphoid tissue, semen, or CNS. While some studies have shown a small correlation between serum viral load and lymphoid tissue and CSF viral load, in later stages of HIV infection, plasma and CSF viral load are largely uncorrelated $(11,12)$. Thus, the CNS can be seen as an independent "reservoir" of HIV replication. Current practice is to use serum viral load measurements, in combination with assays of immune cells such as CD4 counts and genotypic testing for viral resistance, to guide clinical decisions about the initiation of or changes in antiretroviral medication (13-15). 
TABLE 3. 1993 Revised Classification System for HIV Infection and Expanded AIDS Surveillance Case Definition for AIDS Among Adolescents and Adults

\begin{tabular}{|c|c|c|c|}
\hline \multirow[b]{3}{*}{$\begin{array}{l}\text { T Cell Count } \\
\text { (cells/ } / \mu \text { l) }\end{array}$} & \multicolumn{3}{|c|}{ Clinical Categories } \\
\hline & A & B & C \\
\hline & $\begin{array}{l}\text { Acute (primary) HIV or persistent } \\
\text { generalized lymphadenopathy; } \\
\text { patient is asymptomatic }\end{array}$ & $\begin{array}{l}\text { Patient is symptomatic, } \\
\text { but condition does not meet } \\
\text { criteria for category A or C }\end{array}$ & $\begin{array}{l}\text { Patient has an AIDS } \\
\text { indicator condition a }\end{array}$ \\
\hline$\geq 500$ & A1 & B1 & $\mathrm{C} 1$ \\
\hline $200-499$ & A2 & B2 & $\mathrm{C} 2$ \\
\hline$<200^{\mathrm{a}}$ & A3 & B3 & C3 \\
\hline
\end{tabular}

Source. From the Centers for Disease Control and Prevention (17).

${ }^{a}$ As of Jan. 1, 1993, persons with AIDS indicator conditions (categories C1-C3) as well as those with T lymphocyte counts less than 200/ $\mu$ l (category A3 or B3) were categorized as AIDS cases in the United States and its territories.

New knowledge about the life cycle of HIV and the discovery of several classes of medications that disrupt the virus at different points in the replication cycle (Figure 4) benefit many patients who receive multidrug regimens, known as potent, combination, or highly active antiretroviral treatment. However, these regimens - which involve at least three medications, with short- and potential long-term side effects, taken multiple times per day for the life of the infected person-require strict adherence. The behavioral and psychological burden of early intervention is thus a mitigating factor against such an early and aggressive treatment approach. The rapid pace of changes to potent, combination, or highly active antiretroviral treatment guidelines adds to uncertainty about such interventions. Medications that can be taken less frequently are being developed to make the regimens more manageable.

\section{Assessment and staging of HIV disease}

HIV infection usually leads to clinical diseases that affect almost every organ system and present with a wide range of symptoms and syndromes. Accurate assessment and diagnosis of systemic as well as CNS impairment requires close medical and psychiatric management, with increasing vigilance as immune competence declines and the possibility of life-threatening disorders increases.

The CDC Revised Classification system for HIV infection and AIDS used CD4 counts and staging of illness (Table 3) to establish clinical categories for HIV disease. Generally, AIDS is defined by certain conditions marking advanced immunosuppression, such as a diagnosis of opportunistic infections (e.g., Pneumocystis carinii pneumonia) or other conditions (e.g., dementia or wasting). AIDS-defining criteria are listed in Table 4.

\section{Treatment for HIV infection}

Most of the world's HIV-infected population has little if any access to antimicrobial therapies for consequences of immune deficiency, let alone combination antiretroviral treatment. In the United States, revised standards of care for the treatment of HIV infection have improved treatment effectiveness, increased survival for patients, and potentially lessened development of viral resistance. Treatment is accomplished through numerous combinations of antiretroviral agents from four classes: nucleoside analogue reverse transcriptase inhibitors, nonnucleoside reverse transcriptase inhibitors, protease inhibitors, and the newest class, nucleotide analogues (Table 5). Guidelines for the use of antiretroviral agents in HIV-infected adults and adolescents are available from the HIV/AIDS Treatment Information Service (http://www.hivatis. org).

The current goal of antiretroviral therapy is to reduce viral load to undetectable levels and maintain such remission without interruption. Evidence suggests that the therapies suppress 
TABLE 4. AIDS-Defining Conditions That Emerge With Advancing Immunosuppression

\begin{tabular}{|c|c|}
\hline CD4 Cell Count (cells $/ \mathrm{mm}^{3}$ ) & Condition \\
\hline $200-500$ & $\begin{array}{l}\text { Thrush } \\
\text { Kaposi's sarcoma } \\
\text { Tuberculosis reactivation } \\
\text { Herpes zoster } \\
\text { Herpes simplex } \\
\text { Bacterial sinusitis/pneumonia }\end{array}$ \\
\hline $100-200$ & Pneumocystis carinii pneumonia \\
\hline $50-100$ & $\begin{array}{l}\text { Systemic fungal infections } \\
\text { Primary tuberculosis } \\
\text { Cryptosporidiosis } \\
\text { Cerebral toxoplasmosis } \\
\text { Progressive multifocal } \\
\text { leukoencephalopathy } \\
\text { Peripheral neuropathy } \\
\text { Cervical carcinoma }\end{array}$ \\
\hline $0-50$ & $\begin{array}{l}\text { Cytomegalovirus disease } \\
\text { Disseminated Mycobacterium } \\
\text { avium-intracellulare complex } \\
\text { Non-Hodgkin's lymphoma } \\
\text { Central nervous system lymphoma } \\
\text { HIV-associated dementia }\end{array}$ \\
\hline
\end{tabular}

replication but do not eradicate HIV from all parts of the body, particularly lymphoid tissue and the brain. Not all patients who initiate antiretroviral therapy respond. The lack of clinical response is likely explained by problems with adherence, suboptimal antiretroviral treatment potency, and genetic mutation of HIV strains (18). Many patients experience substantial side effects, and it is not uncommon for changes to be made in antiretroviral drug regimens because of such side effects. Adverse effects include lipodystrophy (fat redistribution syndromes), hyperlipidemia, nephrotoxicity, bone marrow suppression, neuropathy, and elevation of glucose to possibly diabetes mellitus-causing levels (19). Patients often experience nausea, diarrhea, sleep disturbances, and rashes. The cost of antiretroviral drugs alone is about $\$ 12,000$ per year for a single individual, and the long-term health consequences of these agents are not known.

Adherence is of utmost concern with antiretroviral treatment because the regimens are so unforgiving; even minor deviations from the prescribed regimen can result in viral resistance and permanent loss of efficacy for existing medications. Studies of antiretroviral treatment continue to indicate that near-perfect adherence is needed to adequately repress viral replication. The potential implications of nonadherence are troubling, as evidenced by a study that found a primary viral resistance rate to antiretrovirals of $16.3 \%$ among a cohort of recently infected individuals (20). This study documented what was earlier considered a theoretical concern-namely that persons newly infected with HIV may carry viral strains already resistant to available antiretroviral agents, indicating transmission of resistant viral strains from persons who were taking nonsuppressive multiple drug therapy or who were nonadherent to treatment (21).

As the U.S. HIV epidemic spreads increasingly among disadvantaged persons with limited resources who may have multiple comorbid disorders, significantly more psychosocial stressors, and less access to ongoing primary or mental health care, these individuals are at risk of not receiving the recommended treatment for HIV infection. Services for HIV patients must balance medical interventions with the emotional, economic, and social supports required for good quality of life and prevention of further transmission. 
TABLE 5. Antiretroviral Medications

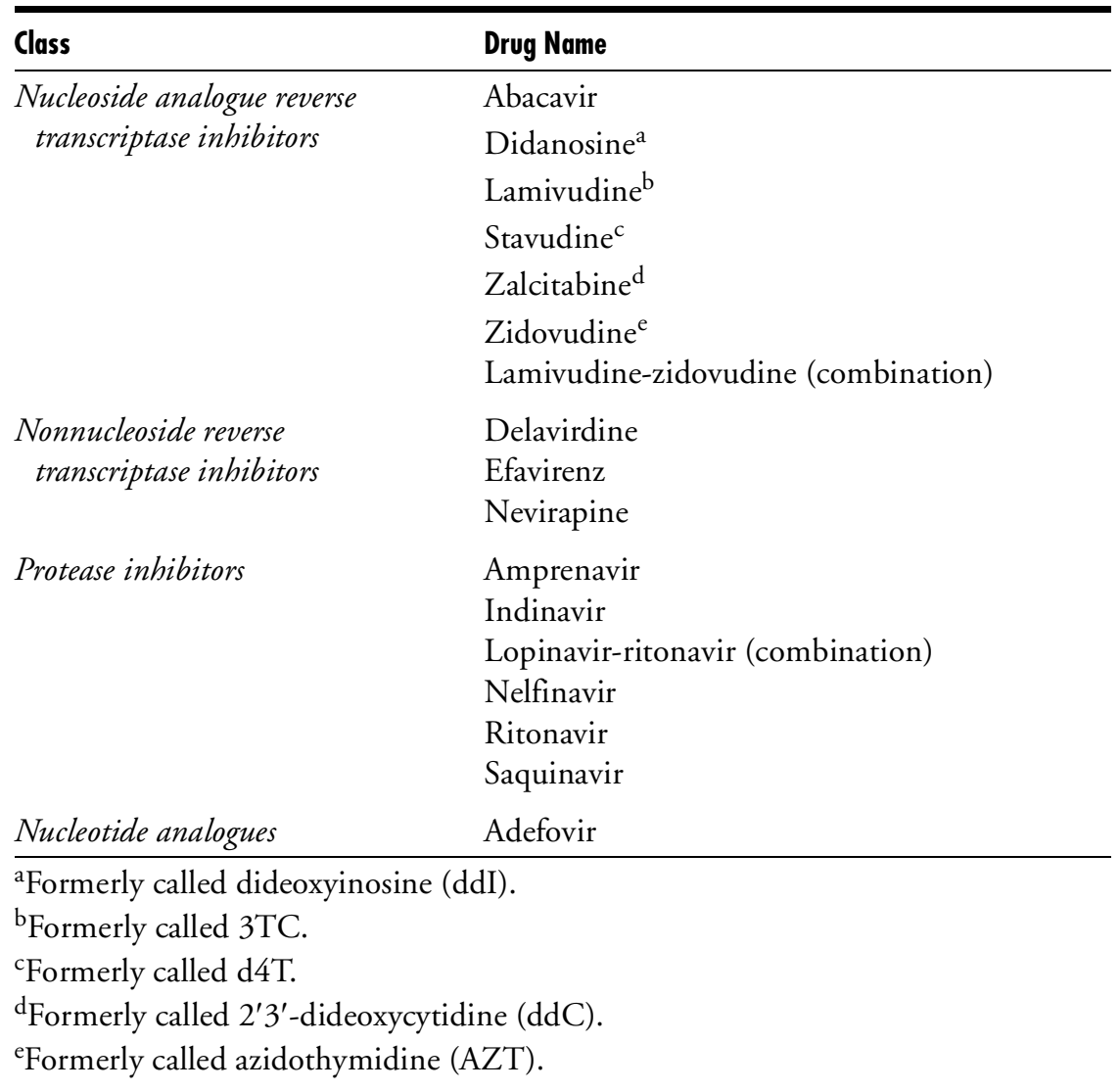

\section{B. ASSOCIATED FEATURES OF NEUROPSYCHIATRIC AND PSYCHOSOCIAL IMPORTANCE}

\section{Impact of HIV on the CNS}

Clinical evidence for direct infection of the CNS by HIV emerged in the mid-1980s, when patients began to survive their presenting opportunistic infections but went on to develop neuropsychiatric syndromes that could not be attributed to CNS opportunistic infections or neoplasms. Additional evidence included signs of neurocognitive impairment in adults, loss or arrest of developmental milestones in children, ability to culture HIV from CSF, neuropathological lesions of the brain at autopsy, and abnormalities observed through brain imaging techniques, including cerebral atrophy.

HIV invades the CNS early in the course of infection, entering by way of macrophages, which along with microglial cells are largely responsible for HIV replication within the CNS. While HIV does not infect neurons in the CNS, it causes neuronal death by other mechanisms. It is hypothesized that HIV infection of microglial cells in the CNS causes the elaboration of neurotoxins that, in turn, cause neuronal damage $(22,23)$. Neuronal dysfunction usually takes place at a slow rate, and it is not clear whether the immune system plays a role in keeping HIV in the CNS under control (24). Some researchers believe that high systemic viral load may "drive" neurological disease (25), but there is converging evidence that the CNS compartment differs from that of the peripheral bloodstream, with independent viral evolution/mutation (leading to viral resistance) and different viral load decay kinetics $(26,27)$. 
Major unanswered questions remain about the natural history of HIV in the CNS and whether current antiretroviral treatment regimens penetrate the blood-brain barrier sufficiently to reduce viral replication to the levels seen in the systemic circulation. Neuroprotective agents, immune modulators that increase macrophage activity within the CNS, and vaccines that boost immune response rather than prevent infection are all potential additional strategies to address neuropsychiatric syndromes.

\section{Clinical syndromes}

HIV infection can lead to neuropsychiatric syndromes that can occur at various stages of infection. Clinicians need to be aware of this possibility when evaluating new patients and observing changes in the patients whom they treat (28). Symptomatic HIV infection of the brain generally affects cognitive and motor functioning. Subtle neuropsychological impairment may be found in $22 \%-30 \%$ of otherwise asymptomatic patients with HIV infection $(29,30)$; these findings may or may not have functional significance. There is ample evidence for HIV causing the disorders HIV-associated dementia and HIV-associated minor cognitive motor disorder. HIV can affect other areas of the nervous system, causing syndromes such as painful sensory peripheral neuropathy or vacuolar myelopathy of the dorsolateral columns of the spinal cord. There are limited data in support of HIV infection of the CNS resulting in syndromes such as psychosis (31) or mania (32-34).

What separates HIV-associated dementia and HIV-associated minor cognitive motor disorder from the less severe cognitive changes seen in some HIV patients are the significance and duration of the functional deficits (Table 6, Table 7). HIV-associated minor cognitive motor disorder is a less severe disorder than HIV-associated dementia but does not necessarily progress to HIV-associated dementia (36). HIV-associated minor cognitive motor disorder is important to diagnose and treat, since it is thought to involve neuronal cell dysfunction rather than actual cell death, as seen in HIV-associated dementia. Both HIV-associated minor cognitive motor disorder and HIV-associated dementia are independent risk factors that decrease survival among patients with HIV infection $(37,38)$.

\section{HIV-associated dementia}

In contrast to Alzheimer's disease, which is a cortical dementia, HIV-associated dementia is classified as a subcortical dementia. HIV-associated dementia can produce different combinations of a clinical triad of progressive cognitive decline, motor dysfunction, and behavioral abnormalities. Symptoms commonly found in HIV-associated dementia include psychomotor slowing, decreased speed of information processing, impaired verbal memory and learning efficiency, and, later on, impairment in executive functioning. Behavioral manifestations vary throughout the range of HIV-related neurocognitive disorders, ranging from apathy to psychosis.

While the prevalence rate for HIV-associated dementia was estimated in the past to be $15 \%-20 \%$ of all AIDS patients (39), the incidence rate for HIV-associated dementia may be affected by the use of potent combination therapies. One report from the Multicenter AIDS Cohort Study (40) demonstrated declines of at least $50 \%$ in the incidence of HIV-associated dementia and CNS opportunistic complications (e.g., toxoplasmosis and primary CNS lymphoma) since the introduction of combination antiretroviral treatment in 1996. A second report has estimated that the incidence of HIV-associated dementia decreased from 21.1/1000 person-years in 1990-1992 to 14.7/1000 person-years in 1996-1997 (40). On the other hand, one group found that for patients who received combination antiretroviral treatment, the proportion of HIV-associated dementia as a percentage of all AIDS-defining illnesses rose from $4.4 \%$ to $6.5 \%$ between 1995 and 1997 (41). This shift in the rate of HIV-associated dementia is thought to reflect the decrease in rates of other AIDS-defining conditions, thereby leading to the relative rise in HIV-associated dementia cases. 
TABLE 6. Definitional Criteria for HIV-Associated Dementia

Criterion

1. Acquired abnormality in at least two of the following cognitive abilities

(present for $\geq 1$ month)

Attention/concentration

Speed of processing

Abstraction/reasoning

Visuospatial skills

Memory learning

Speech/language

2. At least one of the following:

Acquired abnormality in motor function or performance

Decline in motivation or emotional control or change in social behavior

3. Absence of clouding of consciousness during a period long enough to establish the presence of criterion 1

4. Exclusion of another etiology by history, physical, and psychiatric examination and appropriate laboratory and radiologic tests

\section{Description}

Cognitive decline verified by history and mental status examination. When possible, history should be obtained from an informant and examination should be supplemented by neuropsychological testing. The cognitive dysfunction must cause impairment of work or in activities of daily living, with impairment not attributable solely to severe systemic illness.

Abnormality verified by physical examination, neuropsychological tests, or both.

Change characterized by any of the following: apathy, inertia, irritability, emotional lability, or new-onset impaired judgment characterized by socially inappropriate behavior or disinhibition.

Source. From the American Academy of Neurology (35).

\section{TABLE 7. Definitional Criteria for HIV-Associated Minor Cognitive Motor Disorder}

\section{Probable Diagnosis (must meet all four criteria) ${ }^{a}$}

1. Acquired cognitive/motor/behavioral abnormalities, verified by both a reliable history and by neuropsychological tests

2. Mild impairment of work or activities of daily living

3. Does not meet criteria for HIV dementia or HIV myelopathy

4. No other etiology present

Source. From the American Academy of Neurology (35).

${ }^{a}$ A possible diagnosis of minor cognitive motor disorder can be given if criteria 1-3 are present and either 1) an alternative etiology is present and the cause of number 1 is not certain, or 2) the etiology of criterion 1 cannot be determined because of an incomplete evaluation.

\section{Other psychiatric syndromes}

Because HIV affects subcortical areas of the brain, it has been postulated that HIV may cause syndromes relating to mood and psychosis. New-onset psychosis and mania in HIV-infected patients tend to present at later stages of HIV disease and often occur in association with cognitive motor impairment. Lyketsos and colleagues (34) have described HIV-infected patients with so-called AIDS mania who present at advanced stages of HIV infection but generally have no family or personal history of mood disorder and have higher rates of comorbid dementia. 
TABLE 8. Etiologies of Delirium in HIV/AIDS Patients

\begin{tabular}{|c|c|}
\hline Intracranial & Extracranial \\
\hline Seizures & Medications and other drugs (not exhaustive) \\
\hline Infections & Amphotericin B \\
\hline Cryptococcal meningitis & Acyclovir \\
\hline Encephalitis due to HIV, herpes, cytomegalovirus & Ganciclovir \\
\hline Progressive multifocal leukoencephalopathy & Ethambutol \\
\hline Mass lesions & Trimethoprim/sulfamethoxazole \\
\hline Lymphoma & Pentamidine \\
\hline \multirow[t]{21}{*}{ Toxoplasmosis } & Foscarnet \\
\hline & Ketoconazole \\
\hline & Sedative/hypnotics \\
\hline & Cycloserine \\
\hline & Opiate analgesics \\
\hline & Isoniazid \\
\hline & Rifampin \\
\hline & Zidovudine or didanosine \\
\hline & Vincristine \\
\hline & Dapsone \\
\hline & Drug or alcohol withdrawal \\
\hline & Infection/sepsis \\
\hline & $\begin{array}{l}\text { Endocrine dysfunction/metabolic } \\
\text { abnormality }\end{array}$ \\
\hline & $\begin{array}{l}\text { Hypoglycemia due to pentamidine, } \\
\text { protease inhibitors }\end{array}$ \\
\hline & Hypoxia due to pneumonia \\
\hline & Nonendocrine organ dysfunction \\
\hline & $\begin{array}{l}\text { Renal failure due to HIV nephropathy or } \\
\text { medication toxicity }\end{array}$ \\
\hline & $\begin{array}{l}\text { Liver failure due to comorbid hepatitis and } \\
\text { medication toxicity }\end{array}$ \\
\hline & Nutritional deficiencies \\
\hline & Wasting syndrome \\
\hline & $\begin{array}{l}\text { Failure to replace trace elements or vitamins } \\
\text { in total parenteral nutrition }\end{array}$ \\
\hline
\end{tabular}

Source. Adapted from Bialer et al. (50).

They hypothesized that mania in this context is directly caused by HIV brain infection. Mijch and colleagues (32) conducted a prospective case-control study to test the hypothesis put forward by Lyketsos et al. In their study of 19 HIV patients with secondary mania, they found that treatment with zidovudine, which is known to penetrate the blood-brain barrier well, provided a protective effect against the development of mania. In addition, they found the incidence rate of HIV-associated dementia in the subjects with mania to be significantly greater than in HIV subjects without mania.

New-onset psychotic symptoms have been reported in HIV-positive individuals in the absence of medical/iatrogenic causes or concurrent substance abuse $(42,43)$. One case-control study systematically examined patients with HIV infection that predated the onset of psychosis (31). In this study, the psychotic subjects had significantly higher rates of past stimulant and sedative-hypnotic abuse and higher mortality at follow-up. While the study was limited by a sample size of 20, the authors postulated that direct effects of HIV infection on the brain could be one possible cause of new-onset psychosis in their subjects. 
TABLE 9. Central Nervous System Manifestations of HIV-1 Infection

\begin{tabular}{ll}
\hline Type of Manifestation & Condition \\
\hline Acute HIV-1 infection & Viral meningitis \\
& Encephalitis \\
& Ascending polyneuropathy \\
Opportunistic infections & Toxoplasma cerebritis \\
(late HIV-1 infection) & Cryptococcal meningitis \\
& Progressive multifocal leukoencephalopathy \\
& Neurosyphilis \\
& Mycobacterium tuberculosis meningitis \\
& Cytomegalovirus encephalitis \\
& Herpes simplex encephalitis \\
Neoplastic disease (late HIV-1 infection) & CNS lymphoma \\
& Kaposi's sarcoma \\
Other manifestations & HIV-associated dementia \\
& HIV-associated minor cognitive motor disorder \\
\hline
\end{tabular}

\section{Differential diagnosis of neuropsychiatric syndromes}

Differential diagnosis of an acute change in mental status includes but is not limited to delirium. Delirium occurs frequently in the medically ill and is more likely when a patient's illness is more severe. Estimates of rates of delirium in HIV patients range from $43 \%$ to greater than $65 \%$ in late-stage AIDS (44-46). Definition and management of the syndrome of delirium is well described in the APA Practice Guideline for the Treatment of Patients With Delirium (47). Identification of the delirious state and intervention to correct underlying causes reduces morbidity and mortality. In persons with HIV infection, the most common causes are iatrogenic and psychoactive-substance-induced toxicity, infection, neoplasms, and metabolic disturbances. Some antiretroviral medications can cause delirium (e.g., zidovudine at high doses [48] or efavirenz [49]) (Table 8).

The term "HIV encephalopathy" is occasionally used to refer to acute-onset cognitive deficits in adults, but its definition and usage vary. A wide range of conditions that primarily involve the CNS needs to be considered within the psychiatric differential diagnosis, since these conditions may present with psychiatric symptoms or cause psychiatric syndromes including psychosis (with or without an affective component) and mood episodes (mania, hypomania, depression) (51) (Table 9). The same concerns exist for medications commonly used to treat conditions associated with HIV infection (Table 10).

In adults and children with HIV infection, changes in mental status or the emergence of new psychiatric or cognitive disorders requires ruling out treatable and reversible causes; medical causes are of increasing concern if CD 4 counts are low or viral load has begun to rise. Psychiatrists should consider adding assessments of basic immune function and viral load, CSF examination, and brain imaging studies to the standard medical workup of new- or acute-onset psychiatric syndromes for patients with or at high risk for HIV infection (Table 11).

\section{Pediatric HIV/AIDS syndromes}

To date, the proportion of children born with HIV who survive until the age of 9 is approximately $50 \%$, but antiretroviral interventions will increase the number of infected youth who enter adolescence. HIV-related symptoms may develop at any age, but a prospective collaborative study found that only $10 \%$ of children were symptomatic before the onset of an AIDS-defining illness (53).

Certain clinical presentations characterized by lymphadenopathy, parotitis, skin diseases, and recurrent respiratory tract infections are associated with longer survival, while lymphoid 
TABLE 10. Neuropsychiatric Side Effects of Selected Medications Used in HIV Disease

\begin{tabular}{|c|c|c|}
\hline Drug & Target Illness & Side Effects \\
\hline Acyclovir & Herpes encephalitis & $\begin{array}{l}\text { Visual hallucinations, depersonalization, tearfulness, } \\
\text { confusion, hyperesthesia, hyperacusis, thought } \\
\text { insertion, insomnia }\end{array}$ \\
\hline Amphotericin B & Cryptococcosis & Delirium, peripheral neuropathy, diplopia \\
\hline $\begin{array}{l}\beta \text {-Lactam } \\
\text { antibiotics }\end{array}$ & Infections & Confusion, paranoia, hallucinations, mania, coma \\
\hline Co-trimoxazole & $\begin{array}{l}\text { Pneumocystis carinii } \\
\text { pneumonia }\end{array}$ & Depression, loss of appetite, insomnia, apathy \\
\hline Cycloserine & Tuberculosis & $\begin{array}{l}\text { Psychosis, somnolence, depression, confusion, tremor, } \\
\text { vertigo, paresis, seizure, dysarthria }\end{array}$ \\
\hline Didanosine & HIV & $\begin{array}{l}\text { Nervousness, anxiety, confusion, seizures, insomnia, } \\
\text { peripheral neuropathy }\end{array}$ \\
\hline Efavirenz & HIV & Nightmares, depression, confusion \\
\hline Foscarnet & Cytomegalovirus & $\begin{array}{l}\text { Paresthesias, seizures, headache, irritability, } \\
\text { hallucinations, confusion }\end{array}$ \\
\hline Interferon- $\alpha$ & Kaposi's sarcoma & Depression, weakness, headache, myalgias, confusion \\
\hline Isoniazid & Tuberculosis & $\begin{array}{l}\text { Depression, agitation, hallucinations, paranoia, } \\
\text { impaired memory, anxiety }\end{array}$ \\
\hline Lamivudine & HIV & Insomnia, mania \\
\hline Methotrexate & Lymphoma & Encephalopathy (at high dose) \\
\hline Pentamidine & $\begin{array}{l}\text { Pneumocystis carinii } \\
\text { pneumonia }\end{array}$ & Confusion, anxiety, lability, hallucinations \\
\hline Procarbazine & Lymphoma & $\begin{array}{l}\text { Mania, loss of appetite, insomnia, nightmares, } \\
\text { confusion, malaise }\end{array}$ \\
\hline Quinolones & Infection & $\begin{array}{l}\text { Psychosis, delirium, seizures, anxiety, insomnia, } \\
\text { depression }\end{array}$ \\
\hline Stavudine & HIV & $\begin{array}{l}\text { Headache, asthenia, malaise, confusion, depression, } \\
\text { seizures, excitability, anxiety, mania, early morning } \\
\text { awakening, insomnia }\end{array}$ \\
\hline Sulfonamides & Infection & $\begin{array}{l}\text { Psychosis, delirium, confusion, depression, } \\
\text { hallucinations }\end{array}$ \\
\hline Thiabendazole & Strongyloidiasis & Hallucinations, olfactory disturbance \\
\hline Vinblastine & Kaposi's sarcoma & Depression, loss of appetite, headache \\
\hline Vincristine & Kaposi's sarcoma & Hallucinations, headache, ataxia, sensory loss \\
\hline Zalcitabine & HIV & $\begin{array}{l}\text { Headaches, confusion, impaired concentration, } \\
\text { somnolence, asthenia, depression, seizures, peripheral } \\
\text { neuropathy }\end{array}$ \\
\hline Zidovudine & HIV & $\begin{array}{l}\text { Headache, malaise, asthenia, insomnia, unusually vivid } \\
\text { dreams, restlessness, severe agitation, mania, auditory } \\
\text { hallucinations, confusion }\end{array}$ \\
\hline
\end{tabular}

Source. Adapted from Grant and Atkinson (52).

interstitial pneumonitis and thrombocytopenia form an intermediate survival group. The worst prognosis is associated with bacterial infections, progressive neurological disease, anemia, and fevers (54).

HIV infection that occurs at the time of birth rather than early in gestation is thought to be associated with a better prognosis. While at any given time many HIV-infected children may not experience significant HIV-related medical morbidity, their overall health outcome may be compromised by the conditions of poverty. 
TABLE 11. Evaluation of Altered Mental Status in Patients With HIV/AIDS

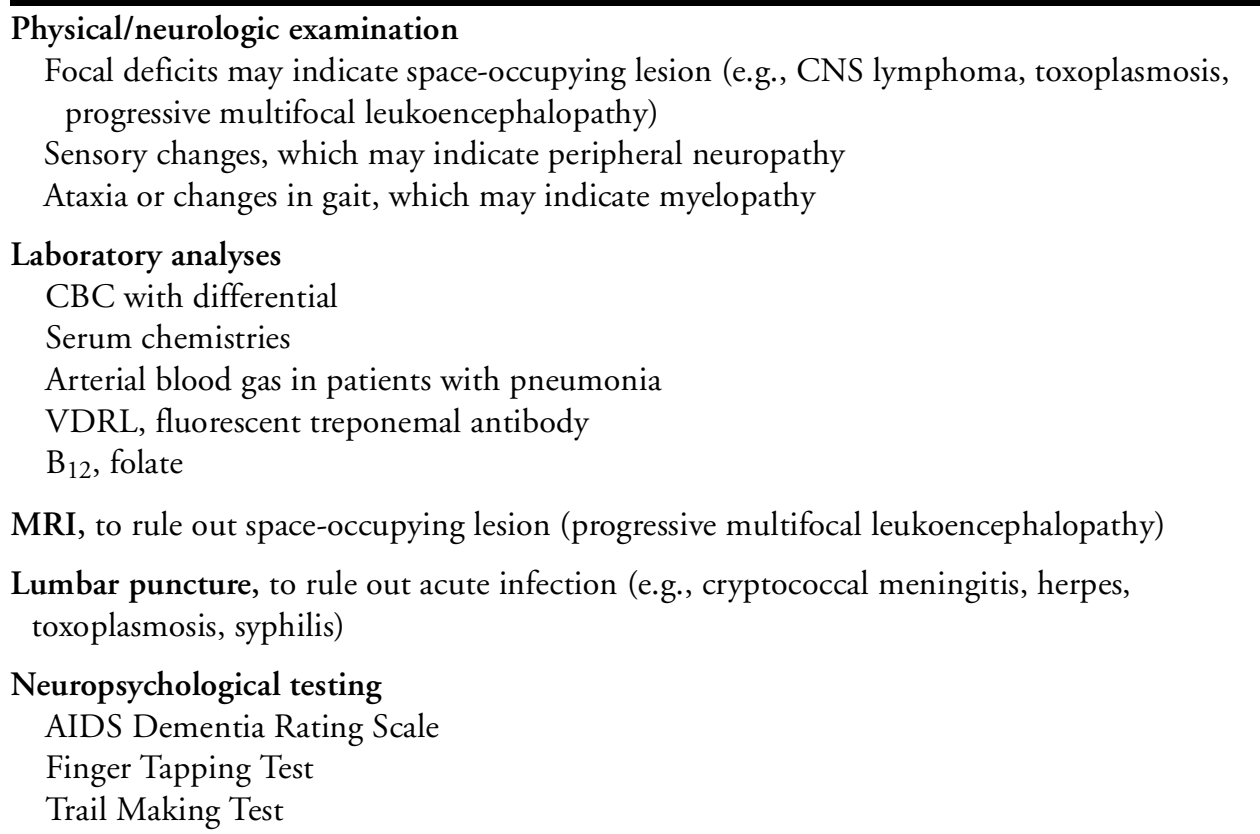

Source. Adapted from Bialer et al. (50).

The impact of HIV on the developing nervous system of children is more significant than on the developed adult nervous system, and certain cognitive deficits may occur in some infected youth. The term "dementia" is not commonly used to describe cognitive deficits in children by pediatricians or child psychiatrists, who refer to this condition as either HIV-associated progressive encephalopathy or HIV encephalopathy. Nonetheless, "dementia due to HIV disease" in children is specifically mentioned in DSM-IV (p. 148).

HIV-associated progressive encephalopathy in children is characterized by a triad of symptoms: impaired brain growth, progressive motor dysfunction, and loss or plateau of developmental milestones (55). Progressive encephalopathy needs to be distinguished from mental retardation secondary to other causes, such as maternal drug addiction and prematurity, which can be determined only by longitudinal assessment. Despite the general fact that progressive encephalopathy is observed in the context of immunosuppression, markers of immunologic functioning (e.g., CD4 count) do not correlate with degree of neurocognitive impairment (54). The prevalence rate of progressive encephalopathy in a cohort of $128 \mathrm{HIV}$-infected children was $21 \%$ during a mean follow-up period of 24 months. Progressive encephalopathy was the first AIDSdefining condition in $67 \%$ of the group, and mean survival after diagnosis was 14 months (56).

Although many children with HIV are considered to be asymptomatic, numerous studies document at least some cognitive and language delays (57). In a sample of $36 \mathrm{HIV}$-infected children who were less than 10 years old, brain abnormalities detected by means of computerized tomography scans were significantly correlated with receptive and expressive language deficits, with expressive language more severely impaired than receptive language among those children with progressive encephalopathy (58). In addition, visual motor deficits are common and may be correlated with disease progression (59).

\section{PREVALENCE OF COMORBID PSYCHIATRIC CONDITIONS}

Since early in the HIV epidemic, researchers have studied the prevalence of psychiatric disorders in persons with HIV infection (60-63). Often these psychiatric disorders predated HIV 
infection or occurred during the course of living with the disease; they are not necessarily attributable to neuropathic effects of HIV. Psychiatric illness is generally related to poorer functioning and quality of life as well as increased use of HIV-related hospital services (64). A study conducted by researchers in California found that depression was associated with shorter survival times in men with HIV infection (65).

Prevalence rates of psychiatric disorders reported in published studies vary widely. In general, higher rates of psychiatric disorders are seen in later stages of HIV infection and are reported in studies that assess patients receiving care in HIV medical or psychiatric clinics rather than community-based samples (66). On one extreme, recent data from the national, multisite HIV/ AIDS Mental Health Services Demonstration Program revealed high rates of depression $(60 \%)$, dysthymic disorder (25\%), and anxiety disorders (25\%) among persons seeking HIVrelated mental health services in the public sector (67). The study found high rates of comorbid substance use disorders, with nearly $50 \%$ of all patients also having a diagnosis of alcohol or drug dependence. These higher rates are most likely linked to characteristics of the population studied: HIV-positive individuals voluntarily seeking psychiatric care, a population that may be more reflective of patients receiving care from community mental health clinics. In contrast, prevalence studies in selected community-based samples have found much lower rates of psychiatric disorders, such as rates of major depressive disorder in the $4 \%-14 \%$ range (68-73).

\section{FORMULATION AND IMPLEMENTATION OF A TREATMENT PLAN}

\section{A. INDIVIDUALS AT HIGH RISK FOR HIV INFECTION}

HIV prevention strategies are an essential component of the comprehensive treatment of specific psychiatric populations and for other psychiatric patients who manifest high-risk sexual and drug use behavior. Some psychiatric patients, such as those confined to forensic units, in long-term hospitals, and locked nursing homes have almost no access to preventive strategies other than those provided and supported by staff. Administrators and institutions should formulate policies that support the full range of HIV prevention steps outlined in this section. Primary prevention strategies are those that seek to avert initial infection. Secondary and tertiary prevention strategies target infected individuals in order to prevent further transmission and reduce HIV-related medical complications, respectively.

\section{Psychiatric management}

Optimum management of patients at high risk for HIV infection involves a wide range of psychiatric skills: comprehensive diagnostic evaluations, assessment of possible medical causes of new-onset symptoms, initiation of specific treatment interventions, and a keen understanding of psychodynamic issues. In some institutional settings, psychiatrists serve as primary clinicians of both medical and psychiatric care. In such situations, psychiatrists should be mindful to include HIV risk assessment and prevention as part of patients' treatment plans.

\section{a) Obtaining a risk history}

A psychiatrist will not be aware of a patient's risk for HIV infection unless risk behavior is accurately assessed. Such an assessment should be considered in every psychiatric evaluation in order to identify individuals who are at high risk due to specific behaviors. Factors such as acute episodes of psychiatric illness, stressful or traumatic life events, and the developmental stage of the patient (e.g., initiation of sexual activity in adolescents) contribute to the need for ongoing appraisal of patient risk. Psychiatrists are particularly well-placed to assess HIV risk because 
TABLE 12. Items for Clinicians to Cover When Conducting an Assessment of HIV Risk Behavior

Frequency of sexual intercourse (vaginal, anal, oral)

Number, gender, and known HIV risk of sex partners

Whether the patient has traded sex (for money, drugs, a place to stay, cigarettes)

Past and current symptoms of sexually transmitted infections

Use of condoms and other contraceptive methods

Use of drugs, particularly those that are injected or sniffed

Sharing of needles, syringes, or other injection equipment

Source. From McKinnon et al. (74).

they often follow patients for lengthy periods of time, which allows for multiple opportunities to assess behavior (74).

At times, the clinical state of the patient may preclude an accurate assessment, such as when the patient is acutely psychotic or intoxicated. In this situation, the risk history may need to be obtained either when the patient is able to provide valid answers or with the assistance of family or friends. Psychiatrists should be knowledgeable about which specific sexual behaviors are more likely to result in HIV transmission (Table 2). When conducting an assessment of risk behavior, psychiatrists should convey a nonjudgmental attitude.

When carrying out a risk assessment, it is important to clarify the vocabulary and cultural beliefs of the patient. For instance, it is not uncommon for patients and clinicians to use different terms to describe sexual or drug use behaviors, and slang terms change quickly. The $\mathrm{Na}-$ tional Institute on Drug Abuse has published a community drug alert that outlines common street drugs and their slang names, which is available at www.drugabuse.gov. Clinicians may be able to clarify risk behavior terms by first describing a risk behavior and then asking a patient what he or she would call that behavior (Table 12).

\section{b) HIV antibody testing}

Attitudes about HIV antibody testing have changed with the development of HIV treatment interventions and educational efforts. Formerly, patients were unwilling to learn their HIV status, since knowing could cause emotional distress, could engender possible discrimination, and did not lead to better clinical outcomes. Public policy now promotes earlier identification of HIV infection so that newly infected persons can be medically monitored and receive antiretroviral treatment as appropriate. Yet HIV testing still carries risks due to worries and fears associated with HIV/AIDS, as well as the possibility of physical assault by a partner or other relation after HIV diagnosis (75). Disclosure of HIV status to family, friends, or employers can be quite problematic for some patients.

Discussion of the pros and cons of a routine baseline HIV test are part of a comprehensive approach to HIV prevention in high-risk patients. Psychiatric units or individual practitioners who conduct HIV testing should be aware of their obligation to provide the necessary pre- and posttest counseling (76). Elements of pre- and posttest counseling include an explanation of the HIV test, including risks and benefits, confidentiality of the results, discussion of risk behavior and risk reduction strategies, and plans for dealing with a positive or negative test result. Federal guidelines for counseling have been established by the CDC (http://www.cdc.gov/hiv/ pubs/hivctsrg.pdf).

Clinicians should remember that the timing of when to undergo HIV testing is a distinct clinical decision. For instance, it is generally not advisable to test a patient for HIV while he or she is confused or intoxicated with alcohol or drugs. In the event that a patient cannot give informed consent, the psychiatrist should be familiar with the local legal requirements regarding HIV testing and disclosure (77). Anonymous and confidential HIV testing is often provided by state public health agencies or community-based organizations; some patients prefer ano- 
nymity and may be more likely to agree to testing given this option. Many state health departments conduct "partner notification" or prevention counseling and referral services to identify and test past or present sexual partners or drug injection equipment-sharing partners of a newly reported HIV-seropositive person.

The American College of Obstetricians and Gynecologists now recommends that an HIV antibody test be offered during annual exams to all women seeking preconception care (not just pregnant women), which reflects the growing awareness that many women may not properly assess their personal risk for HIV infection (78). This recommendation goes beyond the previous recommendation that pregnant women undergo HIV antibody testing so that HIV-infected women can consider treatment with antiretroviral medication. For female patients who are at risk for HIV infection and for pregnancy, conducting a baseline pregnancy test along with a baseline HIV test is advised.

\section{c) Risk reduction strategies}

Risk reduction strategies include education of patients about behaviors that place them at risk for HIV infection, active discussions of changes in behavior, and treatment of problems that promote risky behavior. It is important to view undergoing an HIV antibody test as a "teachable moment," when counseling about risk for HIV can be tailored to the specific behavior of the patient and an individualized risk reduction plan can be developed.

Because successful risk reduction requires more than knowledge of risk, ongoing discussions between patient and psychiatrist can help provide the motivating and skill-building factors that help ensure consistent changes in behavior. Psychiatrists should consider unconscious motivations that may contribute to risk-taking behavior when developing risk reduction strategies. Some patients may need to be referred to community-based organizations or other clinicians who offer specific risk reduction programs (e.g., needle exchange programs or skills training groups). For some patients, risk reduction strategies can include extended counseling and case management, such as that modeled by the CDC (79).

When appropriate, psychiatrists should determine whether patients have access to condoms and the skills to use them (Table 13). Skills to discuss and negotiate safer sex with partners may need to be developed; psychotherapy can provide an opportunity to practice communication skills through role playing. Clinicians should be alert to feelings of powerlessness in sexual situations for patients with histories of sexual abuse and to the real possibility of violence for some if a sexual partner is threatened or angered.

Psychiatric conditions that could theoretically increase patient risk for engaging in high-risk behavior include impulse control disorders, untreated depression, hypersexuality associated with mania, psychotic disorders, mental disorders due to a general medical condition, binge alcohol or drug use, and personality disorders.

\section{d) Postexposure prophylaxis}

The premise underlying postexposure prophylaxis is that chemoprophylaxis during a window of opportunity may prevent initial cellular infection and local propagation of HIV, thus allowing the host immune defenses to eliminate the inoculum of virus (81). Currently, postexposure prophylaxis is recommended for known occupational exposure, especially percutaneous or mucous membrane exposure, to blood or other body fluids.

Psychiatrists who serve as administrators of mental health facilities should formulate policies and protocols for the expedient treatment of health care professionals or patients who have had such exposure. The protocol requires a rapid assessment of risk and, where risk is present, beginning a multiple drug regimen as soon as 1-2 hours after exposure and not later than 24-36 hours. It further requires 4 weeks of treatment with two or three antiretroviral agents that can have significant side effects. 
Condoms must be used consistently and correctly to provide maximum protection. Consistent use means using a new condom with each act of intercourse. Latex condoms and polyurethane condoms provide protection; lambskin condoms should not be used. Correct condom use includes all of the following steps.

- Check the expiration date.

- Use a new condom for each act of vaginal, anal, or oral intercourse.

- Put on the condom as soon as erection occurs and before any vaginal, anal, or oral contact with the penis.

- Apply lubricant to penis before and after putting the condom on. This will provide greater satisfaction for both the insertive and the receptive partners.

- Hold the tip of the condom and unroll it onto the erect penis, leaving space at the tip of the condom, yet ensuring that no air is trapped in the tip of the condom.

- Adequate lubrication is important to prevent condom breakage, but use only water-based lubricants, such as glycerine or lubricating jellies (which can be purchased at any pharmacy). Oil-based lubricants, such as petroleum jelly, cold cream, hand lotion, or baby oil, can weaken the condom.

- Withdraw from the partner immediately after ejaculation, holding the condom firmly to the base of the penis to keep it from slipping off.

Source. From the CDC National Center for HIV, STD and TB Prevention, Division of HIV/AIDS Prevention (80).

The CDC has issued guidelines for the use of antiretroviral medication following health care worker occupational exposure to HIV (82). There is a National Clinicians' Postexposure Hotline (888-448-4911) that can be accessed 24 hours a day for guidance in cases of possible or known exposure to HIV. A web site (www.ucsf.edu/hivcntr) offers a wealth of information to address this issue.

Data are being gathered to evaluate the use of postexposure prophylaxis in other exposure situations, but it is being increasingly offered for known or possible sexual exposure to HIV. Public health messages should emphasize that postexposure prophylaxis should be used only when primary prevention methods such as use of condoms or avoidance of high-risk behaviors have failed. Clinicians should counsel patients who receive postexposure prophylaxis to reduce their chance of future exposure (81).

\section{Specific treatment situations}

\section{a) Patients with substance use disorders}

The best way to prevent the spread of HIV through injection drug use is effective primary prevention of drug use. For people who are already injecting opioids, eliminating this behavior through adequately dosed substitution therapy, such as methadone or a long-acting form of methadone, L- $\alpha$-acetylmethadol (LAAM), can serve an HIV prevention function.

Harm reduction policies have received more support in the last decade due to the spread of AIDS among injection drug users. The primary purpose of harm reduction is to decrease the negative consequences of drug use (83). As opposed to a policy of abstinence, harm reduction approaches realistically assume that some individuals will continue to use drugs. In this framework, a hierarchy of goals is established, with more immediate or attainable ones achieved on the way to risk-free use or possible abstinence. Risk reduction strategies such as methadone maintenance treatment, needle education and bleach distribution, safer sex education, legal clean needle purchase, and needle exchange programs are all examples of harm reduction strategies (84).

While injection drug use has a direct role in transmission of HIV, noninjection drugs and alcohol can play a potent role as cofactors of transmission because of their effects on behavior 
and cognition. The treatment success of substance use disorders varies, and the situation is further hampered because access to substance abuse services is often limited or is unwanted; relapses are common. Nonetheless, keeping substance use disorder intervention high on the list of treatment priorities is recommended for persons at risk for HIV infection.

One component of a comprehensive approach to HIV prevention among injection drug users is access to sterile syringes. The U.S. Public Health Service recommends that injection drug users who continue to inject use sterile syringes to prepare and inject drugs and obtain those syringes from a reliable source (e.g., a pharmacy). At the same time, a wide variety of laws and regulations restrict the ability of injection drug users to purchase and possess sterile syringes (7). Numerous national organizations, including the American Psychiatric Association (85), the American Medical Association, and the American Pharmaceutical Association, have recommended in policy statements and guidelines the removal of government restrictions on the availability of sterile syringes and have supported government-sponsored needle exchange programs. Cleaning drug injection equipment with bleach is an alternative that requires a multistep cleaning process that is impractical for many injection drug users (86).

\section{b) Patients with severe mental illness}

Rates of HIV infection among psychiatric inpatients averaged $7.8 \%$ in seroprevalence studies conducted in East Coast cities (87). Men and women were equally affected, with the highest rates occurring among patients who were under 40 , were black or Latino, or used substances, especially injected drugs. Rates for other geographic areas in the United States were not available.

Despite not identifying themselves as gay, $10 \%$ of men with schizophrenia have reported same-sex sexual encounters (88). Public mental health systems should implement prevention policies and practices, educate both mental and medical health care clinicians about key treatment issues, and develop effective linkages between clinicians and systems of care $(89,90)$.

Severe mental illness may be associated with health risks due to poor access to health care or decreased capacity to care for oneself. Despite the challenges that chronically and severely mentally ill patients face, risk reduction programs tailored to their needs have been shown to reduce risk of HIV infection $(91,92)$.

\section{c) Victims of sexual abuse/crimes}

Psychiatrists frequently encounter child, adolescent, and adult psychiatric patients who have histories of being sexually abused, including when treating patients with posttraumatic stress disorder (PTSD), dissociative disorders, and borderline personality disorder. Victims of sexual crimes vary from those with long abuse histories to those with a single sexual assault. Sexual coercion often results in long-term emotional damage to those that have been assaulted and may be followed by PTSD or other psychiatric disorders. These emotional scars are associated with increased vulnerability to other HIV-risk situations.

A patient with a history of sexual abuse or trauma should be asked about specific behaviors that are associated with risk for HIV transmission. Patients with such a history may be reluctant to provide information initially. Psychiatrists should determine if a psychiatric disorder is present and whether treatment is indicated. In the case of sexual assault, psychiatrists should expediently gather enough information so that the decision about the appropriateness of postexposure prophylaxis can be made (93).

\section{B. HIV-INFECTED INDIVIDUALS}

\section{Psychiatric management}

The development of a psychiatric treatment plan for patients with HIV infection requires thoughtful and comprehensive consideration of the biopsychosocial context of the illness. 
Treatment decisions must balance standard recommendations for psychiatric conditions against the medical stage of HIV illness and up-to-date information about available medical interventions targeted at the underlying HIV infection. At the same time, psychiatrists should be aware that emotional reactions and conflicts can interfere with a patient's ability to follow medical recommendations and thus have a profound effect on physical status. Psychiatrists should carefully consider possible medical causes of psychiatric symptoms and whether a medical workup is indicated to rule out a potentially life-threatening illness due to HIV or HIV-related illness. Organ malfunction, synergism of side effects of drugs, and drug-drug interactions are important factors to address in the management of patients.

\section{a) Establish and maintain a therapeutic alliance}

Establishing an alliance involves, in part, recognition of a patient's understanding of his or her stage of illness and an evaluation of how he or she is coping with it. The exploration of cultural/ ethnic beliefs regarding psychiatric and HIV illnesses can also contribute to the formation of a solid alliance. Because of the potential for the patient to feel shame and stigma associated with HIV infection and the sensitive nature of discussing risk behavior, psychiatrists should be supportive and not judgmental to encourage trust.

Issues of confidentiality should be reviewed with the patient, and the patient should be asked to consider the psychiatrist's role in assisting with the process of disclosure of HIV status to appropriate persons (94). In establishing a therapeutic alliance, it is important to discuss with the patient whether he or she wants to extend the treatment relationship to include selected communication with the family or significant other(s). Lastly, given the importance of the therapeutic alliance and the emotional impact of issues related to HIV, the psychiatrist should be aware of transference and countertransference feelings as well as personal attitudes about HIV infection and how the patient acquired the virus.

\section{b) Collaborate and coordinate care with other mental health and medical providers}

Managing the health care needs of a patient with HIV infection can be challenging due to the complex nature of the illness. Psychiatrists must be aware that the illness changes over time and has many different clinical manifestations. In addition, because information about HIV-related treatment is constantly evolving, psychiatrists may feel that their fund of knowledge about the most current interventions, such as antiretroviral medications, is inadequate. To keep up to date and to provide good clinical care, it is essential to collaborate with other physicians in infectious disease, primary care, and other disciplines.

Discussions of drug-drug interactions and the close monitoring and workup of unexplained somatic or psychiatric symptoms are examples of how psychiatrists and primary care physicians can assist each other in providing high-quality comprehensive care. Patients should specify their agreement for the exchange of specific information between the psychiatrist and other clinicians in a written release of information. It is often appropriate to use a multidisciplinary team approach when managing a patient with HIV, especially as the disease becomes more advanced. Access to both general medical and specialty care may need to be addressed.

\section{c) Diagnose and treat all associated psychiatric disorders}

A number of surveys of persons with HIV infection have shown an elevated premorbid rate of psychiatric disorders when compared to rates in the general population. In addition, psychiatric disorders can develop during any stage of HIV illness.

Psychiatric treatment of patients with HIV infection should include active monitoring of substance abuse, since it is often associated with risk behaviors that can lead to further transmission of HIV. Clinicians must not assume that patients who have relatively good immune functioning have no risk for CNS HIV disorders. Thorough evaluation and accurate diagnosis are key to selecting the appropriate intervention, whether it is risk reduction counseling, neuropsychological testing, or the use of psychotropic and antiretroviral medications. 
There are no data to suggest that the psychotherapeutic management of patients with HIV infection should be different from that of other patients. Many clinicians use a variety of approaches (e.g., both time-limited and longer-term individual and group psychotherapy) and psychotherapeutic models (e.g., cognitive behavior, supportive, interpersonal, or psychodynamic/ psychoanalytic).

\section{d) Facilitate adherence to overall treatment plan}

Adherence to a treatment regimen is profoundly important for patients with HIV infection. Research has demonstrated that less than $95 \%$ adherence to antiretroviral medications results in the development of viral resistance (95). Translated into actual practice, if medication doses are taken twice a day, a patient cannot miss more than one dose every 10 days.

Because comorbid psychiatric disorders, such as substance abuse or depression, have been shown to adversely affect patients' compliance with a complicated treatment regimen, psychiatrists and patients should actively discuss adherence to both psychotropic and HIV medications (96). Psychoeducational approaches are especially useful, since they reinforce the importance of adherence, support appropriate help-seeking, and identify barriers to adherence. Some patients who are unable to modify their behavior after educational approaches may be helped by intensive psychodynamic psychotherapy. If indicated, outreach efforts with public health nurses and services can be used to provide adherence assistance.

\section{e) Provide education about psychological, psychiatric, and neuropsychiatric disorders}

Mental health problems can occur at any stage of HIV illness (e.g., around the time of serologic testing) (97), or they could be precipitated by the onset of somatic symptoms. Preexisting psychiatric disorders or personality traits may be exacerbated by the onset of HIV illness. Patients may seek mental health services on their own, but it is not uncommon for other clinicians to request psychiatric consultation for patients who are in crisis or who have psychiatric symptoms.

It is often the psychiatrist's role to educate other clinicians and patients about the neuropsychiatric complications of HIV infection and to initiate and encourage treatment of current or emergent psychiatric disorders. When seeing a patient in consultation, it is important to gather history about cognitive or motor symptoms and conduct a mental status screening examination to determine whether neurocognitive deficits are present (see Section III.B.2.a on screening exams).

\section{f) Provide risk reduction strategies to further minimize the spread of HIV}

Psychiatrists are obligated to assess the risk for HIV transmission from their HIV-infected patients to others and to provide risk reduction counseling. This task should be a long-term treatment priority, since many HIV-infected individuals continue risk behaviors. Risk assessment should be repeated when there are changes in the patient's clinical status or social situation, such as the onset of binge drug or alcohol use or new sexual relationships. Psychotherapy may help some individuals who are unaware of motivations that promote ongoing risk behavior. When a psychiatrist cannot provide the specific risk reduction intervention that is indicated for a patient, he or she should refer patients to resources such as HIV/AIDS service organizations and support the intervention when initiated.

\section{g) Maximize psychological and social/adaptive functioning}

Biomedical interventions have stemmed the progression of HIV illness so that its course has increasingly resembled that of other chronic medical illnesses. Therefore, maximizing psychosocial functioning is relevant to the long-term social and economic impact of HIV infection. Psychiatrists can enhance a patient's functioning by helping him or her cope with the illness. Psychiatrists should ask about and be aware of a patient's use of alternative or complementary treatments, including herbal remedies. 
Assessment of social supports, utilization of appropriate community-based services, and resolution of financial and occupational concerns are all potential fruitful domains of inquiry. Many patients find support groups for persons with HIV infection helpful in coping with their illness, whereas others may prefer individual, couples, or family therapy.

\section{h) Role of religion/spirituality}

Inquiry about the spiritual beliefs and religious faith of a person with HIV infection should be a regular part of a psychiatric assessment and treatment planning, since they can be an important source of support for many with HIV/AIDS. Facing a serious illness often serves as a catalyst for a search for meaning and a renewal of spiritual beliefs and practices. A person's religious history includes not only current beliefs and practices but also religious traditions of one's family of origin and ethnic culture.

Religious congregations have had different, sometimes negative, responses to the spiritual needs of members with HIV/AIDS that often correspond to the core beliefs of the faith communities (98). Many ethnic minorities find the religious community or body of the church to be the network that most effectively addresses their need for support in crises.

The concept of spirituality goes beyond religious considerations to encompass multidimensional and existential perspectives that are important in maintaining well-being for many persons with HIV infection. It is not uncommon for persons with HIV/AIDS to seek alternative modes of spiritual expression, such as meditation. An assessment of the spiritual needs of a patient involves questions around the person's concept of God, sources of strength and hope, significance of practices and rituals, and perceived relationship between spiritual beliefs and health status (99). Information gathered may help caregivers assist patients to better cope with their illness at all stages but particularly as a patient nears death.

\section{i) Prepare for issues of disability, death, and dying}

As HIV illness advances, a psychiatrist may be asked to help evaluate the need for reasonable work or school accommodations or the ability to return to work in line with the Americans With Disabilities Act. Patients with minor children may need assistance with disclosure of illness and the establishment of a custody plan in the event of parental death from AIDS. Psychotherapy may be very helpful in reducing the emotional distress and turmoil activated by approaching death.

The fundamental right of a patient with advancing HIV illness to make treatment decisions can be supported when issues relating to disability, death, and dying are discussed by the appropriate parties in a timely and ongoing fashion. Discussions about preferences for care should be initiated by physicians, since it has been reported that only $36 \%$ of patients with AIDS had spoken with their physician about their preferred treatment (100). It is recommended that patients with HIV infection draw up a living will to guide end-of-life decisions in addition to a durable power of attorney. Copies of these documents should be placed in a patient's medical charts and in the files of their primary and specialty physicians.

\section{i) Advice to significant others/family regarding sources of care and support}

The HIV patient's significant others — partner, family, and friends—are often collaborative partners in care and support who often shoulder a significant share of the clinical day-to-day care of an acutely or terminally ill patient with AIDS. They also are a rich source of collateral information about the clinical status of the patient. The psychiatrist needs to take care that the patient has given consent before speaking directly with family and significant others.

Intimate involvement with the patient and his or her illness can lead to mental health difficulties for the significant others as well. Referral to support groups for significant others affected by HIV may be helpful, as can encouragement to participate in HIV/AIDS advocacy organizations. Both can provide emotional validation and a degree of respite for a significant 
TABLE 14. Screening Examinations for HIV-Associated Cognitive Motor Dysfunction

\begin{tabular}{|c|c|c|}
\hline Instrument & Administration & Comments \\
\hline $\begin{array}{l}\text { Mental Alteration } \\
\text { Test }(102)\end{array}$ & $\begin{array}{l}\text { Clinician } \\
\text { administered }\end{array}$ & $\begin{array}{l}\text { Timed test; verbal version of the Trail Making Test; } \\
\text { alternates between numbers and letters }\end{array}$ \\
\hline $\begin{array}{l}\text { HIV Dementia Scale } \\
(103)\end{array}$ & $\begin{array}{l}\text { Clinician } \\
\text { administered }\end{array}$ & $\begin{array}{l}\text { Five sections cover memory registration, attention, } \\
\text { psychomotor speed, memory recall, and } \\
\text { construction }\end{array}$ \\
\hline $\begin{array}{l}\text { Executive Interview } \\
\text { Test (104) }\end{array}$ & $\begin{array}{l}\text { Clinician } \\
\text { administered }\end{array}$ & $\begin{array}{l}\text { A 10-minute, } 25 \text {-item bedside test of executive } \\
\text { function, including snout reflex, word fluency, } \\
\text { echopraxia, go/no-go task }\end{array}$ \\
\hline
\end{tabular}

other. Some significant others and family members may be best served by referral for psychiatric evaluation and treatment, including individual or family therapy.

\section{Diagnosis and treatment of disorders requiring specific psychiatric intervention}

\section{a) Dementia and the spectrum of cognitive disorders}

Cognitive complaints are not uncommon among psychiatric patients in general, but the evaluation of such complaints in a patient with HIV infection requires a comprehensive psychiatric assessment, formulation of a differential diagnosis, and possible medical workup. Symptoms of early cognitive changes due to HIV can be subtle and can differ from symptoms associated with cortical dementia such as Alzheimer's. For example, HIV-associated dementia, due to its subcortical localization, more commonly presents with psychomotor slowing rather than deficits in language or visual recognition. Psychiatrists need to be aware of these differences in clinical phenomenology in order to identify HIV-associated dementia at early stages.

The widely used Mini-Mental State (101) is not sensitive in picking up early HIV-associated cognitive motor symptoms. Alternative screening examinations have been proposed that identify symptoms more likely to be present with subcortical dementia (102-105). Psychiatrists should become familiar with the available screening examinations for cognitive motor impairment that are more specific for subcortical symptoms (Table 14). It has been found that patient self-assessment of cognitive status is not reliable (106). Therefore, psychiatrists should administer a baseline screening examination on every patient with HIV infection and plan to readminister the test on a regular basis as part of the treatment plan. If there is evidence of early cognitive impairment, formal neuropsychological testing is useful to more comprehensively document cognitive dysfunction as well as areas of relative cognitive strength.

Once cognitive deficits are identified, the psychiatrist should work in collaboration with infectious disease specialists, neurologists, or primary care clinicians to develop a plan for further workup. A magnetic resonance imaging scan often reveals no abnormality in patients with early dementia, so this technique is not useful in providing specific confirmation of HIV-associated dementia. The overall immunological status of the patient should be assessed if not already known.

Pharmacologic treatment of HIV-associated dementia consists of intervening with potent antiretroviral therapy that targets the underlying HIV infection with consideration of whether the agents adequately penetrate the CNS. For comorbid conditions such as depression, psychiatrists should consider prescribing antidepressant medications as they would for other medically ill patients. Last, for management of symptoms associated with HIV-associated dementia (e.g., agitation or fatigue), medications such as antipsychotic or stimulant agents, respectively, should be considered. 
Psychotherapy may be helpful for patients with mild to moderate dementia in order to help them understand, mourn, and adapt to this new impairment of functioning. Both medications and psychotherapy can thus improve the quality of life both for persons with HIV-related cognitive disorders and their significant others while also improving overall clinical outcomes.

\section{b) Delirium}

The evaluation of the cause of delirium in an HIV-infected patient requires the psychiatrist to be alert to multiple possible etiologic factors and be knowledgeable about specific diseases that are associated with HIV infection. Examples are hypoxemia due to Pneumocystis carinii pneumonia, uremia due to HIV nephropathy, or elevated ammonia levels due to cirrhosis. One of the most important factors is the multiple medications that HIV patients typically take, which often cause delirium or contribute to delirious states because of drug-drug interactions. Problems arising from such toxicity are often reversible. Patients with AIDS who reside in either nursing homes or assisted living facilities or who have been hospitalized and who develop delirium have been shown to have significantly shorter survival than AIDS patients without delirium $(107,108)$. HIV-associated delirium may present with symptoms that resemble classic mania or drug intoxication, thereby bringing patients to the attention of a psychiatrist for evaluation. Delirium in the context of HIV infection should be evaluated like delirium with other medical conditions (47). A psychiatrist should advocate for a complete medical/neurological evaluation for patients with HIV infection who present with an acute onset of psychiatric symptoms with no previous psychiatric history. A complete workup should include a toxicology screen, thorough neurological examination, laboratory evaluation, and brain imaging studies. A comprehensive assessment for infectious processes should be conducted and may entail lumbar puncture.

Management of delirium in the context of HIV infection includes judicious use of antipsychotic medications for symptoms of agitation or perceptual abnormalities such as hallucinations. Many clinicians use the newer, atypical antipsychotic agents due to their lower side effect profile.

\section{c) Mood disorders}

The management of disturbances in mood such as depression or mania for patients with HIV infection is similar to that for other patients with medical comorbidity. Fatigue and insomnia, frequent complaints in otherwise asymptomatic patients, are likely related to psychological disturbances such as major depressive disorder (109). In addition, the overall medical status of the patient should be assessed to take into account possible effects of concurrent illness or side effects of medications such as efavirenz. Psychiatrists should know all medications that a patient is taking. Choice of an antidepressant or mood-stabilizing agent may be influenced by the antiretroviral regimen in place, and doses may need to be adjusted if drug-drug interactions are likely. Psychotherapy should be recommended when indicated.

Manic syndromes are difficult to treat in HIV-infected patients for several reasons. First, mania may result from HIV infection (secondary mania) (33), AIDS-associated brain infections, neoplasms, or treatment with medications like steroids. In addition, manic syndromes can be related to comorbid substance use disorders, and case reports have documented manic symptoms induced by the antiretroviral agents didanosine and zidovudine $(110,111)$. Patients who experience their first manic episode later in the course of their HIV disease are less likely to have personal or family histories of mood disorders and are more likely to have dementia or neurocognitive slowing (34). Although the prevalence and incidence of mania associated with HIV are not well described, treatment studies suggest that traditional antimanic agents are effective and tolerated.

\section{d) Substance use disorders}

In the United States, substance use disorders are prevalent in the population of persons with or at risk for HIV infection, and treatment is a high priority. Because drug- and alcohol-dependent 
HIV-infected patients form a large reservoir for HIV in the United States, and because behavior that risks transmission of HIV is often associated with concomitant substance use, psychiatrists should be aware that by treating substance abuse, they may well be preventing HIV infection (112). Unfortunately, the number of injection drug users in the United States outnumbers the available treatment slots.

Treatment with methadone or LAAM (113) can be an important treatment component for persons with opiate dependence. Since the quality of such programs varies, psychiatrists should help identify the best program for their patients. Factors that often indicate higher quality include the use of higher doses of methadone or LAAM and a close working relationship with primary medical clinicians and associated psychosocial services. It should be noted that doses of methadone may need to be increased or decreased in accordance with the use of specific antiretroviral agents that can have an impact on the metabolism of methadone.

Psychiatrists may be primary providers of care in a variety of clinical settings for HIV-infected patients who also have a substance use disorder. Psychiatrists should either provide treatment for their patients with comorbid substance abuse or collaborate with high-quality substance abuse programs. Substance use disorders themselves are often associated with comorbid psychiatric disorders such as anxiety, depression, and psychotic symptoms. Treatment of these comorbid conditions can help stabilize patients who are attempting to achieve sobriety or abstinence.

\section{e) Anxiety disorders}

Many problems involving anxiety symptoms can arise in relationship to HIV illness. An example of a clinical anxiety problem without HIV infection is AIDS phobia. For persons infected with HIV, there are numerous points at various stages of the illness when anxiety about the future, physical symptoms, or clinical decisions can become overwhelming. Psychotherapeutic approaches to situational anxiety can help patients work through intense affects and provide a structure within which sound decisions can be made.

Anxiety disorders can precede HIV infection or arise as its consequence. Treatment of anxiety disorders among HIV-infected patients has not been well studied; thus, psychiatrists should apply standard pharmacologic treatments for anxiety disorders with caution. For instance, many benzodiazepines are contraindicated when patients are taking protease inhibitors, particularly ritonavir, since predicted pharmacokinetics suggest blood levels of these psychotropic agents will be greatly elevated. Thus, benzodiazepines should be given as a short-term intervention in most instances. Psychiatrists may need to adjust medication doses and consider medical setbacks when treating patients with prominent anxiety symptoms. Psychotherapy may be effective in managing anxiety while reducing the need for medications. PTSD is a possible outcome of sexual assault or abuse and may be a focus of clinical treatment for some patients with HIV infection.

\section{f) Psychotic disorders}

Psychotic symptoms in the context of HIV infection, particularly at advanced stages of illness, do not necessarily indicate a primary psychotic disorder, such as schizophrenia, but may arise from causes ranging from opportunistic infections, mania, HIV-associated dementia, or delirium. Evaluation of new-onset psychosis requires a careful medical/neurological workup.

There is no literature to suggest that the use of antipsychotic medication needs to be modified for HIV-infected patients who have good immune functioning and are not taking antiretroviral medication. For patients taking antiretroviral medications, it is important to be aware of drug-drug interactions and overlapping toxicities. In particular, the use of clozapine is problematic with both ritonavir and zidovudine, the former because ritonavir may elevate blood levels of clozapine, the latter because clozapine and zidovudine can each cause significant bone marrow suppression. 
In late-stage HIV infection, atypical antipsychotic medications are the first-line treatment because standard neuroleptic medications have been associated with very severe and difficult-totreat extrapyramidal side effects. Also, in late-stage HIV illness, the lowest effective dose of any atypical antipsychotic medication should be given, since lower doses are sufficient to achieve efficacy and necessary to help prevent side effects.

\section{g) Adjustment disorders}

These disorders are interspersed among other diagnostic categories and are differentiated on the basis of onset after an identifiable stressor. Adjustment disorders are associated with significant emotional or behavioral symptoms. Although they may arise from stressful life events such as testing HIV-antibody positive, they may indicate a subsyndromal state that will evolve into a severe psychiatric disorder if left untreated. Various forms of psychotherapy may be indicated to prevent progression to a more severe psychiatric disturbance.

\section{h) Sleep disorders}

Sleep complaints are common in HIV patients in psychiatric treatment. Sleep disturbances may arise from a psychiatric disorder such as depression or stem from complications of HIV infection. For instance, because pain is a frequent accompaniment of HIV-related illness and is often treatable, clinicians should intervene to alleviate pain that causes sleep disturbance. The antiretroviral medication efavirenz is associated with a high incidence of vivid dreams and nightmares.

\section{i) Disorders of infancy, childhood, and adolescence}

The presenting psychiatric problems of HIV illness in children depend upon factors such as the age and developmental stage of the child, HIV clinical stage, psychosocial situation, and individual vulnerabilities for psychiatric disorders. Although there are few studies in this area, psychiatric disorders are common among infected youth, with rates of about $30 \%$ for mood disorders and $25 \%$ for attention deficit hyperactivity disorder $(114,115)$. As mood and anxiety disorders are more likely to be overlooked by caregivers than disorders with prominent behavioral manifestations (externalizing disorders), extra vigilance is required by the psychiatrist.

Just as the standard of care for HIV intervention has changed for adults, so have the treatments for children infected with HIV. Children have increased survival rates and slower progression to AIDS with the use of antiretroviral medications.

Psychiatrists, especially child and adolescent psychiatrists, need to help support children who survive to adolescence to negotiate this complex developmental stage. Psychotherapy may be of particular help for adolescents who are dealing with issues of developing sexuality. For some adolescents, sexuality may be a reminder of their infection. For others, sexual risk behavior may be a reenactment of parental behaviors, a method of mastering their trauma, or a response to their anger concerning their ill health. Substance abuse is frequent $(33 \%$ in one study) and is likely to involve multiple drug use (116). The issues of risk behavior and autonomy have implications for HIV prevention, adherence to treatment, and effective coping with chronic illness. The family's understanding and capacity to respond in a supportive manner are essential — these adaptations will not only help the child or adolescent cope with the attendant biopsychosocial adversity, they may also influence morbidity and mortality. The importance of family dynamics for children and adolescents with chronic illness and handicapping conditions has long been recognized $(117,118)$.

\section{i) HIV-associated syndromes with psychiatric implications}

In the case of somatic syndromes that exist at the interface of medical and psychiatric disorders, psychiatrists can serve to integrate treatment approaches and promote interdisciplinary and interspecialty dialogue. Symptoms such as fatigue, weight loss, pain, and sexual dysfunction can 
be associated with HIV illness as well as psychiatric disorders. It is useful to avoid all-or-nothing, mind or body, approaches when evaluating such nonspecific symptoms. Good communication between psychiatrists and other physicians leads to better treatment decisions. Principles of palliative care apply when a patient is terminally ill and desires treatment that focuses on comfort and symptom relief.

Wasting syndrome generally occurs in patients with more advanced HIV illness and can be related to a number of physiologic disturbances, such as progressive HIV disease, hypogonadism, and gastrointestinal malabsorption. Loss of lean body mass is a strong predictor of increased mortality due to AIDS (119). Wasting is defined as loss of $>10 \%$ of ideal body weight.

Fatigue is a common, often chronic symptom in HIV disease, frequently associated with depressed mood and physical disability, particularly among patients with more advanced HIV infection or AIDS (120-122).

Patients report pain at all stages of HIV illness, but complaints tend to be more frequent and more intense at advanced stages of systemic illness $(123,124)$. Common painful symptoms stem from headaches, herpetic lesions, peripheral neuropathy, back pain, throat pain, arthralgias, and muscle and abdominal pain (125).

Sexual dysfunction has been reported to occur in both men and women with HIV infection. In men, hypogonadism can be treated with testosterone replacement (126). As a rule, testosterone more effectively treats diminished libido than erectile dysfunction.

\section{CLINICAL AND ENVIRONMENTAL FEATURES INFLUENCING TREATMENT}

\section{A. SOCIODEMOGRAPHIC VARIABLES}

\section{Race/ethnicity}

AIDS and HIV infection disproportionately affect both the African American and Hispanic populations in the United States. In 1998, African Americans accounted for $45 \%$ of AIDS cases reported that year while constituting only $12 \%$ of the total U.S. population. The same year, the rate of reported AIDS cases for Hispanics in the United States was 20\%, although Hispanics constituted only 13\% of the general population (Figure 5). In 1998, African Americans overtook whites as the group with the largest number of persons living with AIDS. The rates have been relatively constant in the Asian/Pacific Islander and American Indian/Alaska Native groups: about $1 \%$ of cases (Figure 6).

The statistics are even more skewed when classified according to both race and gender. For those cases of AIDS reported through 1998, 77\% of all women with AIDS were black or Hispanic; $81 \%$ of all children with AIDS were black or Hispanic (127).

Three interrelated issues seem to account for the high rates of HIV/AIDS in minority communities: 1) inequities in the general health status in economically disadvantaged minorities, 2) problems controlling substance abuse in minority communities, and 3) the role of substance abuse in the spread of HIV sexually and perinatally.

HIV prevention efforts must be tailored for specific ethnic/cultural groups so that their effectiveness can be enhanced. This point is highlighted by the high levels of stigma associated with gay or bisexual activity in the African American and Hispanic communities. The impact of religious beliefs (Pentecostal, Catholic) in these communities may also affect HIV prevention efforts due to negative views of homosexuality. 


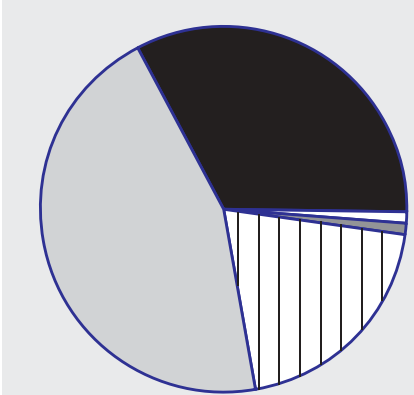

AIDS Cases

$(\mathbf{N}=\mathbf{4 8 , 2 6 9 a )}$

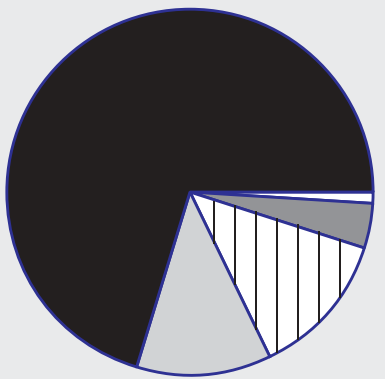

Population

( $N=274,766,000)$
$33 \%$
White, not Hispanic $71 \%$
$45 \%$
Black, not Hispanic 12\%
$20 \%$
Hispanic
$13 \%$
$1 \%$
Asian/Pacific Islander 4\%
$1 \%$
American Indian/ $\quad 1 \%$
Alaska Native

FIGURE 5. U.S. AIDS Cases Reported in 1998 and Estimated 1998 U.S. Population, by Race/Ethnicity (3).

${ }^{a}$ Includes 242 persons with unknown race/ethnicity.

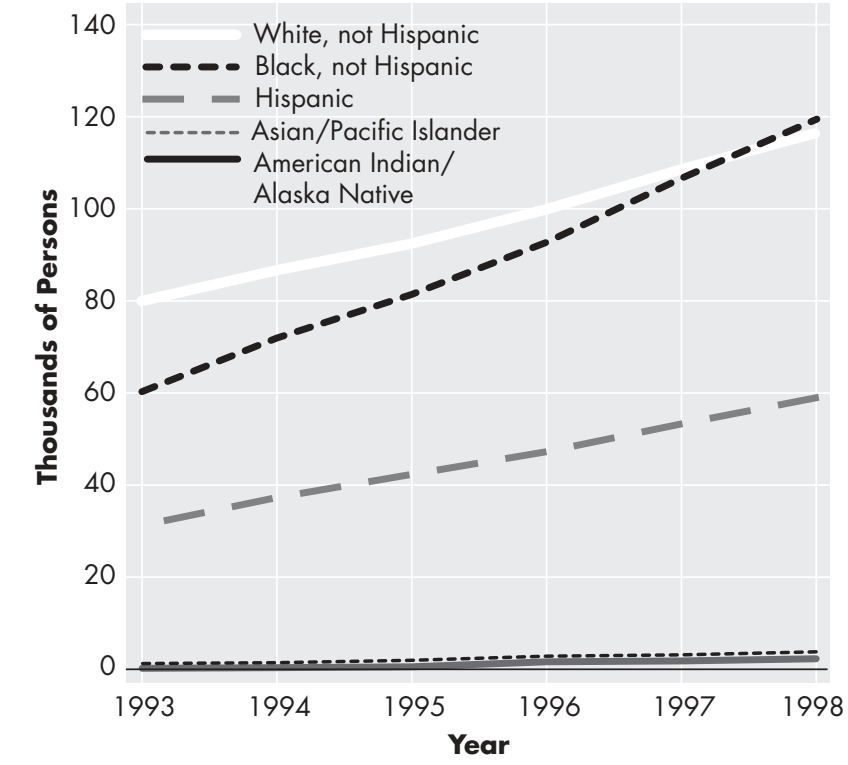

FIGURE 6. Estimated Number of Persons Living With AIDS in the United States, 1993-1998, by Race/Ethnicity (3). 
Hispanics in the United States constitute a diverse group of cultures and countries of origin. Surveys have found that exposure to HIV varies in different Hispanic subgroups. For example, injection drug use is more prevalent among Hispanics from Puerto Rico, whereas the more common risk behavior for Hispanics who originate from Mexico, Cuba, and South and Central America is unsafe sex among men who have sex with men (128).

\section{Sexual orientation}

Throughout most of the earlier years of the HIV/AIDS epidemic, men who had sex with men were disproportionately affected. Although this group still constitutes the population with the highest AIDS incidence (37\% of new AIDS cases in 1998), through massive prevention interventions, rates for men who have sex with men have declined, whereas other exposure categories are increasing annually. For example, in 1998, 12\% of new AIDS cases were linked to heterosexual transmission (127).

Evidence from HIV prevalence studies and risk behavior surveys supports the need to continue HIV prevention efforts among each generation of gay and bisexual men. There are cohort differences in attitudes, behavior, and perceived risk in younger and older gay and bisexual men. In one study of six urban counties, 5\%-8\% of adolescents and young men between the ages of 15 and 22 who were having sex with men were already infected with HIV (3).

Bisexual women have been reported to have significantly more sexually transmitted diseases and HIV risk than heterosexual women (129), and women who have sex with women constitute a group with a combination of risks for HIV infection. In general, female-to-female transmission is thought to be uncommon, although theoretically possible. The CDC writes that "of the 347 (out of 2,220) women who were reported to have had sex only with women, $98 \%$ also had another risk-injection drug use in most cases" (130). Women who have sex with women do need to know that exposure to mucous membranes, such as the vagina or mouth, or to vaginal secretions or menstrual blood could transmit HIV, especially if the concentration of the virus is high (early or late-stage HIV infection).

\section{Gender}

The percentage of women with both HIV infection and AIDS has been steadily rising over the past decade. AIDS cases among women and adolescent girls rose 364\% between 1991 and 1997. Trends in HIV diagnoses showed a 3\% decrease in men but a 3\% increase in women between 1995 and 1996 . Heterosexual contact is the leading risk category for women (38\%), with 29\% of cases in women resulting from personal injection drug use (127).

The exposure category "other/not identified" represents $32 \%$ of AIDS cases in women (127). It is typical for more recently reported cases to be classified as "other/not identified," but after medical record review and investigation, most cases are eventually attributed to one of the defined exposure categories.

African American and Hispanic women are disproportionately affected, with AIDS rates 17 and 6 times higher, respectively, than for Caucasian women. Despite the difficult socioeconomic conditions many minority women live in, a recent survey of health concerns among lowincome women found that AIDS was ranked as the most important concern (131).

There are issues that are unique to women and HIV/AIDS. Male-to-female transmission is estimated to be eight times more likely than female-to-male transmission (132). Sex differences in HIV measurements such as viral load, side effects of antiretroviral medications, and access to quality health care have all been documented (133). These biological differences make recommendations for antiretroviral therapies more uncertain for women. In addition, most clinical trials of interventions to treat HIV infection have enrolled male subjects. Women, particularly HIV-positive women, have psychological, psychosocial, reproductive, and gynecological concerns that differ from men $(134,135)$. 


\section{Age}

AIDS has always had the greatest impact on Americans ages 25-44. For example, in 1996, almost $75 \%$ of persons with AIDS were between the ages of 25 and $44 ; 21 \%$ were over age 44, and less than $4 \%$ were between the ages of 13 and 24 . More recent data have shown that one in four new infections in the United States occurs in people younger than 22 years of age (127).

Because of the impact associated with new medical therapies, many people's lives have significantly lengthened, which has led to older generations of persons living with HIV/AIDS. For instance, currently one out of 10 AIDS patients in the United States is over 50 years old; in Florida, one out of every six AIDS patients is older than 50 (136).

The age of diagnosis for $11 \%$ of AIDS cases is 50 years or older, and the age of diagnosis for $3 \%$ of AIDS cases is 60 years or older (127). However, there remains a common misperception that HIV is not a risk for older people. Many HIV-related symptoms mimic the effects of aging, so clinicians may be slow to diagnose HIV infection in older people and fail to question their older patients about risk behaviors (137). This is troublesome given research findings that document that adults over the age of 50 are up to one-sixth as likely to use condoms as people in their 20s (138). A particular diagnostic challenge for mental health clinicians treating HIVinfected older persons involves neurological symptoms, for which the differential diagnosis of cognitive impairment should be broadened to include dementias seen in older age.

\section{Economic factors}

According to data from the first national multisite HIV/AIDS Mental Health Services Demonstration Program, of nearly 2,000 persons with HIV/AIDS seeking mental health services, a majority were socially and economically disadvantaged (i.e., marginally employed, poor, relying on public assistance, or under- or uninsured) (139). Almost half of the 2,000 patients treated in this program lacked medical insurance, and for all patients median monthly income was only $\$ 575$. Other sources of economic information yield seroprevalence data that suggest higher rates of HIV infection among the poor. For example, among Job Corps participants (who are primarily economically disadvantaged youth), HIV seroprevalence rates are twice that of youth seen at adolescent clinics and eight times higher than rates for similar age youth who apply for military service (140).

\section{Urban versus rural}

Although HIV has historically disproportionately affected urban areas, rural populations are increasingly at risk. Although more drug-related risk behaviors are reported by residents of large metropolitan areas, high-risk sexual behaviors have not been found to differ significantly among urban and rural residents (141). However, one recent study found rural low-income women less likely to use condoms than their urban counterparts (142). When compared to urban dwellers living with HIV/AIDS, rural inhabitants report significantly lower life satisfaction, lower perceptions of social support from family and friends, reduced access to medical and mental health care, elevated levels of loneliness, more community stigma, heightened personal fear that their HIV status might be disclosed by others, and more maladaptive coping strategies (143).

\section{B. CLINICAL FEATURES}

\section{Suicidality}

Suicide, attempted suicide, and suicidal ideation are complex clinical issues that may arise during the course of psychiatric care for HIV patients (144). Studies in the 1980s, before antiretroviral treatments, reported rates of completed suicide in HIV-positive men as high as 66 times 
that of the general population (145-147). However, more recent data show suicide rates only modestly elevated and comparable to those in other medically ill populations (148), although perhaps most elevated among men who have sex with men (149). A study among U.S. military service applicants found suicide rates for both HIV-positive and HIV-negative individuals that were only marginally more elevated than those in the U.S. general population (150).

A number of risk factors for suicide in HIV patients have been described. They include psychiatric morbidity such as substance abuse $(151,152)$, antisocial personality disorder (153), family psychiatric history and family history of attempted suicide (154), and major depressive disorder. Numerous psychosocial factors have also been identified, such as a history of multiple HIV-related losses, lack of social support, loss of employment or insurance coverage, painful and disfiguring physical deterioration, exhaustion of financial resources, and reliance on public assistance $(155,156)$.

Recent evidence has shown that patients with greater levels of "fighting spirit," a specific pattern of adjustment to life-threatening illnesses, tend to have lower levels of suicidal ideation. Evaluation of suicide risk and other violent acts is an integral part of a comprehensive evaluation for HIV/AIDS patients. Psychiatric treatments that promote greater adaptation to living with HIV infection may lower the risk of suicide for many patients (154).

\section{Bereavement}

There are unique features to bereavement in the context of HIV/AIDS. Deaths from AIDS often disproportionately affect specific segments of the U.S. population. For example, it is not uncommon for a member of the gay community to have lost multiple persons to AIDS. At the same time, a bereaved individual may himself or herself be infected with HIV. Reports have suggested increases in high-risk sexual behavior and suicidal ideation in partners of individuals who have died of AIDS $(157,158)$. Bereavement is often compounded by the fact that many people die from AIDS at a young age, out of synchrony with the normal developmental sequence $(159,160)$.

Growing numbers of children will lose one or both parents to HIV. Psychiatrists need to explore the capacity of families to communicate about HIV and the children's understanding of their parent's illness. Disclosure is difficult for many parents who worry about revealing stigmatized behaviors or overwhelming a child with information (161). Permanency planning is an essential step in communication and collaboration with social agencies. For many children, bereavement is complicated by the psychosocial problems that existed before HIV that are then compounded by disintegration of the immediate family. Problem behaviors in adolescents who anticipate losing one or both parents to AIDS have been described (162). Family-based, multidisciplinary team approaches may be effective in providing access to care and needed followup services.

Researchers have studied the relationship of AIDS bereavement and psychological or medical variables but have not necessarily found that greater numbers of AIDS losses are associated with a higher rate of depressive symptoms or depressive disorders (163) or that distress increases in a dose-response relationship to the number of losses (164). One investigation reported changes in measures of immune function relevant to HIV disease in bereaved HIV-positive men that suggested patterns consistent with disease progression (165). Bereavement has been associated with decreased lymphocyte proliferation $(164,166)$ and decreased natural killer cell function $(167,168)$.

Group therapy is often recommended for AIDS-related bereavement (169). Group participants include partners, friends, family, and caregivers of persons who have died of AIDS. A randomized, controlled trial of support group interventions for bereaved HIV-positive and HIVnegative men demonstrated significant improvement in grief symptoms for the treatment group compared to control subjects (170). 


\section{Axis II disorders}

Several studies have reported that patients with HIV infection have a prevalence of personality disorders in the range of $19 \%-37 \%$, which is higher than rates found in comparable patients who are not infected (171). Personality disorders categorized (per DSM) within cluster B, such as borderline and histrionic personality disorder, are the most common (172). These data are supported by clinical reports from settings that provide care for large numbers of HIV-infected patients (173). The findings are not surprising given the association between personality disorders - especially those disorders in which "impulsivity" is prominent—and risk factors for HIV infection such as high-risk drug use and sexual behaviors. Personality disorders among those infected with HIV are associated with a higher rate of depression, maladaptive coping, and other psychiatric symptoms $(174,175)$ as well as with a higher rate of injection drug use (176). In fact, one study has documented that among their sample of HIV-positive and "at risk" HIV-negative gay men, persons with personality disorders were at significant risk of onset of future axis I disorders and serious functional impairment, regardless of a past history of axis I disorders (177).

No studies have assessed the efficacy of specific treatments for personality disorders among individuals infected with HIV. There is no evidence against the use of standard psychiatric treatments for personality disorders, tailored to the individual patient. Some clinicians use an approach that includes limit setting, especially limits on the inappropriate use of medical resources and medications. Other strategies for complex cases emphasize the development and maintenance of a doctor-patient relationship, treatment of comorbid conditions such as depression and substance abuse, and supportive or cognitive psychotherapy techniques (173).

\section{Psychoneuroimmunology}

The relationship of behavior and psychopathology to immune measures has been the focus of ongoing research over recent years. With regard to HIV infection, the findings are mixed. On the one hand, a number of studies have found that stressful life event burden is associated with decreased CD4 cell count (178-180), decreased cytotoxic T lymphocyte count (181), clinical HIV disease progression $(182,183)$, and physical symptom burden (168). In contrast, the relationship of variables such as stressful life events and depressed mood levels with markers of immunological progression has not been supported by other studies $(184,185)$.

There appears to be an increase in the frequency and severity of depression around the time of the onset of AIDS that persists throughout the time of severe immunodeficiency (66). Whether depressed mood levels in general are associated with decreases in immune function is not clear, since one study has shown such an association (186), whereas another has not (187). The disparity across studies attempting to elicit such natural history relationships may be due to methodological differences related to the extent to which other factors, including immunological measures and clinical disease progression, had been controlled (179).

Interventions such as cognitive behavior stress management, coping skills enhancement training, massage therapy, existential therapy, bereavement support groups, and antidepressant medication have all been evaluated with regard to their effect on immune measures (188-190). The findings are mixed, with some interventions showing modest improvements of specific immune measures, whereas others did not result in positive changes. Most interventions are safe, including those that involved the antidepressants fluoxetine and imipramine (190). At this time, these types of interventions should be regarded as investigational in terms of their immunological and health effects. However, psychotherapeutic and psychopharmacologic interventions are indicated for their alleviation of distress and enhancement of functional status.

A caveat regarding the use of such interventions is that many patients hold beliefs that may lead to inappropriate expectations of the potential for immunological and clinical health effects. Patients who do not observe these effects may come to blame themselves (and their be- 
havior) for the lack of such immunological and clinical effects and for disease progression. This could actually result in increased distress. Hence, patients must be carefully prepared for understanding the context in which behavioral interventions are being offered and which outcomes should be considered primary (psychological and functional) and which might be considered secondary and investigational (immunological and clinical health). On the other hand, clinicians should not discourage use of adjunctive techniques that can potentially add to the effect of current medical management by expanding the comprehensiveness of care.

\section{SPECIAL TREATMENT SITUATIONS}

\section{Alternative and complementary treatment}

Alternative and complementary medicine is an important part of the health care sought by many Americans. Surveys of the U.S. public in both 1990 and 1997 demonstrated that over one-third of Americans used at least one unconventional treatment in the past year, with rates rising from $34 \%$ to $42 \%$ between 1990 and 1997. It is estimated that the number of visits to nontraditional care clinicians exceeds total visits to all U.S. primary care physicians and represents $\$ 27.0$ billion in annual out-of-pocket expenditures $(191,192)$. More than $60 \%$ of patients who use complementary/alternative treatments do not disclose the treatments to their doctors. Depression is one of the most common conditions for which patients seek alternative/ complementary therapies.

Surveys specific to the HIV-infected population report similar findings, with between $30 \%$ and $68 \%$ of respondents reporting use of some form of complementary/alternative treatment (193-196). Patients with HIV/AIDS use complementary and alternative medicine to improve general health or quality of life, prevent progression of HIV disease, treat specific symptoms, or counter side effects that result from biomedical treatments $(197,198)$.

According to data from the first 1,016 participants of the Alternative Medical Care Outcomes in AIDS Study, the 10 most frequently used activities are aerobic exercise (64\%), prayer (56\%), massage (54\%), needle acupuncture (48\%), meditation (46\%), support groups (42\%), visualization and imagery (34\%), breathing exercises (33\%), spiritual activities $(33 \%)$, and other exercise (33\%) (199). Other nontraditional treatments include nutritional, vitamin, or herbal supplements and medicinal use of marijuana and anabolic steroids. Only one research study to date has examined complementary therapy in the context of HIV infection. A multisite project is currently being conducted in cooperation with the Office of Alternative Medicine, National Institutes of Health, to evaluate these treatments (200).

\section{Institutional settings}

Institutions that house, treat, or incarcerate have a responsibility to address issues of HIV education, prevention, and clinical services for persons served in these settings. Seroprevalence studies in institutional settings identify the scale of HIV infection and help inform policies for the institution. For example, concerns about HIV infection among institutionalized mentally retarded persons have been somewhat allayed by studies that found either no cases of HIV infection (201) or low rates $(0.16 \%)(202)$. These institutions nonetheless need to maintain universal blood and body fluid precautions as well as develop prevention programs tailored to their patient population.

Some of the highest rates of HIV infection are found among jails and prisons due to the high frequency of drug-related sentences and high-risk behaviors that occur within the institutions. Prevalence rates among male inmates have been recorded between $6 \%$ and $8.5 \%(203,204)$; one study of incarcerated women in New York found an 18.8\% rate of HIV infection (205). 
Despite the alarmingly high rates of HIV infection among prisoners in the United States, administrative and institutional barriers, such as not allowing the distribution of condoms, undermine prevention interventions. Collaboration between institutional staff and clinicians or researchers of HIV prevention efforts may lead to effective interventions for inmates and their partners (206). Psychiatrists working in correctional facilities may need to advocate for patients who may not receive antiretroviral medications in a timely manner due to lockdowns, solitary confinement, or other factors. Recommendations for handling HIV-related issues in psychiatric institutions can be found in policy and position statements issued by the APA Office on AIDS (www.psych.org/aids; click on "Policy Statements").

\section{Health care clinician issues}

HIV creates unique demands on clinicians. Stresses that are associated with the care of HIVinfected patients include fear of contagion, the emotional impact of dying patients, stigma associated with AIDS, anxiety concerning sexual and drug risk behavior, a constantly changing knowledge base, and often inadequate treatment resources (207). Health care workers have numerous reactions to these stresses. Clinicians have reported, for example, greater anxiety, more interference with nonwork activities, and symptoms similar to PTSD when treating AIDS patients as compared to non-AIDS patients $(208,209)$.

Despite the stresses and demands, most clinical staff members feel positively about their work, and only a small minority experience job burnout. The frequently cited rewards of working with HIV-infected patients include a sense of personal helpfulness, increased professional mastery, altruism, and admiration for patients' courage (210). In order to support employees, most multidisciplinary programs address issues that are likely to cause stress, such as death and dying, the management of sexuality, and fear of contagion. Interventions to address these issues range from weekly informal team meetings to leader-based staff support groups $(211,212)$.

Workshops that allow caregivers an opportunity to express grief, explore frustrations, and share resources may be helpful (213). Progress in HIV therapeutics requires frequent educational updates. Appropriate resolution of staff conflict and disagreement is useful in the HIV care setting, as it is in other settings that deal with chronic medical illness. One study, for example, found that HIV health care workers who perceived colleagues as significantly stressful were nearly $50 \%$ more likely to experience job burnout (214). Thus, a well-functioning HIV team may help buffer the stresses of providing care, and psychiatrists can be effective by assisting those teams.

\section{PART B:}

\section{REVIEW AND SYNTHESIS OF AVAILABLE EVIDENCE}

\section{DATA REGARDING PREVENTION FOR INDIVIDUALS AT HIGH RISK FOR HIV INFECTION

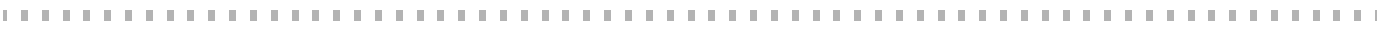

\section{A. HIV-RELATED RISK ASSESSMENT OF BEHAVIOR}

In a national survey of 417 practicing psychiatrists conducted in 1997 by APA, the physicians reported that over $9 \%$ of patients they treat are at risk for HIV infection (215); the rate was 
derived from questions about psychiatrists' knowledge of patient risk behavior. This estimate is probably conservative, since many psychiatrists do not conduct a detailed HIV risk assessment. Nonetheless, the survey data support other findings that psychiatric patients report higher rates of HIV risk behavior than the general population (141) and underscore the important role that psychiatrists play in preventing HIV infection.

Studies of primary care clinicians who conduct HIV risk assessments among patients who express concern about HIV risk found that in $73 \%$ of the encounters, the physicians did not elicit enough information to adequately characterize patients' HIV risk status (216).

\section{B. HIV ANTIBODY TESTING}

It is estimated that only two-thirds of all HIV-infected people in the United States know their serological status. Hence, many do not know until they are diagnosed with AIDS. The CDC recommends routine voluntary HIV antibody testing of patients who are admitted to hospitals that have HIV seroprevalence rates of $>1 \%$ among admitted patients.

A multistate evaluation found that anonymous HIV testing was associated with earlier HIV testing and HIV-related medical care when compared to those who were tested confidentially (217). Concerns about HIV test confidentiality led to a study of testing trends in six states that initiated name-based reporting of HIV test results. The researchers did not find significant declines in the total number of HIV tests performed at counseling and testing sites other than those expected from trends present before name-based reporting (218). Moreover, Osmond and colleagues (219) surveyed nearly 2,000 HIV-seropositive patients diagnosed during 19951996 in five states with name-based HIV surveillance (and three without) and reported that keeping records of people infected with HIV by name does not help or hinder treatment.

Home collection testing for HIV provides a method for testing among individuals who may not otherwise seek HIV testing through publicly funded sites or medical providers. A review of data collected by test manufacturers in the first year of availability (1996-1997) reported that of 174,316 tests performed, $0.9 \%$ were positive. Most users of home collection tests were white men aged 25-34 years; HIV prevalence was highest among nonwhites, men who had sex with men (particularly bisexual men), and injection drug users. Nearly $60 \%$ of those who submitted samples and $49 \%$ of those who tested positive had never had a previous HIV test (220).

\section{INTERVENTIONS TO REDUCE HIV RISK BEHAVIOR}

A wealth of literature addresses the issue of how to intervene to reduce HIV risk behavior at both the individual and community level. While this section will focus primarily on data concerning interventions at the individual level, macro-level changes in structure or policy can lead to powerful community-level support of specific behavior changes (221). The AIDS Community Demonstration Project is a good example of a community-level intervention. Community volunteers and "small" media were able to influence community norms about the use of condoms and disinfection of drug injection equipment by using bleach (222). Other examples of societal-level policies that influence the effectiveness of individual-level HIV risk behavior interventions are 1) laws that prohibit same-sex marriage, which hinder the establishment of long-term monogamous relationships for men who have sex with men (223), and 2) insufficient substance abuse treatment capacity, which decreases the opportunity for an injection drug user to receive treatment.

Sexual behavior is the target of most prevention efforts worldwide, but because of the diversity of sexual behavior and the influences of economics, culture, and environment, interventions to stem HIV necessarily vary as well (224). Because of the sheer size of the prevention literature and the many specific populations that have been targeted, these guidelines will not 
provide more detailed information about subgroups based on gender, ethnicity, or age. A publication from the CDC entitled "Compendium of HIV Prevention Interventions with Evidence of Effectiveness" provides a useful synopsis of the research in this area (225).

\section{Counseling strategies}

The HIV counseling and testing model has been widely used in studies that intend to promote behavior change in persons at risk for HIV infection. Yet, two large reviews of HIV counseling and testing studies $(226,227)$ found that HIV counseling and testing interventions do not consistently lead to risk reduction or promote help-seeking behavior in most populations studied. Both analyses found that serodiscordant heterosexual couples demonstrated the most change in risk behavior. A recent meta-analysis of the effect of HIV counseling and testing interventions found that they were more effective in secondary prevention for HIV-positive persons than as a primary prevention strategy (228).

The most widely used counseling models acknowledge that knowledge about HIV transmission and prevention is necessary but not sufficient for behavior change (229-231). More effective interventions rely on skills training and take into account individual psychosocial and cognitive strategies (232). Essential to effective counseling is helping patients perceive themselves as at risk for HIV infection, addressing motivation to reduce risk, and ensuring that patients have the skills and resources to implement risk reduction strategies. Changing peer norms with the help of peer leaders and matching the background characteristics (e.g., race, ethnicity, sexual orientation) of the leader to the targeted population often help increase intervention effectiveness.

The largest randomized, controlled HIV behavioral intervention study conducted in the United States found that even among persons served in public health settings, a small-group, seven-session risk reduction intervention cut reported high-risk sexual behaviors in half and more than doubled reported regular use of condoms (from $23 \%$ to at least 60\%) (233). HIV prevention interventions have proven useful for psychiatric populations, including injection drug users and people with severe mental illness, and have been shown to be effective even when conducted in busy public clinics $(234,235)$.

\section{Specific strategies for injection drug users}

The principles for preventing the spread of HIV among active drug injectors are similar to those used to modify unsafe sexual behavior. Ethnographic research has clarified how injected drugs are purchased, dissolved, filtered, transferred into syringes, and injected, and how sharing practices at each step in the process contribute to the potential spread of HIV (236). Successful interventions focus on knowledge, motivation, and behavioral skills that are tailored to realistic strategies for changing sharing practices. These programs have also engaged members of the injection drug use community as prevention leaders.

An international study found that injection drug users are capable of changing their risk behavior and providing accurate reports of their behavior. Further, these behavioral changes are associated with lower rates of HIV infection (237). A review of the research literature on HIV prevention outreach efforts concluded that most such programs have led to changes in drugrelated and sex-related risk behaviors in injection drug users (238). Specific reductions in drug injection, sharing of injection equipment, and crack use and increases in needle disinfection, condom use, and entry into drug treatment were seen with such programs.

The most controversial component of HIV transmission prevention in the injection drug use population has been the use of needle and syringe exchange programs that make sterile equipment available to drug injectors. These programs have met strong political opposition in many countries, including the United States, and assessment of their effectiveness has been fraught with methodological difficulties. There are unsubstantiated concerns that exchange programs may at- 
tract subjects who engage in the riskiest practices and that the number of needles or syringes in circulation may not be sufficient to ensure single-time use (239). Recent U.S. and international data make a strong argument for the benefits of needle exchange programs on reducing HIV transmission as well as other parenterally transmissible infections such as hepatitis B and C (240, 241). Nonetheless, since federal funds to date have been prohibited for use in needle exchange programs, they have had limited impact on lowering HIV transmission in the United States. Needle exchange programs that do exist are often small and cannot meet local demand.

\section{REDUCING MATERNAL-FETAL TRANSMISSION}

Before interventions with antiretroviral treatment were instituted, between 1,000 and 2,000 infants were born each year in the United States with HIV infection (242). Since recommendations were made for routine counseling and voluntary HIV testing of pregnant women, and for subsequent treatment with zidovudine for those who prove seropositive, the CDC has recorded dramatic decreases in perinatal transmission. From 1992 to 1997, cases of perinatal AIDS declined 67\%, including an 80\% decline in AIDS cases among infants and a $66 \%$ decline among children between the ages of 1 and 5 (243). It has been shown that elective cesarean delivery may further decrease the risk for fetal infection (244).

The protocol of administering zidovudine to a pregnant woman in the last two trimesters, followed by intravenous zidovudine during delivery, then 6 weeks of zidovudine treatment for newborns was found to reduce the incidence of HIV transmission from mother to child from $22.6 \%$ to $7.6 \%$ - a $66 \%$ reduction $(245,246)$. While the mechanism by which zidovudine reduces perinatal transmission is not known, research has shown that a risk factor for perinatal transmission is the amount of HIV in the pregnant woman's blood $(247,248)$. At the same time, the effect of zidovudine's lowering maternal HIV viral load does not fully explain the efficacy of zidovudine in reducing transmission. Another important component of protection may be preexposure prophylaxis of the fetus or infant to antiretroviral drugs. Because it has been shown to be metabolized into its active triphosphate form within the placenta, zidovudine may have distinct superiority in efficacy compared to other nucleoside analogues such as didanosine and zalcitabine (249).

There have been other studies of interventions to reduce perinatal transmission, such as shortcourse antenatal/intrapartum zidovudine in non-breast-feeding Thai women (250) or the combined agent lamivudine/zidovudine during gestation, intrapartum, and postpartum in African women (251). Both interventions were associated with reductions in perinatal transmission by approximately $50 \%$ (even though the group given lamivudine/zidovudine breast-fed). In the situation when the diagnosis of HIV infection is not made until near the end of gestation or during labor, treatment with the combined agent lamivudine/zidovudine or nevirapine in the intrapartum/postpartum period has been shown to be effective in reducing transmission $(251,252)$.

There are limited data on the effect of combination antiretroviral therapies on pregnancy outcome. A retrospective Swiss study reported that $80 \%$ of women experienced side effects to the drugs, which included reverse transcriptase inhibitors and protease inhibitors, and further, found a possible association with preterm birth (253).

Women who face decisions regarding the use of antiretroviral medications during pregnancy must consider the effects of treatment on their own health as well as on fetal or child health. These decisions are complex, since the standard of care has moved to multiple antiretroviral drugs, and yet the long-term consequences of exposure to such drugs by the infant are not known. In general, the three-stage zidovudine regimen (prenatal, intrapartum, and postpartum) alone or in combination with other antiretroviral agents should be discussed and offered to all HIV-infected pregnant women.

Antiretroviral protocols to prevent perinatal transmission are burdensome for patients and practitioners, and adherence is again a crucial factor in treatment effectiveness. A 1995 study 
found that only $15 \%$ of HIV-positive mothers and their newborns received the full zidovudine treatment regimen (252). This same study found that $26 \%$ of pregnant women with HIV infection did not receive any prenatal care. Coordination of care by providers of medical, mental health, drug abuse, and social services may be required for the successful implementation of antiretroviral treatment during pregnancy.

\section{E. POSTEXPOSURE PROPHYLAXIS}

While universal infection control precautions have become the standard of care in the U.S. health care system, as of June 1998, 54 health care workers had documented HIV infection through occupational exposure. Of the 54, 46 had percutaneous (puncture/cut injury) exposure, five had mucocutaneous (mucous membrane or skin) exposure, two had both percutaneous and mucocutaneous exposure, and one had an unknown exposure (82). The estimated risk of HIV infection in health care workers with percutaneous HIV exposure is approximately $0.3 \%$ (254).

Postexposure prophylaxis with antiretroviral agents has been shown to be safe and effective (82). The postexposure prophylaxis protocol outlines specific steps in assessing risk. First, the type of exposure-including type of material, contact surface type, volume, and severity of exposure-is determined. Next, the HIV status of the exposure source is assessed on the basis of factors such as probable viral titer (based on clinical HIV status of exposure source) and whether actual HIV status is known (it may be unknown). The exact postexposure prophylaxis recommendation - whether to intervene at all, use two agents (zidovudine and lamivudine), or use three (both of the above plus either indinavir or nelfinavir)—is based on these assessments. If done properly, postexposure prophylaxis can reduce the risk for occupational transmission of HIV infection in health care workers with known exposure, such as a needle stick injury involving blood or other body fluids, by $80 \%$ (255).

\section{F. SPECIAL PSYCHIATRIC POPULATIONS}

\section{Patients with substance use disorders}

$\mathrm{HIV}$ is spread both by sharing injection drug use equipment (most frequently practiced by opiate, cocaine, or methamphetamine users) and by engaging in unsafe sexual practices that are associated with substance use disorders. Documented sexual risk behaviors among those with substance use disorders include having multiple partners in the context of buying or selling sex for drugs or money to buy drugs; unprotected sex with injection drug users; and difficulty employing safer sex practices while intoxicated. These sexual encounters can also increase the risk of transmission of other sexually transmitted diseases that are cofactors for HIV transmission. Unfortunately, sexual risk behaviors are more resistant to change than drug-use risk behaviors in drug users (256).

Comorbidity of substance use disorders with other psychiatric disorders deserves mention. A study that used data from the 1992 National Survey of Veterans examined whether substance abuse or other mental disorders serve as risk factors for HIV infection. The investigators found that the combination of substance abuse and PTSD increased the rate of HIV infection by almost 12 times over that of those without either disorder (257).

Drug use disorders are associated with high rates of incarceration for drug-related crimes. Jails and prisons, in turn, bring people with elevated rates of HIV infection together in a confined space, increasing the chance that a sexual or injection drug use partner encountered in these settings will be infected with the virus $(258,259)$. Institutionalization in all-male settings also increases the likelihood of sex between men.

There are geographic differences in seroprevalence rates of HIV in injection drug use populations, with the highest rates in the United States occurring in the Northeast, on the East 
Coast, and in Puerto Rico (3). Holding the risk behavior constant, the likelihood of acquiring HIV increases with its penetration into a given geographic area by increasing the chance that sexual and drug injection partners will be infected. Because substance use disorders are associated with decreased occupational functioning or disability, many of these individuals become impoverished and often cluster in urban neighborhoods that are endemic for HIV.

\section{Patients with severe mental illness}

The severely and persistently mentally ill population is at risk for HIV infection due to factors analogous to those of patients with substance use disorders. There are high rates of substance use disorders among those with severe mental illness, and both populations share similar tendencies to reside in institutions or poor urban neighborhoods where cases of HIV infection are concentrated. Chronically mentally ill adults have been documented to have misunderstandings about AIDS transmission, to have histories of treatment for sexually transmitted diseases, and to report behaviors such as "survival sex" (in exchange for shelter, food, or money) (260). When compared to the general population, people with severe mental illness have higher rates of same-sex sexual activity and intimate contact with multiple partners (261). One study found an association of positive symptoms of psychosis and the diagnosis of schizophrenia with specific high-risk sexual behaviors (74).

Randomized, controlled trials of relatively brief AIDS prevention programs for the chronically mentally ill led to significant improvements in rates of unprotected intercourse and condom use (262) and knowledge about AIDS (263) in the intervention groups. It is not known whether such improvements persist beyond the short follow-up period.

\section{Victims of sexual abuse/crimes}

A history of sexual abuse is highly prevalent among both women and men living with or at risk for HIV infection. One report of 408 mostly economically disadvantaged women (at least 18 years old) found a lifetime prevalence of sexual abuse of $43 \%$; over one-half of the cases occurred during adulthood (264). A cohort of 327 homosexual or bisexual men had a $35.5 \%$ rate of childhood sexual abuse. Men with a history of childhood sexual abuse reported more lifetime male partners and a higher likelihood of unprotected receptive anal intercourse in the past 6 months (265). Women with a history of childhood sexual abuse have been found to have more sexual partners, heightened sexual activity, and more negative expectations of condom use (266). For example, a study of women in low-income housing developments found that those with a history of sexual coercion were likely to perceive that requesting a partner to use a condom would create a potentially violent situation (267).

In children, the occurrence of sexually transmitted diseases can be the first indication of sexual abuse. Although perinatal transmission of HIV accounts for the vast majority of HIV cases in children, HIV transmission has been reported to occur through childhood sexual abuse (268). Efforts to encourage active risk reduction in high-risk populations through AIDS education have found that adolescents who have been sexually abused are significantly less likely to use condoms and have much greater communication difficulties than their peers (269).

\section{DATA REGARDING PSYCHIATRIC TREATMENTS FOR INDIVIDUALS WITH HIV INFECTION}

Psychiatric treatments for individuals with HIV infection follow the same principles as for any patient with a psychiatric diagnosis, but there are special considerations. As with other patients 
with comorbid medical and psychiatric illnesses, it is essential to help patients cope with HIV illness and, when appropriate, with death and dying. There is also an ongoing need to determine whether new psychiatric symptoms have a medical basis that requires intervention. Examples of unique treatment issues with HIV patients include but are not limited to the following:

- working with the patient on disclosure of HIV status;

- assessing dangerousness from the perspective of HIV transmission and intervening to reduce that risk;

- helping families with dependent children arrange permanency planning;

- treating HIV-infected children who have developmental delays resulting from prenatal drug exposure or from HIV infection;

- negotiating disability status and, for some patients, a return to work;

- bereavement/loss; and

- treatment adherence.

\section{A. ACCESS TO SERVICES}

Most patients are asymptomatic for many years after becoming infected. But once symptomatic with more advanced HIV disease, they may become high users of medical care, including mental health services (270). They may need frequent assessment and treatment visits, undergo many medical tests, require hospitalization, and take multiple medications (often as many as 10-15 different medications at different times of day). Many patients receive medical care from an HIV primary care or infectious disease clinic or from an infectious disease specialist in the community. A recent study reported better medical outcomes for patients with HIV infection who receive care from physicians with more experience treating HIV diseases (271).

The Ryan White CARE (Comprehensive AIDS Resources Emergency) Act was enacted in 1990 to help states, communities, and families provide care for people with HIV/AIDS who lack adequate health insurance or other resources. Since its inception, CARE Act grants have totaled $\$ 6.4$ billion and have served approximately 500,000 individuals with HIV/AIDS annually. Administered by the Health Resources and Services Administration of the U.S. Department of Health and Human Services, CARE Act grants were reauthorized in 1996 and comprise several programs. Title I grants provide funds to cities for low-income or under- or uninsured persons to cover the cost of health care and medications, as well as support services like counseling and home and hospice care. Title II grants are for the 50 states, the District of Columbia, Puerto Rico, and U.S. territories to improve the quality, accessibility, and organization of both health care and support for patients with HIV/AIDS. Title III grants are given to public or nonprofit entities that provide comprehensive primary health care services for persons with AIDS or at-risk populations. Title IV grants fund efforts to coordinate HIV services and access to research for children, youth, women, and families. An additional component of the CARE program includes training for health care professionals, grants for innovative service delivery models for special populations, and dental services.

Despite CARE Act funding, lack of access to care remains a problem that affects morbidity and mortality for certain groups. One study documented that among urban patients who receive medical care, there are no differences in HIV disease progression or survival associated with sex, race, injection drug use, or socioeconomic status. This suggests access to medical care is a main determinant of how quickly a patient develops AIDS (272). More recently, investigators from the HIV Cost and Services Utilization Study, a national population-based sample, found that African American, Hispanic, female, uninsured, and Medicaid-insured individuals all had inferior patterns of medical care (e.g., later initiation of antiretroviral medication) than did Caucasian and privately insured individuals (273). 
Persons with HIV infection have higher rates of most psychiatric disorders than the general population. Psychiatric patients with common medical problems, especially those with severe mental illness, receive suboptimal medical care and suffer greater morbidity and mortality from medical illnesses than the general population. Studies are now under way to determine the access of patients with severe mental illness to HIV-related medical treatments; it can be assumed that these patients need help to access medical services.

Psychiatric disorders can be underdetected, misdiagnosed, undertreated, or treated improperly in primary care settings. For example, while a substantial majority of HIV-infected persons presenting for intake to an HIV primary care clinic suffered from depression, substance dependence, or another current psychiatric disorder, most cases were not detected by clinicians (66). Underscoring the importance of mental health care is the finding that ongoing high-risk sexual behavior is predicted by higher levels of depression and recreational drug use (274).

There are several patient barriers to meeting the mental health needs of HIV-infected persons. These barriers include adherence difficulties (e.g., missed appointments or inability to follow treatment recommendations); fear of additional stigmatization (the "double stigma" of HIV and a mental disorder); negative prior experiences with the mental health system; lack of funds for transportation, payment of care, or medications; unstable housing and frequent relocation; and the complex comorbid presentations of patients with HIV infection (e.g., depression complicated by concurrent substance dependence and a personality disorder). On the other hand, health care professionals should not assume that depression is "normal" in a person with HIV infection.

Some innovative HIV clinics integrate medical, mental health, and substance abuse treatment within their programs (275). These model programs increase the opportunities for prevention and treatment and may reach a more impaired population (276). One model uses an outpatient psychiatric consultation-liaison clinic that specializes in the treatment of HIVinfected patients. The clinic can be physically off-site from an HIV clinic but receives most of its referrals from that clinic. Other models include HIV programs within community mental health centers, programs located within residential substance abuse treatment facilities, freestanding community-based agencies, and referrals to private practitioners with expertise in treating HIV patients. All of these models appear to be effective (67).

\section{B. PHARMACOLOGIC TREATMENT}

In general, the use of psychotropic agents in HIV patients follows similar principles for using these medications in such populations as geriatric patients or those with comorbid medical illnesses. This is particularly true for patients with more advanced HIV disease and those on complex antiretroviral regimens who may be more sensitive to medication dosages and side effects and are at higher risk for drug-drug interactions (277). Because some standard medications used to treat HIV infection or HIV-related conditions can potently inhibit or induce the cytochrome P450 system, psychotropic medications that share metabolic pathways should be used judiciously; however, this concern should not preclude appropriate pharmacologic intervention.

General guidelines, particularly for patients with symptomatic HIV disease, include 1) using lower starting doses and slower titration, 2) providing the least complicated dosing schedules possible, 3) focusing on drug side effect profiles to avoid unnecessary adverse events (e.g., anticholinergic effects from tricyclic antidepressants, leukopenia from carbamazepine), and 4) maintaining awareness of drug metabolism/clearance pathways to minimize drug-drug interactions and possible end organ damage.

Published reports of adverse events and side effects can offer specific guidance for the use of some psychotropic agents in HIV-infected patients. However, clinicians must be cautioned that many studies that have examined psychotropic effectiveness in HIV patients were conducted on relatively asymptomatic patients, often did not include women or youth, and were conducted before the availability of combination antiretroviral therapy. Liver disease, in particular hepatitis $\mathrm{C}$, 
TABLE 15. Cytochrome P450 Inhibition Potency of Protease Inhibitors, by Isoenzyme System

\begin{tabular}{ll}
\hline Protease Inhibitor & Cytochrome P450 Isoenzyme Inhibited \\
\hline Ritonavir & $3 \mathrm{~A} 4,2 \mathrm{C} 9,2 \mathrm{D} 6$ \\
Indinavir & $3 \mathrm{~A} 4$ \\
Nelfinavir & $3 \mathrm{~A} 4,2 \mathrm{C} 19,2 \mathrm{D} 6$ \\
Saquinavir & $3 \mathrm{~A} 4,2 \mathrm{C} 9$ \\
\hline
\end{tabular}

is becoming increasingly important as a comorbid condition. Thus, routine liver function testing is necessary when prescribing psychotropic medications for patients with HIV infection.

Combination therapy with multiple medication classes constitutes the standard of care for successful treatment of HIV infection. Although limited clinical information is available concerning drug-drug interactions involving psychotropic and antiretroviral medications, effective psychotropic medication management in the setting of HIV requires attention to possible interactions.

Potential drug-drug interactions in the setting of antiretroviral therapy include the effects of antiretroviral agents on psychotropic medications and vice versa. Protease inhibitors and nonnucleoside reverse transcriptase inhibitors are metabolized by the cytochrome P450 system and may inhibit or induce multiple isoenzymes. For example, the protease inhibitor ritonavir inhibits the cytochrome P450 isoenzymes 3A, 2D6, and 2C9/19; thus, most psychotropic medications may be affected when concomitantly used with ritonavir. Other protease inhibitors tend to only inhibit the cytochrome $\mathrm{P} 450$ isoenzyme 3A, thus limiting possible drug-drug interactions to psychotropic medications metabolized via this pathway (e.g., some benzodiazepines, citalopram, and nefazodone).

The nonnucleoside reverse transcriptase inhibitors also require careful attention. For example, nevirapine and efavirenz are metabolized via the cytochrome $\mathrm{P} 450$ isoenzymes $3 \mathrm{~A}$ and $2 \mathrm{~B} 6$ and induce cytochrome $\mathrm{P} 450$ activity, which could result in decreased psychotropic concentrations at standard doses (278). Delavirdine, another nonnucleoside reverse transcriptase inhibitor, inhibits the cytochrome $\mathrm{P} 450$ isoenzyme $3 \mathrm{~A}$ and reduces hepatic clearance of psychotropic agents primarily metabolized by this pathway (279). The protease inhibitors and nonnucleoside reverse transcriptase inhibitors are among the most problematic of the antiretrovirals when coadministered with psychotropic medication. Table 15 indicates the relative rank order of inhibition potency of the currently available protease inhibitors.

For combination antiretroviral therapy that includes protease inhibitors and nonnucleoside reverse transcriptase inhibitors, an overarching concern in terms of coadministration with psychotropic agents is not only the possibility of plasma levels of psychotropic medications outside the targeted therapeutic concentration range but also the possible reduction of antiretroviral levels to the point of risking their effectiveness. While most interactions inbibit the cytochrome P450 system, some clinically important drug interactions also induce this system with medications used to treat co-occurring medical conditions in HIV disease (e.g., glucocorticoids, rifampin, phenytoin). Table 16 provides an overview of psychotropic agents and HIV therapies, routes of metabolic inhibition and induction, and potential clinical concerns.

Although the aforementioned risks for drug-drug interactions may seem substantial, to date, clinical data have not revealed severe side effects among most patients receiving antiretrovirals in conjunction with psychotropic medications (50). A few reports have documented the inhibitory effects of protease inhibitors. For example, when coadministered with ritonavir, desipramine levels have been shown to increase $145 \%$ in vitro (280) and to increase to some extent when coadministered with other protease inhibitors (281). In a clinical setting, coadministration of saquinavir and midazolam has caused prolonged sedation (282). These in vitro and in vivo data should lead to careful monitoring for potentially problematic or dangerous side effects in patients taking antiretrovirals. 
TABLE 16. HIV-Related Medications and Psychotropic Agents Involving the Cytochrome P450 System

\begin{tabular}{|c|c|c|c|c|c|}
\hline $\begin{array}{l}\text { Cytochrome } \\
\text { P450 Isoenzyme }\end{array}$ & $\begin{array}{l}\text { Common HIV-Related } \\
\text { Medications That } \\
\text { Inhibit Isoenzyme }\end{array}$ & $\begin{array}{l}\text { Psychotropic Medications } \\
\text { Primarily Metabolized by } \\
\text { Isoenzyme }\end{array}$ & $\begin{array}{l}\text { Possible Clinical Implications of } \\
\text { Isoenzyme Inhibition }\end{array}$ & $\begin{array}{l}\text { Common HIV-Related } \\
\text { Medications That } \\
\text { Induce Isoenzyme }\end{array}$ & $\begin{array}{l}\text { Possible Clinical Implications of } \\
\text { Isoenzyme Induction }\end{array}$ \\
\hline $3 \mathrm{~A} 4$ & $\begin{array}{l}\text { Protease inhibitors } \\
\text { (especially } \\
\text { ritonavir) } \\
\text { Delavirdine } \\
\text { Clarithromycin } \\
\text { Erythromycin } \\
\text { Itraconazole } \\
\text { Ketoconazole } \\
\text { Macrolide } \\
\text { antibiotics }\end{array}$ & $\begin{array}{l}\text { Benzodiazepines } \\
\text { Buspirone } \\
\text { Citalopram } \\
\text { Carbamazepine } \\
\text { Nefazodone } \\
\text { Trazodone }\end{array}$ & $\begin{array}{l}\text { Increased plasma levels and } \\
\text { increased side effects; } \\
\text { for benzodiazepines, } \\
\text { sedation and decreased } \\
\text { respiratory drive }\end{array}$ & $\begin{array}{l}\text { Nevirapine } \\
\text { Efavirenz } \\
\text { Glucocorticoids } \\
\text { Rifampin } \\
\text { Rifabutin }\end{array}$ & $\begin{array}{l}\text { Decreased plasma } \\
\text { levels of psychotropic } \\
\text { medications and } \\
\text { decreased effectiveness }\end{array}$ \\
\hline 2D6 & $\begin{array}{l}\text { Protease inhibitors } \\
\text { (especially } \\
\text { ritonavir and } \\
\text { nelfinavir) }\end{array}$ & $\begin{array}{l}\text { Mirtazapine } \\
\text { Fluoxetine } \\
\text { Paroxetine } \\
\text { Sertraline } \\
\text { Fluvoxamine } \\
\text { Tricyclic } \\
\text { antidepressants } \\
\text { Venlafaxine } \\
\text { Neuroleptics, } \\
\text { typical and } \\
\text { atypical }\end{array}$ & $\begin{array}{l}\text { Increased plasma levels and } \\
\text { increased side effects; for } \\
\text { tricyclic antidepressants, } \\
\text { potential increased risk for } \\
\text { cardiac conduction delay }\end{array}$ & None & \\
\hline
\end{tabular}


Drugs of abuse can also cause drug-drug interactions. MDMA, an amphetamine derivative also known as "ecstasy" that is primarily metabolized via the cytochrome P450 isoenzyme 2D6, reportedly led to a lethal overdose when taken by a patient being treated with ritonavir (283). Other drugs of abuse, particularly those metabolized via the cytochrome P450 isoenzymes 2D6 or $3 \mathrm{~A}$ (e.g., amphetamines, ketamine, heroin, cocaine, and $\gamma$-hydroxybutyrate) may lead to toxic events when taken by patients being treated with protease inhibitors.

Although some drug-drug interactions may be only theoretical, it is prudent for clinicians to explain these concerns during the informed consent process of psychotropic prescribing. Because of the extent of comorbid substance abuse among some HIV patients, a general warning of the possible interactions of combining antiretroviral agents, psychotropic medications, and recreational drugs is often indicated. Web sites are available to clinicians and patients that address antiretroviral drug interactions and may be useful educational and reference tools (www. aegis.com; www.drug-interactions.com; and www.dml.georgetown.edu/depts/pharmacology/ davetab.html).

Psychiatrists should remember that HIV patients who have been clinically stable on regimens of maintenance psychotropic medications and who later initiate combination antiretroviral therapies may require adjustments or changes in their psychotropic regimen. The rational choice of psychotropic medications must also include critical considerations of adherence. Generally, it is important to minimize the number of drug doses and to tie dosing to times of the day with natural cues (e.g., meals, work schedule, bedtime). Outlined here are unique factors to consider in administering specific psychotropic agents to HIV patients, particularly those patients simultaneously receiving antiretroviral therapies.

\section{Antidepressant agents}

The prevalence of mood disorders in HIV patients is higher than general population rates; evidence from effectiveness studies and clinical trials indicates that antidepressants are generally well tolerated by HIV patients, even those with symptomatic HIV infection or AIDS (284290). Thus, antidepressants are commonly prescribed to HIV patients for the treatment of depression as well as for other HIV-related conditions, such as chronic pain (e.g., peripheral neuropathy) (291). However, clinicians must be cautioned that two factors may limit the generalizability of some antidepressant studies. First, many studies of antidepressants in HIV patients were conducted before the availability of combination antiretroviral therapy and thus did not take into account possible pharmacokinetic complications (292). Second, a minority of published psychopharmacology studies has included patients with advanced stages of HIV infection or AIDS. There is, to date, no evidence to suggest adverse effects of antidepressants on the immune system (293).

\section{a) SSRIs}

Several studies have indicated that tricyclic antidepressants are less well tolerated than SSRIs $(287,294)$, leading some to recommend SSRIs over tricyclic antidepressants (277). One study found that despite efficacy for treating depression, fluvoxamine was poorly tolerated by HIV patients (295). Because similar metabolic pathways, particularly the cytochrome P450 isoenzyme 2D6, are utilized by SSRIs and ritonavir, the psychiatrist should maintain communication with other clinicians treating the patient. Although SSRIs are generally well tolerated across a broad range of blood levels, the untoward outcome of serotonin syndrome may be possible in some cases in which the cytochrome P450 isoenzyme 2D6 is inhibited (296). While the SSRIs themselves inhibit this isoenzyme, the potency of their inhibition is less than that of ritonavir. However, the potential impact of SSRI-related inhibition of the cytochrome P450 isoenzyme $2 \mathrm{D} 6$ should be remembered when coadministering SSRIs with other agents that are substrates of the cytochrome P450 2D6 isoenzyme. 


\section{b) Tricyclic antidepressants}

While tricyclic antidepressants are metabolized by other cytochromes, they rely principally on the cytochrome P450 isoenzyme 2D6 for clearance. Therefore if tricyclic antidepressants are coadministered with ritonavir, monitoring of ECG results and tricyclic antidepressant plasma levels is recommended because of the possibility of increased tricyclic antidepressant plasma levels from cytochrome $\mathrm{P} 450$ inhibition and associated tricyclic antidepressant side effects (e.g., delayed cardiac conduction, anticholinergic effects, orthostasis).

\section{c) Other antidepressants}

Nefazodone and the protease inhibitors are both potent inhibitors of the cytochrome P450 isoenzyme $3 \mathrm{~A}$, and their combination may be problematic. Nefazodone administration may theoretically decrease the metabolism of some protease inhibitors, leading to increased protease inhibitor plasma levels and increased side effects. However, one study that examined the efficacy of nefazodone in HIV patients found that only one of 15 patients had a clinically significant drug interaction that was related to coadministration with ritonavir (288). Of general concern is the coadministration of nefazodone or protease inhibitors with the antipsychotic pimozide, which can cause cardiac arrhythmias at increased plasma levels (296). Other potentially dangerous interactions with inhibitors of the cytochrome P450 isoenzyme 3A include the coadministration of most benzodiazepines (respiratory depression), clozapine (seizures), ergot alkaloids (sustained systemic vasoconstriction), and sildenafil (priapism).

Venlafaxine has been reported to decrease the concentration of indinavir significantly even though a lack of such interaction was predicted based on each drug's pharmacokinetic parameters. At this time, venlafaxine should be avoided in those patients receiving indinavir, since decreases in concentration of protease inhibitors can affect treatment efficacy and increase the chances for viral resistance to develop (297).

Previously it was thought that bupropion was eliminated via the cytochrome P450 isoenzyme 2D6. However, recent data have shown that it is actually cleared via the $2 \mathrm{~B} 6$ isoenzyme. Therefore, bupropion has been removed from the list of drugs contraindicated with ritonavir, and this is reflected in the revised product monograph.

No adverse events have been reported with the agents citalopram and mirtazapine. Citalopram has minimal inhibitory actions on the cytochrome system and may be particularly useful for patients taking multiple medications where drug-drug interactions are an ongoing concern. Mirtazapine, because of its sedating profile, may be particularly useful for depressed patients with insomnia. It may also be helpful for patients with poor appetite because of its tendency to increase appetite and also helpful for patients who may be prone to sexual dysfunction with other antidepressant agents.

\section{Antipsychotic medications}

Generally, the use of antipsychotic medication among patients with advanced HIV infection is associated with an increased incidence of extrapyramidal side effects. High-potency standard neuroleptics (haloperidol is the most commonly used in published reports) can be associated with severe dystonia, rigidity, akathisia, and parkinsonism (31, 298-301). Low-potency standard neuroleptics can also result in severe extrapyramidal side effects but overall appear to be less problematic than high-potency neuroleptics (302-305).

A study that contrasts with the perspective that standard neuroleptics are associated with severe extrapyramidal side effects in advanced HIV infection was conducted in hospitalized patients with AIDS who were randomly assigned pharmacologic treatment for delirium; 13 received chlorpromazine, and 11 received haloperidol (46). The study stated that "no clinically significant medication-related side effects were noted." However, patients were treated with very low doses of neuroleptic (i.e., average maintenance doses were $36 \mathrm{mg} /$ day for chlorpromazine and $1.4 \mathrm{mg} /$ day for haloperidol). The study is consistent with the rest of the literature in 
stressing the importance of treating patients with advanced HIV infection with the lowest possible effective dose when using a typical neuroleptic.

Neuroleptic malignant syndrome has been noted with standard neuroleptics in five reports. In one case, neuroleptic malignant syndrome occurred within 24 hours; in the four other cases, neuroleptic malignant syndrome occurred within days of initiating treatment (300, 306-309). All patients recovered with usual management. Onset of tardive dyskinesia has been reported within a period as short as 6 weeks or within months $(31,310,311)$. Confusion has also been reported with standard neuroleptics (312).

Most patients with extrapyramidal side effects respond to typical treatments, but one case report of a patient with parkinsonian side effects noted a slow response (299). Another patient had no response to multiple antiparkinsonian agents (313). Scurlock et al. (298) reported an AIDS patient who developed a grand mal seizure while taking chlorpromazine, and Jones et al. (314) reported a grand mal seizure in one man with symptomatic HIV infection who was taking amitriptyline and chlorpromazine.

Atypical antipsychotic agents appear to be better tolerated with fewer serious side effects, as evidenced by two case series of 30 combined patients who received risperidone $(310,315)$, one report of four patients who received remoxipride (298), and another of four patients who received molindone (312). Some of these patients had previously experienced severe side effects while taking typical neuroleptics. All were able to tolerate the atypical agents, and most but not all patients had a good therapeutic response. The most common side effects seen with risperidone were drowsiness and drooling (315). Extensive clinical experience suggests that olanzapine is also well tolerated for the treatment of psychosis, delirium, and behavioral disturbances among HIV patients.

It is of interest that movement disorders, including dystonia and parkinsonism, have been reported in advanced HIV infection without exposure to neuroleptics $(316,317)$. In the one case report of irreversible extrapyramidal side effects in a man with HIV-associated dementia following exposure to standard neuroleptics, an autopsy revealed numerous microscopic changes, including neuronal loss in the basal ganglia (313). It may be that damage to the basal ganglia is a factor that increases the susceptibility of HIV patients to extrapyramidal side effects after neuroleptic administration (318). A similar pattern of extrapyramidal side effect susceptibility in concert with neuronal damage has been described in the elderly $(311,319)$.

For all antipsychotic agents, the possibility of drug-drug interactions must be considered for those patients also taking antiretroviral medication. Antipsychotic dosage adjustments may be required to minimize side effects such as extrapyramidal side effects. Because of the rare doserelated side effect of agranulocytosis among patients taking clozapine, this particular agent should be monitored closely if given to any HIV patient (320). Likewise, clinicians should monitor for risk of seizures if clozapine is administered with ritonavir, since ritonavir inhibits the cytochrome P450 isoenzyme 2D6, clozapine's primary metabolic pathway. Pimozide should also be used cautiously in combination with any inhibitor of the cytochrome P 450 isoenzyme 3A because of risk of fatal arrhythmias at elevated doses. Depot antipsychotic medication should be avoided in advanced HIV disease.

\section{Psychostimulants}

Psychostimulants, such as methylphenidate and dextroamphetamine, are frequently used in HIV patients with neurocognitive impairment, fatigue, and, to a lesser extent, in some patients with depression $(321,322)$. These drugs are generally safe and well tolerated with no drug-drug interactions reported to date among HIV patients. The possibility of dopamine agonism inducing psychosis should be considered in vulnerable patients, such as those with a history of HIV-related CNS complications or a history of psychosis. Psychostimulants should be avoided or used judiciously among patients with a past or current history of amphetamine abuse. 


\section{Mood stabilizers}

\section{a) Valproate}

Use of valproate in patients with bipolar disorder is not uncommon, particularly among HIV patients who develop mania secondary to HIV-related complications or psychoactive substance use. Concerns have been raised about the potential for valproate to possibly accelerate disease progression in HIV-positive patients. This concern is based on the fact that valproate has been shown in vitro to lower glutathione levels, which leads to activation of HIV replication (323). However, a retrospective review by Maggi and Halman (324) of 11 patients with HIV infection who were also treated with valproate found no evidence of increased viral load in those receiving adequate antiretroviral therapy.

The metabolic pathways of valproate are not fully delineated, but this agent has been associated with hepatotoxicity. There is a case report of valproic acid-induced hepatotoxicity when valproate was used together with ritonavir and nevirapine (325). Valproate inhibits glucuronyl transferase. Zidovudine uses glucuronyl transferase as its primary pathway of metabolic clearance. Therefore, increased plasma levels of zidovudine and associated side effects could result when the two agents are combined.

Generally, divalproex sodium is better tolerated than valproic acid, since it produces fewer gastrointestinal side effects and can be dosed less frequently (two or three times a day rather than four), particularly among HIV patients who may have preexisting gastrointestinal illness. Increased side effects may be caused by increased concentrations of free valproate in HIV patients because of factors such as the reduction of plasma protein levels, the increased percentage of free drug due to chronic illness, or drug-drug interactions that may decrease protein binding of valproate (326).

\section{b) Lithium carbonate}

Lithium is commonly used in HIV patients with primary bipolar disorder. Because it does not require hepatic metabolism, it is a rational choice for many patients taking antiretroviral agents who require extensive hepatic metabolism. Lithium should be used with great caution in patients who develop HIV-related nephropathy, a generally irreversible complication that can lead to decreased lithium clearance and possible lithium toxicity. When given at doses necessary to maintain serum lithium concentrations between 0.5 and $1.5 \mathrm{meq} / \mathrm{liter}$, lithium has been associated with signs of toxicity in some HIV patients, even among patients without renal disease (327). Thus, clinical experience suggests that lithium is best avoided in patients with probable AIDS mania or advanced HIV disease due to risk of toxicity.

\section{c) Carbamazepine}

Although not absolutely contraindicated, carbamazepine is used less commonly in HIV patients because of its potential to cause bone marrow complications (e.g., leukopenia or aplastic anemia). This risk should be avoided in immunocompromised patients. Carbamazepine, like other anticonvulsants, phenobarbital, and phenytoin, is known to induce activity of the cytochrome P450 isoenzyme 3A. This induction can accelerate antiretroviral drug metabolism and possibly cause decreased plasma levels and diminished efficacy. It is recommended that carbamazepine concentrations be closely monitored.

\section{d) Newer anticonvulsants}

Lamotrigine, gabapentin, and topiramate are newer anticonvulsants that have recently been used off-label to treat selected populations of patients with bipolar disorder, typically those with rapid cycling, mixed mood states, or bipolar depression. While no data currently support their use for mood disorders in HIV patients, gabapentin may offer some advantages over other anticonvulsants because it is neither protein bound nor metabolized by the liver. Gabapentin 
is thus less likely than other anticonvulsants to lead to drug-drug interactions. Gabapentin has been successfully used in HIV patients to treat peripheral neuropathy, which can be both a complication of HIV disease and a side effect of some antiretroviral agents.

\section{Anxiolytic and sedative-hypnotic medications}

\section{a) Buspirone}

Buspirone is commonly used and generally well tolerated in HIV patients as a treatment for generalized anxiety disorder (328). There are no reports of HIV-related drug-drug interactions with buspirone. However, one case report describes induction of manic psychosis following a single dose of buspirone in a patient with asymptomatic HIV illness (329).

\section{b) Benzodiazepines}

Benzodiazepines are commonly used in HIV patients and are best used for short periods to minimize the risk of tolerance or addiction and to maximize effectiveness. This class of drugs is generally best avoided in patients with alcoholism and those with a history of substance abuse. Benzodiazepines are metabolized primarily by the cytochrome P450 isoenzyme 3A; however, oxazepam, lorazepam, and temazepam are metabolized by direct conjugation via glucuronyl transferase, resulting in shorter elimination half-lives and less likelihood of metabolite accumulation and associated side effects.

When some benzodiazepines are coadministered with inhibitors of the cytochrome P450 isoenzyme 3A (such as protease inhibitors), one would expect a decrease in benzodiazepine clearance with increased sedation, potentially resulting in respiratory depression. Even saquinavir's relatively low-potency inhibition of the cytochrome P450 isoenzyme $3 \mathrm{~A}$ has been shown to prolong the sedative effect of midazolam (282). Although one study that examined the chronic effect of ritonavir administration on alprazolam metabolism reported a $10 \%-15 \%$ decrease in expected alprazolam levels (330), a more recent study found that short-term, lowdose ritonavir administration significantly impaired alprazolam clearance (331). These studies call attention to the need for judicious use of alprazolam in combination with ritonavir.

An additional consideration when using benzodiazepines that are metabolized by glucuronidation (e.g., oxazepam, lorazepam, temazepam) involves ritonavir's inductive effect on glucuronyl transferase activity (332). The concentration of glucuronyl transferase substrates may be decreased when these agents are coadministered with ritonavir. Therefore in some cases, patients taking one of these three benzodiazepines may require higher doses for symptom control. Benzodiazepinedependent patients may also require higher doses to avoid the onset of withdrawal (296).

\section{Medications for HIV-related wasting}

Some patients with wasting syndrome can be helped by medications that serve as appetite stimulants to combat loss of appetite and associated weight loss. Four agents currently have been approved by the Food and Drug Administration (FDA). Two of these agents, dronabinol and oxandrolone, are Schedule II controlled substances. Because these agents have unique psychiatric side effect profiles, they should be monitored closely among patients who receive psychiatric care.

Dronabinol is a cannabinoid indicated for HIV-associated anorexia and nausea. It is a major active metabolite in marijuana and, like marijuana, has complex effects on the CNS, including sympathomimetic activity. Oxandrolone is an anabolic steroid synthetically derived from testosterone that is used for weight gain and promotion of lean body mass (333-335). Its side effect profile is similar to that of other anabolic steroids, including mood effects. Another agent, megestrol acetate, is a synthetic derivative of progesterone that increases fat rather than muscle gain. It also lowers testosterone and may produce side effects (e.g., mood disturbances) similar to those associated with glucocorticoids. Somatotropin is an injectable form of recombinant human growth hormone indicated for the treatment of AIDS wasting and cachexia (336-340). It 
is an anabolic and anticatabolic treatment that combats the loss of lean body mass and is generally well tolerated by patients. The effects of somatotropin are often lost when it is discontinued.

These medications can be extremely beneficial for patients with significant anorexia, weight loss, and nausea. However, because they are usually used in more advanced stages of HIV disease, when patients are more medically frail and receiving multiple other medications, psychiatrists should actively monitor response, side effects, and drug interactions. Limited data do not indicate a high incidence of psychiatric side effects.

Thalidomide, a potent teratogen once marketed as a sedative and morning sickness agent in the 1950s and 1960s, is currently available on a restricted basis for HIV patients for the treatment of wasting syndrome $(341,342)$. The agent is FDA-approved as an immunomodulatory drug for the treatment of severe HIV-related oral ulcers. Prescribing physicians must register under a special restricted distribution program with the FDA. Primary psychiatric side effects include CNS depression; few data are available concerning drug-drug interactions, although thalidomide does not appear to be metabolized by the liver to any large extent.

\section{Testosterone}

A number of clinical trials have found testosterone to be effective in the treatment of a number of HIV-related symptoms (e.g., fatigue, wasting, impaired sexual functioning, and mild depression) often associated with more advanced HIV disease (126, 343, 344). Although typically used to treat hypogonadism in the general population, clinical response to testosterone in HIV patients is not correlated with serum testosterone levels at baseline. HIV-infected men with normal levels of testosterone respond as well as those with lower than normal levels (126). The most common psychiatric side effects of testosterone are changes in mood and irritability.

\section{Opiate analgesics}

Many HIV patients are treated with opiate analgesics for both acute pain syndromes, such as that associated with Candida esophagitis, as well as chronic pain conditions (e.g., neuropathic and myopathic pain syndromes). Chronic pain is also common in advanced HIV disease, particularly among cachectic patients with wasting syndrome. Because opioids are metabolized primarily via the cytochrome $\mathrm{P} 450$ isoenzymes $2 \mathrm{D} 6$ and $3 \mathrm{~A}$, they should be used cautiously in conjunction with antiretroviral agents that are likely to increase their plasma levels.

Some opioids, such as codeine and hydrocodone (as well as the nonopiate tramadol), must be converted into active metabolites to produce analgesic effects. This conversion can be blocked by cytochrome $\mathrm{P} 450$ inhibitors, which results in reduced pain control in addition to adverse reactions from the buildup of unmetabolized drug (345). Oxycodone and morphine, which are primarily glucuronyl transferase substrates, are alternatives for pain control. However, in the presence of a glucuronyl transferase inducer such as ritonavir, their analgesic effect can be diminished as well $(296,332)$. As HIV disease progresses, the provision of safe, effective pain control requires clear understanding of the above factors.

\section{Drugs used in the treatment of substance use disorders}

\section{a) Opioid dependence}

There is limited information available on the extent and clinical implications of drug interactions in HIV-positive patients with comorbid substance use disorders. However, several studies have been published. Methadone has been shown to significantly increase zidovudine levels, but zidovudine has not been shown to affect methadone levels (346-348). Interim results from a study of the interaction of zidovudine with LAAM, buprenorphine, or naltrexone indicate that LAAM does not affect zidovudine levels, whereas buprenorphine may decrease levels nonsignificantly (349). Another report indicates that abacavir, a newer nucleoside analogue reverse transcriptase inhibitor, increases methadone clearance, although the proposed mechanism re- 
mains unclear (350). Preclinical studies of the coadministration of protease inhibitors and methadone revealed that ritonavir causes a twofold increase in the area under the curve for methadone level and that indinavir causes a 30\% increase, compared to no increase with saquinavir (351). One case report has indicated that nelfinavir may induce cytochrome P450 enzymes leading to decreased methadone levels (352). Nevirapine and efavirenz induce cytochrome $\mathrm{P} 450$ enzymes important to methadone metabolism and are reported to precipitate opiate withdrawal symptoms in methadone maintenance patients $(278,353)$. The consequences of undetected drug interactions among opioid-dependent patients are complicated and include nonadherence with treatment regimens, the potential development of resistant HIV strains, increased illicit drug abuse, drug toxicity, and loss of efficacy. In administering methadone to HIV patients taking protease inhibitors or nonnucleoside reverse transcriptase inhibitors, clinicians should adjust doses to compensate for cytochrome P450 enzyme effects.

\section{b) Alcohol dependence}

There is no clinical information regarding the use of disulfiram in patients with HIV infection. Psychiatrists should therefore follow general guidelines for the use of disulfiram cautiously. Naltrexone, an opioid antagonist, is indicated in the treatment of alcohol dependence and for the blockage of the effects of exogenously administered opioids. Because naltrexone can cause hepatocellular damage when given in excessive doses, it should be used cautiously in patients with liver disease. It should also be noted that naltrexone's opioid antagonism might block the clinical effects of some commonly used medications in HIV patients that contain opioids (e.g., antidiarrheal medications, cough and cold preparations).

\section{Alternative/complementary agents}

Patients with HIV infection may use a wide range of alternative/complementary agents for preventive and symptomatic treatment of HIV-related complications. Of particular interest to psychiatrists are those agents used to treat psychiatric symptoms. For example, patients may take Hypericum perforatum (St. John's wort) or the nutritional supplement $S$-adenosylmethionine (or SAM-e) to treat depression, kava kava for anxiety, melatonin or valerian root for insomnia, and ginkgo biloba for memory problems. One published report found that St. John's wort, which contains an inducer of the cytochrome P450 isoenzyme 3A, dramatically lowered levels of indinavir (and presumably other substrates of the cytochrome P450 isoenzyme 3A) (354). The FDA thus issued a public health advisory that concomitant use of St. John's wort with protease inhibitors and nonnucleoside reverse transcriptase inhibitors is not recommended. There are no data regarding other alternative/complementary agents at this time.

\section{PSYCHOSOCIAL TREATMENT}

Since the beginning of the AIDS epidemic, psychosocial support has emerged as a cornerstone of the comprehensive treatment for many persons infected and affected by HIV/AIDS. For many HIV patients, psychotherapy and psychosocial interventions have been invaluable in the search for meaning during the course of living with HIV (355). Psychotherapy can be an important intervention to address conditions that may interfere with a patient's acceptance of HIV illness or their ability to work cooperatively with their health care team. With the advent of new antiretroviral treatments, new psychotherapy themes have emerged, including changes in role definitions and life trajectory as well as treatment adherence challenges (356-358).

For patients who are socially disenfranchised, psychosocial treatments may consist of basic services that assist them in gaining access to food, shelter, transportation, and child careessential elements of a care plan that diminish health care delivery barriers and promote mental health care utilization. 
Generally, psychosocial treatments are those treatments, often offered in the community, that provide direct assistance along such parameters as social adjustment, social support, coping, and overall adjustment to HIV infection. Psychosocial interventions often occur in group formats, such as informal support groups, although more formal groups may tailor their membership (i.e., women, men, mothers, caregivers) or their mission (HIV risk reduction, treatment adherence) $(359,360)$. Other formats include peer counseling, a supportive intervention typically offered by HIV-positive consumers based in community AIDS service organizations.

Other psychosocial treatments have been developed that incorporate a service model (e.g., psychosocial rehabilitation programs for persons with HIV/AIDS, day treatment programs for HIV-positive persons with neurocognitive impairment) or a service theme (e.g., permanency planning for parents with advanced AIDS, HIV prevention, case management, disclosure of HIV status) $(67,79,355,361)$.

Researchers have described a wide range of psychological and psychosocial sequelae from HIV/AIDS (251). In their review of empirical findings, Kalichman and Sikkema (362) showed that psychological complications include distress, anger, guilt, bereavement, and the full spectrum of psychiatric disorders. Syndromes such as depression may appear, subside, and recur during the course of living with HIV/AIDS. Moreover, certain psychosocial conditions, such as increased social support and fewer incidents of HIV-related discrimination, have been shown to predict greater life satisfaction among HIV patients (143).

Psychosocial treatment of HIV-related complications will often involve referral of patients to community AIDS service organizations that specialize in the provision of psychosocial support. While many of the treatment issues are also themes that will emerge in psychotherapy (i.e., stigma, coping strategies, or disclosure of HIV status), support groups and other interventions provide a model for structuring social support over the course of living with HIV infection (362). Psychosocial services may take on added importance for patients living in rural regions, in which more community HIV-related stigma has been documented (143).

Psychosocial interventions have been shown to enhance adaptive coping strategies, social support, perceived sense of meaning and purpose, and self-esteem and also have been shown to lower anxiety and HIV risk behaviors among HIV-positive adults $(359,363-365)$. Because of the spectrum of social, psychological, and neuropsychiatric consequences of HIV/AIDS, psychiatric treatment should consider the relevance for a given individual of a wide variety of potential psychosocial interventions, spanning the spectrum from individual to family or group psychotherapy as well as psychodynamic/psychoanalytic, interpersonal, behavioral, or supportive approaches.

\section{LEGAL AND ETHICAL ISSUES}

Psychiatrists caring for HIV-infected patients can be faced with complex legal and ethical issues, some of which are unique to HIV infection. The AIDS Litigation Project conducted a national review of cases that involved individuals with HIV/AIDS in the federal and state courts in the United States between 1991 and 1997. The review identified important subsets of litigation, which included issues of "testing and reporting; privacy, the duty to warn, and the right to know; physician standards of care in prevention and treatment; and discrimination and access to health care" (366).

Most U.S. states have legal requirements surrounding HIV testing that require written informed consent and a minimum amount of pre- and posttest counseling-both to those who test positive and to those who test negative. Furthermore, many states are required to report positive HIV test results to public health agencies. Some states mandate that the report include the name of the individual who tested HIV positive. All states have mandatory name reporting when a patient is diagnosed with an AIDS-defining condition.

For those infected with HIV, confidentiality of HIV status is often paramount, especially for patients who are asymptomatic or who are concerned about possible discrimination by their em- 
ployers or by insurance companies. Psychiatrists are typically attuned to confidentiality issues and regard patient-doctor confidentiality as fundamental to effective treatment. Yet, there are limits to confidentiality, such as when there is potential for others to be harmed, and these limitations should be discussed with patients when treatment is initiated.

For persons infected with HIV, particularly those recently diagnosed, their recent sexual partners and/or those with whom they share injection equipment should be informed of their potential exposure to HIV. These partners can benefit from HIV counseling and testing and knowing their HIV infection status. Many health departments offer services known either as "partner notification" or prevention counseling and referral services (367-369).

Another important issue involves the dangerousness of HIV patients. A patient with HIV infection is not a danger to others in the absence of imminent threats to harm others. In general, there are no legal requirements for physicians to inform partners of HIV-infected patients of their HIV status, especially if the patient agrees to notify their partner or if they are clearly practicing "safer sex." However, because HIV-infected patients are able to transmit the virus and potentially put others in danger of infection, psychiatrists who care for HIV patients have an ethical requirement to counsel their patients about modes of HIV transmission and promote safer behavior, such as the consistent use of condoms and/or the use of clean needles. Psychiatrists can offer to assist patients in notifying individuals at risk or help refer a patient to public health officials for partner notification, if necessary.

On the other hand, if a patient has not disclosed their HIV-positive status to a partner and continues high-risk behavior, psychiatrists may have a duty to warn identifiable sexual partners or other at-risk individuals. APA policy reads that, "If a patient refuses to change behavior that places others at risk for HIV infection or to inform individuals at ongoing risk, or if the psychiatrist has good reason to believe that the patient has failed to or is unable to cease such behaviors or to inform those at risk, it is ethically permissible for the psychiatrist to notify identifiable individuals at risk or to arrange for public health authorities to do so" (370). Psychiatrists need to be mindful that while it may be ethically permissible to notify, it may not be legally permissible, and therefore psychiatrists should obtain legal advice before breaching confidentiality.

For patients who are impulsive or psychotic, protective measures such as voluntary or involuntary psychiatric hospitalization are options for treatment of psychiatric disorders and protection of others. For antisocial individuals, hospitalization should not be used merely as a means of social control.

During the course of HIV illness, and especially after the onset of AIDS, patients may experience cognitive impairment or symptoms of dementia. Such disturbances may affect a patient's ability to give informed consent to accept or refuse treatment. When psychiatrists are asked to conduct competency evaluations for patients with HIV/AIDS, a comprehensive mental status examination that includes an evaluation of cognition is critical, followed-if indicated — by formal neuropsychological testing and assessment of specific treatment decision-making capacity.

The opposite of a patient's refusal of treatment is the refusal on the part of physicians to provide HIV treatment to psychiatric patients. Because antiretroviral therapies involve complex medication regimens and adherence to treatment is critical, physicians may be biased against prescribing these treatments to patients with a comorbid mental illness such as a substance use disorder. While refusal of treatment due to poor adherence is appropriate, refusal of treatment solely on the grounds of a mental illness is not. When the psychiatrist is a consultant who does not intervene directly in the care of the patient, he or she will need to work with the primary clinician to help ensure that appropriate psychiatric treatment is received (371).

\section{E. ADHERENCE TO TREATMENT}

Potent antiretroviral treatment has given hope to patients infected with HIV yet simultaneously has increased the complexities of managing HIV illness (372). For instance, the combination 
of three or more medications used in most antiretroviral treatment regimens makes adherence difficult. In addition, some medications must be taken with food, others without food; dosing is usually twice a day but sometimes can be more frequent. Side effects can be significant, requiring discontinuation or the initiation of a new combination of medications. Because antiretroviral treatment should begin as an aggressive broad attack on HIV replication, doses of the drugs are generally not titrated upward but rather are initiated at the full dose. Thus, patients may experience many initiation side effects.

Adherence to antiretroviral therapy regimens is critical, as evidenced by a study that measured adherence by using a microelectronic monitoring system (95). The authors found that 95\% adherence to treatment was associated with complete viral suppression but that failure rates increased sharply with less than $95 \%$ adherence. Researchers have documented that patients who receive potent antiretroviral treatment but who are not enrolled in clinical trials show viral suppression rates one-half those of study patients, which suggests the real-world difficulty of maintaining strict adherence (373).

Adherence to any kind of medical treatment is a well-established problem. Overall adherence rates vary from $20 \%$ to $80 \%$ and average $50 \%$ (374). Psychiatrists should make every effort to treat and control psychiatric disorders that affect a patient's ability to maintain close adherence to an HIV treatment regimen. Each year, about 7\% of patients with AIDS develop HIV-associated dementia (375). This condition can interfere with ability to follow and understand treatment regimens. In addition, researchers have found that depression can be a cause of nonadherence and that treatment of depression might thus improve medical outcomes (96). Strategies that have proven useful in improving adherence for both psychotropic and antiretroviral medication have been reported and are summarized below (376).

\section{Institutional factors}

How a health care system is structured will strongly influence whether clinicians and patients can collaborate and enhance adherence. One important element is a respectful and culturally sensitive environment. The availability and continuity of clinicians over time with 24 -hour/day phone coverage to handle crises that might disrupt a regimen (severe side effects, loss of medication), as well as clinician caseloads that permit time for adequate discussion with patients, all promote adherence. Hospitals do not always dispense medications on the 8- or 12-hour schedules required for antiretroviral dosing. Last, social service mechanisms such as case management for handling financial and logistical barriers to obtaining and following the regimens provide crucial support.

\section{Physician factors}

When initiating antiretroviral treatment, it is essential for the physician to prepare the patient. Because the best chance at viral suppression occurs with the first regimen, it makes sense to wait until the patient is ready. For a psychiatrist, it is important to treat or recommend treatment for comorbid alcohol/substance use disorders, depression, psychotic illnesses, or personality disorders that are likely to interfere with adherence (377). At the same time, if the patient is motivated to begin antiretroviral treatment, this can be a good opportunity to introduce psychiatric or substance use treatment if indicated.

\section{Clinical strategies}

Multiple approaches to enhancing adherence are often needed (Table 17). For longer-term adherence, individual medication management, in which a staff member provides assessment and problem-solving support, may improve adherence (378). 
TABLE 17. Interventions to Increase Patient Adherence to Antiretroviral Regimens

Prepare patients

Discuss use of medications before prescribing

Outline pros and cons of therapy

Acknowledge commitment required, consequences of noncompliance, and

benefits of therapy

Provide written instructions

Inform patients of expectations, including side effects

Provide information on who patients should call if significant side effects occur

Schedule a follow-up appointment soon after initiating therapy

Review importance of therapy

Inform patients that they must continue to take all medications

Review the effects of stopping one medication

Outline procedure for obtaining refills

\section{Recognize patient lifestyle and preferences}

Twice-daily dosing benefits may outweigh initial side effects; ritonavir may be preferred

Consider whether patients prefer tolerability over convenience; nelfinavir or indinavir may

be preferred

Discuss midday dosing

Recommend medication timers or calendar

Help patients plan for away-from-home dosing

Simplify regimens

If possible, prioritize or eliminate medications when patients are overwhelmed

Look for and address nonadherence

Consider regimens that minimize cross-resistance

Use regimens that leave options for future effective antiretroviral therapy

Inquire about adherence

Inquire about medication-taking behavior at each visit

Anticipate relapses in adherence, even after long-term use of medication

\section{F. SPECIFIC SYNDROMES}

There are numerous psychiatric difficulties that can occur in the context of HIV infection, yet studies of the treatment of HIV-related psychiatric disorders are limited. This section summarizes the existing scientific literature on the treatment of specific psychiatric disorders for patients with concurrent HIV/AIDS.

\section{Dementia and the spectrum of cognitive disorders}

Most research studies on the treatment of cognitive disorders associated with HIV infection have focused on HIV-associated dementia. Fewer studies have looked at the benefits of intervention at earlier stages of cognitive decline despite the possibilities for treating disorders, such as minor cognitive motor disorder, that involve neuronal cell dysfunction rather than cell death. Treatment of neuropsychological impairment without clinical disorder has been studied to some extent, and protection against neuropsychological impairment has been studied least.

Pharmacologic treatment strategies for cognitive disorders can be divided into four types: 1) antiretroviral therapies, 2) therapies aimed at immunological measures or inflammatory mediators, 3) therapies aimed at bolstering the response of the brain to the onslaught of the infection (e.g., neurotransmitter manipulation), and 4) nutritional therapies. Most controlled studies have investigated the efficacy of antiretroviral therapies, and while these studies have advanced our knowledge about interventions for cognitive disorders, several key factors must 
be kept in mind. First is the fact that most published studies to date report on the treatment strategy of administering a single antiretroviral agent. Their findings are therefore difficult to interpret in light of the multidrug regimens that are now the standard of care in developed countries. Second, the reports vary widely with regard to the study population, since some studies enrolled subjects on the basis of established criteria for HIV-associated dementia or minor cognitive motor disorder but other studies enrolled cognitively impaired subjects without specifying whether they also met criteria for a clinical disorder. Third, the range of HIV clinical severity also varied widely in study subjects.

Whether antiretroviral agents penetrate the blood-brain barrier sufficiently to adequately suppress viral replication is a key issue that requires further study $(379,380)$. We know that nearperfect adherence to antiretroviral therapy is required to maintain plasma viral load at undetectable levels. Even in the best-case scenario, if decreased plasma viral load is achieved and levels of antiretroviral agents in CSF or brain tissue are optimized, it is not yet known whether complete suppression of replication in the CNS can be achieved (381). Another theoretical concern is that if antiretroviral resistance develops in the CNS, it is possible that resistant HIV could then reseed the peripheral circulation. This mechanism could potentially lead to increased prevalence of neurocognitive disorders as well as systemic progression of HIV disease.

\section{a) Zidovudine treatment of cognitive disorders or dysfunction}

Two randomized, placebo-controlled clinical trials have provided evidence for the effectiveness of zidovudine in reducing neuropsychological impairment for patients with HIV infection. Sidtis and colleagues (382) treated a group of 40 homosexual men (early-stage dementia and mean CD 4 cell counts of 500 cells $/ \mathrm{mm}^{3}$ ) with 1000 or $2000 \mathrm{mg} /$ day of zidovudine or placebo. They found that both treatment groups demonstrated significant combined mean neuropsychological z score improvements when compared to the placebo group. In another randomized, placebo-controlled clinical trial, Schmitt and colleagues (383) used zidovudine to treat 281 subjects who did not necessarily have clear-cut signs of neuropsychological impairment or cognitive disorder but were stratified into two groups, AIDS or early asymptomatic HIV infection, on the basis of CD 4 cell count. Significant improvements were found in attention, memory, visualmotor, and simple motor function at 4 months in both groups, which led to premature discontinuation of the study.

An open study of zidovudine in injection drug users found improvements in neuropsychological functioning on a number of subtests (384). Four case control studies retrospectively examined whether zidovudine treatment was associated with outcomes such as reduced leukoencephalopathy $(385,386)$, lower CSF $\beta_{2}$-microglobulin levels (which are related to neuropsychological improvement) (387), or improved neuropsychological measures (388). Three of the four studies found that the zidovudine-treated patients had significantly positive findings compared to untreated patients.

While the above studies support the efficacy of zidovudine, they do not resolve the issue of optimal dosage nor zidovudine resistance (380). Doses of up to $2000 \mathrm{mg} /$ day of zidovudine were used in some of the above studies, which are often associated with significant toxicity, including neutropenia and anemia. A typical zidovudine dose used now is $500-600 \mathrm{mg} / \mathrm{day}$, and many patients cannot tolerate even these reduced doses (375). Patients can develop resistance to zidovudine, especially if used alone, and because zidovudine-resistant strains can be transmitted from one person to another, some HIV experts have considered the need for testing for zidovudine resistance before choosing which agents to use in treatment (389).

\section{b) Other antiretroviral treatments of cognitive disorders or dysfunction}

Treatment studies of antiretroviral agents other than zidovudine are fewer but illuminating. De Ronchi and colleagues (390) conducted a clinical trial $(\mathrm{N}=88)$ that compared zidovudine to didanosine to refusal of therapy in both symptomatic and asymptomatic HIV patients. They 
found that for subjects who were symptomatic for HIV disease, both zidovudine and didanosine treatment resulted in better performance on all neuropsychological subtests. In general, asymptomatic subjects did not show significant differences in neuropsychological performance compared to the untreated group. The findings suggest differences in effectiveness based on HIV clinical status.

A more recent placebo-controlled study involved the use of abacavir, a nucleoside analogue reverse transcriptase inhibitor with good CSF penetration, in 99 subjects with moderate to severe HIV-associated dementia (391). This study did not demonstrate significant differences in neuropsychological scores between abacavir and placebo groups, but a major limiting factor in this study design was that abacavir alone was added to an already failing antiretroviral treatment regimen.

Of currently available antiretroviral agents, the CSF penetration of stavudine is relatively high ( 0.4 ratio with serum), but a recent study indicated that stavudine nevertheless penetrates brain tissue poorly (392). The individual protease inhibitors have not been well studied for effects on neurocognitive impairment, and all but indinavir ( 0.16 ratio with serum) penetrate the CSF poorly (393). Regarding the nonnucleoside reverse transcriptase inhibitors, nevirapine has been shown to have a 0.45 CSF-to-serum ratio and therefore may be a good candidate to include in future controlled studies of HIV-associated cognitive disorders (381).

Ferrando and colleagues (394) studied the effects of various combination antiretroviral therapy regimens on measures of cognitive motor function. They found that tests of attention, concentration, psychomotor speed, learning, and memory were significantly better in those subjects taking antiretroviral therapy than in those who were untreated. Furthermore, they found that those without neuropsychological impairment had lower mean viral load levels, which suggests that antiretroviral treatment benefits neuropsychological functioning through lowering viral load. A similar conclusion was offered by Sacktor et al. (40).

\section{c) Antiretroviral treatments to prevent HIV-associated dementia}

A few studies have examined the use of antiretroviral medications to prevent HIV-associated dementia. Moore and colleagues (395) conducted a study of 863 homosexual men treated with zidovudine who were evaluated every 2 months for 2 years or until death. Development of HIVassociated dementia continued with zidovudine treatment but was significantly correlated with low CD4 cell count. Montforte and colleagues (396) used didanosine in a study of 1,047 subjects (median CD4 cell count of 47 cells $/ \mathrm{mm}^{3}$ and mean prior zidovudine treatment of 19 months). Subjects were followed every 2 months to a clinical endpoint (AIDS-defining condition, severe adverse event, or death). They reported a very low incidence of HIV-associated dementia (11 subjects, or 1\%), although no formal criteria for defining HIV-associated dementia were offered. One randomized clinical trial with 32 subjects that compared zidovudine treatment to placebo found no significant difference in rate of developing dementia by treatment assignment (397). HIV-associated dementia prevention requires further study, but it appears that treatment with antiretroviral medications may prevent the development of HIV-associated dementia and other cognitive motor disorders.

\section{d) Interventions that affect inflammatory mediators}

Although peptide T can be thought of as "antiretroviral," since it blocks the binding of envelope glycoprotein to CD4 receptors, it more likely exerts its effects through decrements in the deleterious effects of inflammatory mediators known as cytokines, such as tumor necrosis factor. A multisite, randomized, placebo-controlled clinical trial with intranasal peptide $T$ was conducted on a sample of 215 patients with HIV-associated neurocognitive impairment, most of whom were taking a single antiretroviral agent or a two-drug combination regimen (398). The study found no statistically significant differences between treatment and placebo groups on global neuropsychological z scores. However, subgroup analyses showed that subjects with a CD4 
count of 201-500 cells $/ \mathrm{mm}^{3}$ and subjects with at least moderate neuropsychological impairment at baseline improved significantly with peptide $T$ compared with those given placebo $(\mathrm{p}<0.05)$. In addition, peptide $\mathrm{T}$ was well tolerated with no clinically significant toxic effects.

Studies of inflammatory mediators are indicated because researchers have postulated that HIV-related neuronal injury involves the activation of voltage-dependent calcium channels and $N$-methyl-D-aspartate (NMDA) receptor-operated channels (399). Therefore, studies are underway using memantine (an NMDA antagonist), inhibitors of tumor necrosis factor- $\alpha$, thalidomide, pentoxifylline, and naloxone (an inhibitor of interferon- $\alpha$ ). Mapou and colleagues (400) conducted a clinical trial with interferon- $\alpha-n 3$ in 20 asymptomatic subjects with HIV infection and reported significant improvements in neuropsychological performance in selected subtests. While interferon has been associated with neuropsychological side effects, including depression, Skillman et al. (401) reported that such side effects are minimal.

Nimodipine is a voltage-dependent calcium channel antagonist that has been postulated to prevent HIV-related neuronal injury. Two controlled studies of nimodipine, one in combination with zidovudine (402) and one with nimodipine alone (403), found no significant differences in overall neuropsychological performance.

\section{e) Treatments involving neurotransmitter manipulation}

Stimulant drugs have been used as palliative agents to help manage symptoms of fatigue, decreased concentration, or memory deficits among patients with HIV-associated dementia or minor cognitive motor disorder. With regard to the specific effect of stimulants on neurocognitive impairment, there has been one randomized, placebo-controlled clinical trial of a small sample of eight opioid-dependent patients maintained on a regimen of methadone (404). This 7-day crossover study with sustained-release methylphenidate $(20-40 \mathrm{mg} /$ day) resulted in nonsignificant neuropsychological z score improvements from baseline with methylphenidate but not placebo. Fernandez and colleagues (321) presented case reports of treating patients with HIVrelated disease with methylphenidate (30-90 mg/day) or dextroamphetamine (30-60 mg/ day). All patients improved significantly; however, there was no placebo control group and the sample size was small. Similar data from a small trial are offered by Holmes et al. (405).

Studies of dopaminergic agonists are supported by preliminary data in the form of case studies of carbidopa and L-dopa (303). The Dana Consortium conducted a randomized, placebocontrolled clinical trial of 36 subjects with HIV-associated cognitive motor impairment who were given L-deprenyl, a putative antiapoptotic agent, and found that deprenyl-treated subjects showed an improvement in neuropsychological test performance, specifically in verbal memory (406). Investigation of the dopaminergic agent pramipexole is currently under way.

Other neurotransmitters such as serotonin and 5-hydroxyindoleacetic acid have been found to be decreased in CSF as HIV disease progresses (407). It is possible that increasing CNS serotonin levels through administration of agents such as SSRIs may be useful in the treatment of cognitive motor impairment.

\section{f) Nutritional therapies and HIV-associated cognitive disorders}

Nutritional therapies need to be considered as potential interventions for the cognitive motor symptoms associated with HIV infection. A case report of a subject with apparent late-stage HIVassociated dementia and a low cobalamin (vitamin $\mathrm{B}_{12}$ ) level due to decreased intrinsic factor secretion responded over 2 months to $B_{12}$ replacement therapy with complete symptom resolution (408). Oxidative free radical scavengers such as vitamin E, the experimental antioxidant OPC14117 , and the trace mineral and antioxidant selenium may prove to be therapeutically useful.

\section{Delirium}

Antipsychotic medications have been the treatment of choice in treating delirious states that develop in patients with HIV infection, with most evidence arising from case reports or uncon- 
trolled trials $(409,410)$. For patients with HIV infection, a prominent issue is the observed increased sensitivity to side effects $(299,300)$. This sensitivity applies to extrapyramidal side effects with higher-potency agents and also to cognitive side effects with lower-potency antipsychotics.

To date, only one randomized clinical trial has studied the treatment of delirium associated with HIV infection. Breitbart and colleagues (46) approached 244 hospitalized AIDS patients and monitored them for the development of delirium. Among the 30 subjects who went on to develop delirium, 310 episodes of delirium occurred that met both DSM-III-R and Delirium Rating Scale criteria (411). The 30 subjects were randomly assigned to receive haloperidol ( $\mathrm{N}=$ 11), chlorpromazine $(\mathrm{N}=13)$, or lorazepam $(\mathrm{N}=6)$. Because the six subjects taking lorazepam developed signs of toxicity (oversedation, disinhibition, ataxia, and increased confusion), this treatment was terminated prematurely. Both haloperidol and chlorpromazine treatment resulted in significantly reduced scores on the Delirium Rating Scale in the first 24 hours. The researchers did not report significant problems with extrapyramidal side effects in study patients, but mean daily doses were low.

Scattered case reports or series suggest that other antipsychotic agents may be useful for treating HIV-associated delirium. For instance, molindone (312), risperidone (315), and droperidol $(412,413)$ have been suggested as alternatives to the standard antipsychotic medication because of differences in side effect profile, onset of action, or potency.

\section{Mood disorders}

Disturbances of mood occur frequently in the context of HIV infection. Treatment studies are quite varied with regard to variables such as severity of mood disorder, methodology to categorize or diagnose mood disorders, outcome measures, and stage of HIV illness.

\section{a) Acute treatment of depressive disorders - somatic treatments}

Many studies, including randomized controlled trials, open-label trials, case series, and case reports, support the efficacy and safety of many antidepressants in the acute treatment of depressive disorders, including major depression, in a wide range of HIV-infected populations at all disease stages.

Wagner and colleagues (286) reported on a series of 6-week studies that used random assignment and placebo control to examine the efficacy of standard and alternative antidepressants for major depression in HIV-infected patients. The agents used at standard doses were fluoxetine, sertraline, imipramine, dextroamphetamine, and testosterone. Response rates to the different agents ranged from $70 \%$ to $93 \%$ and were significantly higher than placebo.

Similar findings were reported in a randomized, placebo-controlled clinical trial conducted by Elliot and colleagues (287) in 75 HIV patients (45\% with AIDS) who had major depression. Both imipramine- and paroxetine-treated groups improved significantly compared to placebo at weeks 6,8 , and 12 . The dropout rate due to side effects was higher for those receiving imipramine (48\%) than it was for placebo $(24 \%)$ or paroxetine $(20 \%)$.

One randomized, placebo-controlled clinical trial $(\mathrm{N}=120)$ found that fluoxetine was an efficacious antidepressant agent among depressed HIV patients; the response rate for fluoxetine was $74 \%$ versus $47 \%$ for placebo (289). The same study reported significant differences between ethnic groups in terms of antidepressant efficacy; the response rates were $84 \%$ for white, $67 \%$ for Latino, and 50\% for black subjects (414). Despite the effectiveness of fluoxetine in treating depression, because both placebo response and attrition were high, the researchers suggested that the addition of another medication in patients with serious medical illness who require multiple concomitant medications may be a significant barrier to the treatment of depression.

In a double-blind, placebo-controlled trial that compared desipramine to fluoxetine in 14 women with advanced HIV disease, both agents were linked to symptom improvement; however, for most of the women significant depressive symptoms remained after the 6-week trial 
(294). These findings are consistent with those of an open-label fluoxetine trial that compared HIV-positive and HIV-negative depressed men, which found that improvement in the HIVpositive cohort was significantly delayed beyond the 8-week trial compared to the HIV-negative patients (415).

The randomized trials are supported by uncontrolled studies of depression in HIV populations and confirm the effectiveness of fluvoxamine $(295)$ and fluoxetine $(293,416,417)$ in HIV-infected asymptomatic patients and of paroxetine, sertraline, and fluoxetine in symptomatic HIV patients $(285,418)$. In an open trial in which HIV-positive depressed women were given either fluoxetine or sertraline, both drugs were found to be effective treatments (419). Another open-label study found nefazodone effective in the treatment of depressed HIV-positive patients (288). One open-label trial of 32 patients with advanced HIV disease found venlafaxine effective among depressed patients who also had minor cognitive motor disorder (420).

The use of psychostimulants for treatment of major depression in HIV-infected outpatients has been supported in smaller studies, including five case reports, one open-label trial, and one placebo-drug-placebo clinical trial $(321,322,421,422)$. These studies suggest that methylphenidate, up to $35 \mathrm{mg} / \mathrm{day}$, or dextroamphetamine, up to $10 \mathrm{mg} / \mathrm{day}$, can improve major depression.

An open trial of testosterone replacement for hypogonadal men found that of 34 study participants with major depressive disorder, 79\% had significant improvement in mood (126). There is a case report of a patient with HIV infection and comorbid major depressive disorder whose depression resolved with treatment with the antiretroviral agent zidovudine (423). For patients with severe major depression who are delusional or have failed medication treatment, ECT has been found to be safe and effective (424).

\section{b) Acute treatment of depressive disorders - psychotherapy}

Several studies of psychotherapeutic treatment of depression in HIV patients have been conducted. In a randomized, controlled trial, Targ and colleagues (425) compared structured group therapy plus fluoxetine to structured group therapy with placebo in 20 asymptomatic homosexual men with major depression or adjustment disorder and Hamilton Depression Rating Scale scores $\geq 16$. Both groups showed significant improvements in depression after 6 weeks of treatment, but there were no differences between treatment groups (425). In contrast, another randomized, placebo-controlled clinical trial that compared the efficacy and safety of fluoxetine plus group psychotherapy versus group psychotherapy alone in 47 depressed HIV-positive men for 7 weeks found that fluoxetine in addition to group therapy was more efficacious than group psychotherapy alone. Differences were particularly apparent for patients whose initial depressive episodes were rated as severe (Hamilton depression score $\geq 24$ ) (290).

Markowitz and colleagues (426) conducted a randomized, placebo-controlled clinical trial that compared 16-week interventions with interpersonal psychotherapy, cognitive behavior therapy, supportive psychotherapy, and supportive psychotherapy plus imipramine in $101 \mathrm{HIV}$ patients with depressive symptoms (Hamilton depression score $\geq 15$ ). Patients randomly assigned to interpersonal psychotherapy and supportive psychotherapy plus imipramine had significantly greater symptom improvement than those receiving supportive psychotherapy alone or cognitive behavior therapy.

In an earlier study (427), the Markowitz group demonstrated the benefits of interpersonal psychotherapy in HIV-positive, asymptomatic outpatients in an open study of men and women with DSM-III-R major depression or dysthymia. Twenty (87\%) out of 23 patients achieved a full remission after 6 weeks by clinical assessment; mean Hamilton depression scores at baseline and follow-up were 25 and 6.8, respectively. Levine et al. (428) reported that four outpatients with major depression remained euthymic for 9 months with structured group psychotherapy after acute remission was achieved with fluoxetine, $20-40 \mathrm{mg} / \mathrm{day}$. 
Other studies such as two retrospective chart reviews $(284,429)$ and a prospective effectiveness study (430) support the findings from controlled and uncontrolled treatment studies of major depression that treatment with a number of different antidepressant agents is effective and generally tolerated in HIV patients.

\section{c) Treatment of other types of depression}

Other studies have investigated the efficacy of treatment for other types of depression in HIV patients. These studies generally use screening instruments (e.g., the Center for Epidemiologic Studies Depression Scale [CES-D Scale]) rather than structured interviews or clinical evaluations to diagnose specific depressive disorders. All involved ambulatory patients who had no significant clinical manifestations of HIV disease. In a study of patients with depression (CES-D Scale score $>16$ ) but with unknown psychiatric diagnoses, investigators compared cognitive behavior therapy and brief supportive group therapy to a waiting list control condition. Outpatients $(\mathrm{N}=68)$ were randomly assigned to an 8 -week intervention of cognitive behavior therapy, support group, or a control group. While both types of psychotherapy were superior to the control condition, neither led to significant reductions on the CES-D Scale into the nondepressed range (431).

Eller (432) conducted a randomized, placebo-controlled clinical trial of alternative therapies for depression and assigned 81 male and female outpatients with HIV infection to guided imagery, relaxation therapy, or usual care. The sample had a mean CES-D Scale score of 19. There were no significant declines in CES-D Scale scores during treatment in any of the three groups.

\section{d) Treatment of mania/bipolar disorder}

One chart review (433) and one case report (434) have addressed the treatment of manic syndromes in HIV-infected patients. The chart review study identified seven male outpatients with mania who did not improve with lithium or neuroleptic treatment due to toxicity. Three patients had been treated with valproic acid (750-1750 mg/day), two with clonazepam ( $2 \mathrm{mg} /$ day), one with phenytoin, and one with carbamazepine. All seven patients experienced remission of their manic syndrome with the agent used (433). The case report described the effectiveness of valproic acid in the treatment of acute mania in a patient with AIDS complicated by dementia (434). There are no published studies of maintenance treatments for mania or bipolar disorders in HIV-infected populations.

\section{Substance use disorders}

Clinical consensus has established the necessity of treating substance use disorders in order to produce optimal psychiatric and medical outcomes for patients with HIV infection. Despite this knowledge, remarkably few studies have investigated psychiatric treatment of substance use disorders in HIV-infected patients. This section will review the literature of the treatment of substance use disorders and associated syndromes.

\section{a) Entry into a treatment program}

Entry into a treatment program is probably one of the more critical steps for the assessment and treatment of substance use disorders. Guydish and colleagues (435) described a consultation-education-triage model program that referred drug users with HIV infection to follow-up care for substance use disorders. Of the 86 patients referred over an 8 -month period, $81 \%$ were referred for further care to a substance use program, with $58 \%$ actually contacting the referral resource. Eighty percent had AIDS or symptomatic HIV disease; 37\% of patients were dependent on alcohol, $36 \%$ were dependent on amphetamines, and $26 \%$ were dependent on heroin, marijuana, or cocaine. 


\section{b) Methadone maintenance}

The Program for AIDS Counseling and Education in San Francisco is considered a model program for inner-city, poor, opiate-dependent, mostly minority men $(436,437)$. The program provides medical assessment and care, psychiatric treatment, methadone treatment, counseling, detoxification, and tolerance management for opiate addicts. Outcomes for patients enrolled in the Program for AIDS Counseling and Education have been reported in two conference papers. In one report, 29 opioid-dependent HIV patients with a mean of 28 days of drug use in the past month reduced their use to a mean of 5.9 days of use per month after 3 months in the program (438). The other report focused on clinical events related to drug use before and after enrollment for 62 patients in the program (439). Clinical events included medical complications and lapses in compliance with treatment. At baseline, patients experienced 0.72 clinically significant medical complications per 100 patient years, whereas the rate decreased significantly to 0.16 during enrollment in the Program for AIDS Counseling and Education. There was also a reduction in preventable HIV clinical events from 0.21 per 100 patient years to 0.02 per 100 patient years after starting the program. The reports provide some evidence that both the medical care as well as substance use behaviors of HIV-infected opioid addicts can benefit from a multidisciplinary, structured intervention.

\section{c) Pharmacologic treatment of substance use disorders}

One study of HIV-infected patients investigated the effect of antidepressant medications on symptoms of drug dependency, such as drug craving and comorbid depression. Batki (440) conducted a controlled trial that compared fluoxetine to placebo in 37 patients with cocaine and opiate dependency who were on a regimen of methadone maintenance. Of this sample, $41 \%$ also had major depression. Participants treated with fluoxetine ( $20-40 \mathrm{mg} /$ day) had lower median measures of cocaine metabolites in urine and fewer mean days of cocaine use per week (1.6) compared to those given placebo (2.6); the fluoxetine-treated patients also reported reduced cocaine craving. Those with major depression showed significant improvement after the 12-week treatment.

\section{d) Overall outcomes of psychiatric treatment}

Lyketsos and colleagues (430) conducted a 14-month prospective effectiveness study of clinical outcomes in HIV patients who were referred to psychiatric treatment. Within the cohort, 110 patients had current $(\mathrm{N}=66)$ or recent $(\mathrm{N}=44)$ substance use disorders; there were high rates of polysubstance dependence, with only a small proportion of patients who abused alcohol. The psychiatric treatment took place within a primary care clinic and included treatment for substance abuse and concurrent psychiatric disorders. Psychiatric intervention led to a decrease in the use of substances and better clinical condition.

\section{Anxiety disorders}

Anxiety disorders are prevalent in the population of persons who have or are at risk for HIV infection, but studies of specific treatments for anxiety disorders in HIV patients are lacking. The literature provides only one retrospective chart review and two case reports that address the management of anxiety disorders in HIV-positive patients. There are no treatment studies of PTSD among HIV-infected patients, although PTSD rates are high in some HIV-affected groups (441).

A chart review study found that buspirone was an effective anxiolytic in opioid-dependent, HIV-positive patients who were on a regimen of methadone maintenance and who also had a comorbid anxiety disorder. The study additionally showed a modest reduction of urine tests positive for substances of abuse, from $42 \%$ to $30 \%$ (328).

$\mathrm{McDaniel}$ and Johnson (442) found fluoxetine to be effective in the treatment of obsessivecompulsive disorder (OCD) in two HIV-positive patients. Both patients tolerated the fluoxetine with minimal side effects and responded with resolution of OCD symptoms within 6-8 weeks. 
A male patient with advanced AIDS complicated by comorbid trichotillomania and major depression was reported to have responded well to sertraline at a maximum dose of $150 \mathrm{mg} /$ day. The patient reported experiencing a $70 \%-80 \%$ decrease in hair pulling and a modest improvement in depressed mood, with minimal medication side effects (443).

\section{Psychotic disorders}

Nine literature citations, two of them clinical trials and the remainder case reports, present evidence concerning the efficacy of psychotropic medication for the management of psychosis in the presence of HIV infection. The nine reports contain fewer than 100 subjects, approximately $90 \%$ of them male, and most with symptomatic HIV infection, usually AIDS. Where stated, the majority did not have a history of psychosis before HIV infection, and, where stated, the majority had cognitive impairment.

Antipsychotic medications were effective in treating psychosis whether or not cognitive dysfunction or delirium was present. One large case history series found that patients with manic psychosis showed more improvement than patients with schizophreniform psychosis (315). The two clinical trials $(31,444)$ found that positive symptoms responded better than negative symptoms. Across studies the subjects, who largely had advanced HIV disease, required lower doses of antipsychotic medication, similar to the pattern seen in the elderly. Lower doses were also useful due to increased sensitivity to side effects. If a patient did not respond to or tolerate one antipsychotic, it was useful to try another from a different class $(300,301,310,312)$.

In case reports, the use of adjunctive medications, including antianxiety drugs, antidepressants, and mood stabilizers, was a common practice and often useful in individual cases, but there have been no controlled studies on the efficacy of these combinations. Two case reports, each of a single male subject with AIDS, found that catatonia responded rapidly to lorazepam $(445,446)$. No side effects were noted.

Maintenance medication was not always necessary to sustain remission of psychotic symptoms $(42,312)$. In some cases it was specifically noted that symptoms of psychosis diminished as severe late-stage medical illness progressed (42). While it has been hypothesized that there is a window of vulnerability to psychosis or mania occurring early in the course of HIV-associated dementia (447), no data support this theory, and most patients treated for psychosis have continued their maintainance medication regimens.

There are few data on the treatment of psychosis in early-stage HIV infection or among women nor is there information concerning potential modifications in the treatment of preexisting psychotic illness, which might be necessary in the presence of advancing HIV infection.

\section{Adjustment disorders}

Two open-label clinical trials have assessed the effectiveness of psychotropic medications in the management of adjustment disorders in HIV-positive patients. As part of a larger study that examined the effectiveness of paroxetine in the treatment of depression in the context of HIV infection, Grassi and colleagues (418) included five patients with a diagnosis of adjustment disorder with depressed mood. All five patients received paroxetine $(20 \mathrm{mg} /$ day $)$ and showed significant recovery as measured by the Hamilton depression scale at the 6-week endpoint.

Five male patients with neurocognitive impairment as well as a diagnosis of adjustment disorder with depressed mood were treated as part of a larger study of the effectiveness of psychostimulants in patients with HIV-associated dementia. Four of the five patients who received methylphenidate had moderate to marked improvement in symptoms; the one patient with minimal response was switched to dextroamphetamine and subsequently had a moderate response (405).

\section{Sleep disorders}

Numerous reports have documented disrupted sleep and altered sleep architecture in HIV patients $(29,448,449)$, including one study which linked fatigue and sleep disturbances to morbid- 
ity and disability in homosexual men (450). While the etiologies remain unclear, some investigators have documented growth hormone dysregulation associated with sleep pathology (451), and one study found obstructive sleep apnea due to adenotonsillar hypertrophy to be a primary cause of sleep disruption in a cohort of HIV patients with excessive daytime sleepiness (452).

The treatment of sleep disorders in HIV-positive persons has been examined in only one open-label clinical trial of flurazepam in 12 patients (453). All patients were given a one-time dose of flurazepam, $30 \mathrm{mg}$, to examine changes in the patients' electroencephalographic recordings during sleep. Flurazepam mainly affected non-REM parameters, such as reduction of times awake during the night and increases in stage two and effective sleep time. As this study did not address treatment with flurazepam beyond a one-time dose, actual treatment recommendations cannot be made.

\section{Disorders of infancy, childhood, and adolescence}

Since HIV infection in childhood is associated with developmental delays or loss of acquired skills, it is hoped that early treatment will promote normal development. Currently, it appears that antiretroviral drug therapy reduces morbidity in children, especially in those with HIVassociated neurocognitive impairment. Several studies have reported a significant increase in mental ability, as measured by the WISC-R, the McCarthy Scales of Children's Abilities, or the Bayley Scales of Infant Development, in over 450 children during 12- and 36-month trials with zidovudine $(58,246,454,455)$. None of the studies included a control group.

The safety of zidovudine for children is similar to that for adults, although up to $40 \%$ of children experience hematological side effects that require dose alterations (246). The one published study of 54 children that did not find a significant improvement in cognitive functioning with zidovudine treatment did find a significant decrease in CD4 levels over the treatment period. No data were provided on adherence to treatment or zidovudine resistance (456). Other antiretroviral agents, such as didanosine, have been studied, with a multisite project showing that combination zidovudine and didanosine therapy was superior to either used as monotherapy for most of the CNS outcomes evaluated (457). Although combination antiretroviral therapy with three agents is standard practice with adults, there is only one case report of its use with a child (458). Caregivers noted the positive impact of this regimen on an 8-year-old boy's neurocognitive development.

The effectiveness of the treatment of other mental and behavioral disorders associated with HIV infection in children and adolescents is largely unstudied. Case reports of single patients suggest that attention deficit disorders and depressive disorders in those with HIV infection may be treated by using regimens that are standard for those without HIV infection (459). As in the treatment of adults, children with HIV infection may be especially sensitive to some treatment side effects, such as insomnia or appetite suppression associated with psychostimulant use.

Pain frequently accompanies pediatric HIV and can involve multiple organ systems (460). Clinicians should be aware that the inadequate verbal skills of younger children or the fact that some children are from non-English-speaking families can lead to the underrecognition and undertreatment of pain (461).

\section{HIV-associated syndromes with psychiatric implications}

A number of HIV-associated clinical syndromes have psychiatric implications, such as wasting syndrome, fatigue, pain, and sexual dysfunction. Because there are overlapping symptoms with these conditions and psychiatric disorders, it is useful for psychiatrists to be aware of treatment studies for such syndromes so that overall clinical outcomes can be improved.

\section{a) Wasting syndrome}

Several studies have focused on the use of testosterone for treatment of wasting syndrome in both patients with low testosterone levels and patients with "clinical hypogonadism" (normal 
hormone levels). Grinspoon and colleagues (462) conducted a double-blind, placebo-controlled study of hypogonadal HIV-positive men with wasting syndrome that demonstrated the safety and efficacy of testosterone replacement therapy $(300 \mathrm{mg}$ i.m. every 3 weeks). The treatment resulted in a significant increase of lean body mass, overall quality of life, and self-perception of appearance in treated subjects. Similar findings have been reported by Rabkin and colleagues $(126,343,463)$, who conducted several randomized, placebo-controlled trials as well as open trials of testosterone replacement in men with clinical hypogonadism that led to significant positive effects on mood and weight (lean body mass). Another study that used oxymetholone, a testosterone derivative, demonstrated weight gain in cachectic men with AIDS (464). A controlled study of hypogonadal women with HIV infection that used transdermal testosterone replacement also reported improvement in lean body mass and quality of life (465).

Wagner and colleagues (466) reported an additional improvement of measures on the Brief Symptom Inventory and nutritional status for patients who exercised in addition to receiving testosterone replacement. In a double-blind, randomized, placebo-controlled trial, researchers found that resistance exercise in addition to testosterone and the anabolic steroid oxandrolone substantially increased lean body mass (467). Two open-label studies have reported that oxandrolone, $20 \mathrm{mg} /$ day, in HIV patients with wasting syndrome led to significant weight gain, in particular, increases in body cell mass $(334,335)$.

Several clinical trials have used the recombinant growth hormone somatotropin for treatment of HIV-related wasting $(336,340)$. A large multicenter, double-blind, placebo-controlled study that used growth hormone in patients with AIDS wasting reported significant improvement in overall quality of life and weight gain, with no negative effect on virological or immunological markers (339). Treatment was well tolerated.

Since tumor necrosis factor- $\alpha$ has been postulated to have a role in the pathogenesis of wasting syndrome, the effect of thalidomide, a selective inhibitor of tumor necrosis factor- $\alpha$, has been studied in patients with AIDS wasting syndrome. Two reports (a mail survey of thalidomide users and a small randomized, placebo-controlled clinical trial) have supported the positive effects of thalidomide on weight gain, appetite, and quality of life $(341,342)$.

\section{b) Fatigue}

Breitbart and colleagues (468) conducted a randomized, placebo-controlled clinical trial that used two psychostimulant medications, methylphenidate and pemoline, in treating fatigue among HIV patients. They found that both treatments were superior to placebo, with a stronger effect in the methylphenidate group. In addition, they reported that improvement in fatigue was associated with improvement in depressive symptoms and psychological distress. In an open trial of depressed patients with CD 4 counts below 200, dextroamphetamine administration led to improvement of fatigue in $95 \%$ of patients along with reduction in depression scores (322). Testosterone replacement with dehydroepiandrosterone (DHEA) in HIV-infected hypogonadal men has also been found to improve symptoms of fatigue (469).

\section{c) Pain}

Studies of the frequency and adequacy of treatment for pain among patients with AIDS find wide variations in the effectiveness of interventions to manage pain. A study by Breitbart et al. (123) found that $85 \%$ of HIV patients in their ambulatory sample treated at a cancer center received inadequate pain therapy. Yet a different study reported that only $20 \%$ of their sample of AIDS patients treated in the hospital, home care, or nursing facility did not receive effective pain control (470).

While agents such as tricyclic antidepressants and anticonvulsant medications are often used to manage chronic pain from peripheral neuropathy, published treatment studies for HIV-associated peripheral neuropathy have not supported their use. Two randomized, controlled trials have focused on HIV-associated peripheral neuropathy; both trials used the agent mexiletine, 
an antiarrhythmic drug with local anesthetic properties that had been used in the treatment of diabetic neuropathy $(317,471)$. Neither study found pain relief different from that of placebo. Furthermore, Kieburtz et al. (317) did not find that treatment with amitriptyline provided significant pain relief compared to placebo. Shlay and colleagues (472) conducted a randomized, controlled trial that compared acupuncture, amitriptyline, and placebo for pain due to HIVrelated peripheral neuropathy and found that neither acupuncture nor amitriptyline was more effective than placebo.

\section{d) Sexual dysfunction}

Sexual dysfunction is experienced commonly by HIV patients, both men and women, particularly with HIV disease progression. While no treatment trials are currently published, a cautionary note regarding sildenafil is clinically important. Sildenafil should be used judiciously in patients with erectile dysfunction who are also taking protease inhibitors, since the primary route of sildenafil metabolism is via the cytochrome P450 isoenzyme 3A4. One death has been reported in a 47-year-old male taking ritonavir and saquinavir who was administered $25 \mathrm{mg}$ of sildenafil (473). The manufacturer of sildenafil has recommended that patients receiving ritonavir should not take more than a single sildenafil dose of $25 \mathrm{mg}$ in a 48 -hour period.

\section{PART C: \\ FUTURE RESEARCH NEEDS}

Considerable progress has been made in understanding HIV, AIDS, and treatment for these conditions. However, further studies are needed regarding specific, effective treatments for patients, including women and children, with HIV/AIDS. Areas of specific concern include:

1. The impact of antiretroviral therapy on the incidence of CNS opportunistic diseases and neuropsychiatric disorders.

2. The specific etiology of HIV-associated dementia and minor cognitive motor disorder and effective interventions to prevent or ameliorate these conditions.

3. The clinical significance of drug-drug interactions of psychotropic agents and HIVrelated medications.

4. Whether HIV is directly linked to the occurrence of psychosis, mania, depression, or other psychiatric disorders in persons with HIV infection.

5. Whether the CNS serves as a reservoir of HIV-resistant strains and the clinical implications if it does.

6. The effectiveness of psychiatric care in critical areas of treatment adherence, prevention of HIV infection, and psychosocial adaptation.

7. Information about the impact of HIV infection on development in children, with possible differences in clinical course due to whether infected in utero or perinatally or due to type of treatment received.

8. More effective interventions for HIV risk reduction in specific psychiatric populations, particularly for primary prevention of HIV infection.

9. The need for more data about the effectiveness of psychiatric treatments such as psychotherapy, pharmacotherapy, and psychosocial services for adults, adolescents, and children with HIV/AIDS.

10. Investigating possible gender differences in the problems associated with HIV/AIDS and treatment for these conditions. 
APPENDIX 1. HIV/AIDS Electronic Resources

\begin{tabular}{|c|c|c|}
\hline Resource & Organization & URL \\
\hline General information & $\begin{array}{l}\text { AIDS Action Committee (AAC) } \\
\text { AIDS Daily Summary } \\
\text { AIDS Education Training Centers (AETC) } \\
\text { AIDS Virtual Library } \\
\text { American Medical Association } \\
\text { American Psychiatric Association } \\
\text { Business and Labor Resource Service } \\
\text { Centers for Disease Control and Prevention (CDC) } \\
\text { CDC National Prevention Information Network (CDCNAC) } \\
\text { CDC National AIDS Funding Database } \\
\text { CMV Retinitis Education and Treatment Information } \\
\text { Food and Drug Administration (FDA) } \\
\text { FDA/OASHI: HIV/AIDS } \\
\text { FDA Medical Bulletin } \\
\text { Healthcare Communications Group } \\
\text { Healthfinder } \\
\text { HIVDent } \\
\text { Human Retroviruses and AIDS Sequence Database } \\
\text { Johns Hopkins AIDS Service } \\
\text { Infectious Diseases Society } \\
\text { Insights in HIV Disease Management } \\
\text { Medical Matrix } \\
\text { National Academy of Sciences } \\
\text { National AIDS Treatment Advocacy Project (NATAP) } \\
\text { National Hemophilia Foundation (NHF) } \\
\text { National Institutes of Health (NIH) } \\
\text { National Science Foundation } \\
\text { New York Online Access on Health } \\
\text { Pharmaceutical Research Manufacturers Association } \\
\text { of America (PhRMA) }\end{array}$ & $\begin{array}{l}\text { www.aac.org } \\
\text { www.cdcnpin.org/news/start.htm } \\
\text { www.service.emory.edu/SEATEC/AETCdir.html\#USA } \\
\text { www.planetq.com/aidsvl/index.html } \\
\text { www.ama-assn.org } \\
\text { www.psych.org/aids } \\
\text { www.brta-hta.org } \\
\text { www.cdc.gov } \\
\text { www.cdcpin.org } \\
\text { www.cdcnpin.org/db/public/fundmain.htm } \\
\text { www.piv.org } \\
\text { www.fda.gov } \\
\text { www.fda.gov/oashi/aids/hiv.gov } \\
\text { www.fda.gov/medbull/contents.html } \\
\text { www.healthcg.com } \\
\text { www.healthfinder.gov } \\
\text { www.hivdent.org } \\
\text { hiv-web.ianl.gov } \\
\text { www.hopkins-aids.edu } \\
\text { www.idsociety.org } \\
\text { www.meniscius.com/hiv } \\
\text { www.medmatrix.org/index.asp } \\
\text { www.nas.edu } \\
\text { www.natap.org } \\
\text { www.infoahf.org } \\
\text { www.nih.gov } \\
\text { www.nsf.gov } \\
\text { www.noah.cuny.edu/aids/aids.html } \\
\text { www.phrma.org }\end{array}$ \\
\hline
\end{tabular}


APPENDIX 1. HIV/AIDS Electronic Resources (continued)

\begin{tabular}{|c|c|c|}
\hline Resource & Organization & URL \\
\hline $\begin{array}{l}\text { General information } \\
\text { (continued) }\end{array}$ & $\begin{array}{l}\text { The Access Project } \\
\text { The Body } \\
\text { University of California HIV/AIDS Program } \\
\text { USP Pharmacopeia Practitioners Reporting Network } \\
\text { Yahoo: Health Medicines HIV/AIDS } \\
\text { Webber's AIDS/HIV Law and Policy Resource }\end{array}$ & $\begin{array}{l}\text { 204.179.124.69/network/access/index.html } \\
\text { www.thebody.com } \\
\text { hivinsite.ucsf.edu } \\
\text { usp.org/index } \\
\text { www.yahoo.com/Health/Medicine } \\
\text { www.critpath.org/aidslaw }\end{array}$ \\
\hline $\begin{array}{l}\text { Alternative } \\
\text { treatments }\end{array}$ & $\begin{array}{l}\text { American Chiropractic Association } \\
\text { ATDN's Alternative Treatments } \\
\text { Bastyr University AIDS Research Center } \\
\text { Herb Research Foundation } \\
\text { Homeopathic Educational Services } \\
\text { Institute for Traditional Medicine }\end{array}$ & $\begin{array}{l}\text { www.amerchiro.org/aca } \\
\text { 204.179.124.69/network/altx.html } \\
\text { www.bastyr.edu/research/busrc } \\
\text { www.herbs.org } \\
\text { www.homeopathic.com } \\
\text { www.europa.com/-itm }\end{array}$ \\
\hline Clinical trials & $\begin{array}{l}\text { Adult AIDS Clinical Trials Group (ACTG) } \\
\text { AIDS Clinical Trial Information Services } \\
\text { AIDS Treatment Information Service } \\
\text { American Foundation for AIDS Research (amFAR) } \\
\text { Canadian HIV Trials Network } \\
\text { Pediatric AIDS Clinical Trials Group } \\
\text { The Body } \\
\text { USA HIV Clinical Trials/Trial Search } \\
\text { Vanderbilt Vaccines }\end{array}$ & $\begin{array}{l}\text { aactg.a-3.com/links.com } \\
\text { www.actic.org } \\
\text { www.atis.org } \\
\text { www.amfar.org/td } \\
\text { www.hivnet.ubc.ca/ctn.html } \\
\text { pactg.s-3.com/links.htm } \\
\text { www.thebody.com/treatclintri.html } \\
\text { hivinsite.ucsf.edu/tsearch } \\
\text { www.mc.vanderbilt.edu/adl/aids_project/vac }\end{array}$ \\
\hline $\begin{array}{l}\text { Harm reduction/ } \\
\text { needle exchange }\end{array}$ & $\begin{array}{l}\text { Center for AIDS Prevention Studies (UCSF) } \\
\text { The Lindesmith Center } \\
\text { The XCHANGE POINT } \\
\text { North American Syringe Exchange Network } \\
\text { Safe Works AIDS Project }\end{array}$ & $\begin{array}{l}\text { www.caps.ucsf.edu/capsweb } \\
\text { www.lindesmith.org } \\
\text { members.aol.com/xchangept/aids.html } \\
\text { www.nasen.org } \\
\text { www.safeworks.org }\end{array}$ \\
\hline
\end{tabular}


APPENDIX 1. HIV/AIDS Electronic Resources (continued)

\begin{tabular}{lll}
\hline Resource & Organization & URL \\
\hline $\begin{array}{l}\text { Recreational drugs and } \\
\text { anti-HIV drug }\end{array}$ & National AIDS Manual & www.nam.org/uk/atu/atu51.trt \\
information & Notes from the Underground & www.aidsinfonyc.org/pwahg/notes/38.html \\
& Positively AWARE & ww.tpan.com/library/pa/mayjun97/p.32.htm \\
& The Toronto Hospital & ww.tthhivclinic.com/recreation.htm \\
& POZ & www.thebody.com/poz/survival/6_98/warning.html \\
& CATIE (Community AIDS Treatment Information Exchange) & www.catie.ca \\
& University of California, San Francisco & Hivinsite.ucsf.edu/topics/substance_use/ \\
hiv_aids_medications_and_interventions_with_legal_ & and_illegal_drugs/ \\
& & www.medscape.com/medscape/hiv/clinicalmgmt/can/drug/ \\
& public/toccm.drug.html \\
& Medscape & www.crescentcity.com/noaids \\
& wo/AIDS Task Force & www.afa.org/treatment/beta/b34/b34piint.html \\
& Project Inform & \\
San Francisco AIDS Foundation &
\end{tabular}




\section{INDIVIDUALS AND ORGANIZATIONS THAT SUBMITTED COMMENTS}

J. Atkinson, M.D.

Steven Batki, M.D.

Christy L. Beaudin, Ph.D., L.C.S.W., C.P.H.Q.

Carl Bell, M.D.

Marcia Bennett, D.N.S.

Alan Berkman, M.D.

Philip A. Bialer, M.D.

Charles H. Blackinton, M.D., P.A.

Mel Blaustein, M.D.

Michael T. Brady, M.D.

William Breitbart, M.D.

Robert Broadbent, M.D.

Robert Paul Cabaj, M.D.

Susan Chuck, Pharm.D.

Norman A. Clemens, M.D.

Dave Davis, M.D.

Steven A. Epstein, M.D.

Abraham Feingold, Psy.D.

Steve Ferrando, M.D.

Jack Gorman, M.D.

William M. Greenberg, M.D.

Susan Haikalis, L.C.S.W.

Mark Halman, M.D.

Lawrence Hartmann, M.D.

Roman M. Hendrickson, M.D.

Al Herzog, M.D.

Dan Hicks, M.D.

Ewald Horwath, M.D.
Michael Hughes, M.D.

Jeffrey S. Janofsky, M.D.

T. Stephen Jones, M.D.

Nalini V. Juthani, M.D.

Cheryl Ann Kennedy, M.D.

Robert Kertzner, M.D.

Lawrence Y. Kline, M.D.

Joyce Kobayashi, M.D.

Ronald R. Koegler, M.D.

Thomas Kosten, M.D.

Zev Labins, M.D.

Debra E. Lyon, Ph.D.

John C. Markowitz, M.D.

Elinore F. McCance-Katz, M.D.

Karen McKinnon, M.A.

Philip R. Muskin, M.D.

Judith Rabkin, M.D.

David Rosmarin, M.D.

Peter Ross

Pedro Ruiz, M.D.

Larry Siegel, M.D.

Robert Stasko, M.D.

William R. Tatomer, M.D.

Joshua T. Thornhill IV, M.D.

Glenn J. Treisman, M.D.

Milton L. Wainberg, M.D.

Bert Warren, M.D.

Jonathan L. Worth, M.D.

Claire Zilber, M.D.

\author{
American Academy of Family Physicians \\ American Academy of Psychiatry and the Law \\ American Group Psychotherapy Association, Inc. \\ American Psychiatric Nurses Association \\ American Society of Addiction Medicine \\ American Society of Clinical Psychopharmacology, Inc. \\ APA Commission on Psychotherapy by Psychiatrists \\ Association for Academic Psychiatry \\ Association of Gay and Lesbian Psychiatrists \\ Black Psychiatrists of America \\ National Association of Social Workers \\ PacifiCare Behavioral Health \\ Royal Australian and New Zealand College of Psychiatrists \\ San Francisco AIDS Foundation
}


The following coding system is used to indicate the nature of the supporting evidence in the references:

[A] Randomized clinical trial. A study of an intervention in which subjects are prospectively followed over time; there are treatment and control groups; subjects are randomly assigned to the two groups; both the subjects and the investigators are blind to the assignments.

[B] Clinical trial. A prospective study in which an intervention is made and the results of that intervention are tracked longitudinally; study does not meet standards for a randomized clinical trial.

[C] Cohort or longitudinal study. A study in which subjects are prospectively followed over time without any specific intervention.

[D] Case-control study. A study in which a group of patients and a group of control subjects are identified in the present and information about them is pursued retrospectively or backward in time.

[E] Review with secondary data analysis. A structured analytic review of existing data (e.g., a meta-analysis or a decision analysis).

[F] Review. A qualitative review and discussion of previously published literature without a quantitative synthesis of the data.

[G] Other. Textbooks, expert opinion, case reports, and other reports not included above.

1. Ho DD: Viral counts count in HIV infection. Science 1996; 272:1124-1125 [G]

2. National Center for Health Statistics: AIDS falls from top ten causes of death; teen births, infant mortality, homicide all decline (press release). Hyattsville, Md, US Department of Health and Human Services, Oct 7, 1998 [G]

3. Centers for Disease Control and Prevention: HIV/AIDS Surveillance Report 1999; 1(11) [G]

4. Steinbrook R: Caring for people with human immunodeficiency virus infection (editorial). N Engl J Med 1998; 339:1926-1928 [G]

5. Mitka M: Slowing decline in AIDS deaths prompts concern. JAMA 1999; 282:1216-1217 [G]

6. Counselling Guidelines for HIV Testing. Ottawa, Canadian Medical Association, 1995 [G]

7. Gostin LO, Lazzarini Z, Jones TS, Flaherty K: Prevention of HIV/AIDS and other bloodborne diseases among injection drug users: a national survey on the regulation of syringes and needles. JAMA 1997; 277:53-62 [D]

8. Kinloch-de Loes S, de Saussure P, Saurat JH, Stalder H, Hirschel B, Perrin LH: Symptomatic primary infection due to human immunodeficiency virus type 1: review of 31 cases. Clin Infect Dis 1993; 17:59-65 [D]

9. Zhang Z, Schuler T, Zupancic M, Wietgrefe S, Staskus KA, Reimann KA, Reinhart TA, Rogan M, Cavert W, Miller CJ, Veazey RS, Notermans D, Little S, Danner SA, Richman DD, Havlir D, Wong J, Jordan HL, Schacker TW, Racz P, Tenner-Racz K, Letvin NL, Wolinsky S, Haase AT: Sexual transmission and propagation of SIV and HIV in resting and activated CD4+ T cells. Science 1999; 286:1353-1357 [G]

10. Chun TW, Engel D, Berrey MM, Shea T, Corey L, Fauci AS: Early establishment of a pool of latently infected, resting CD4(+) T cells during primary HIV-1 infection. Proc Natl Acad Sci USA 1998; 95:8869-8873 [G]

11. Schrager LK, D'Souza MP: Cellular and anatomical reservoirs of HIV-1 in patients receiving potent antiretroviral combination therapy. JAMA 1998; 280:67-71 [F] 
12. Hengge UR, Brockmeyer NH, Esser S, Maschke M, Goos M: HIV-1 RNA levels in cerebrospinal fluid and plasma correlate with AIDS dementia (letter). AIDS 1998; 12:818$820[\mathrm{G}]$

13. Hirsch MS, Conway B, D’Aquila RT, Johnson VA, Brun-Vezinet F, Clotet B, Demeter LM, Hammer SM, Jacobsen DM, Kuritzkes DR, Loveday C, Mellors JW, Vella S, Richman DD (International AIDS Society-USA Panel): Antiretroviral drug resistance testing in adults with HIV infection: implications for clinical management. JAMA 1998; 279:1984-1991 [E]

14. Hughes MD, Johnson VA, Hirsch MS, Bremer JW, Elbeik T, Erice A, Kuritzkes DR, Scott WA, Spector SA, Basgoz N, Fischl MA, D’Aquila RT (ACTG 241 Protocol Virology Substudy Team): Monitoring plasma HIV-1 RNA levels in addition to CD4+ lymphocyte count improves assessment of antiretroviral therapeutic response. Ann Intern Med 1997; 126:929-938 [A]

15. O'Brien WA, Hartigan PM, Daar ES, Simberkoff MS, Hamilton JD (VA Cooperative Study Group on AIDS): Changes in plasma HIV RNA levels and CD4+ lymphocyte counts predict both response to antiretroviral therapy and therapeutic failure. Ann Intern Med 1997; 126:939-945 [A]

16. Paul WE (ed): Fundamental Immunology, 3rd ed. Philadelphia, Lippincott-Raven, 1994, p 1386 [G]

17. Centers for Disease Control and Prevention: 1993 revised classification system for HIV infection and expanded surveillance case definition for AIDS among adolescents and adults. MMWR Morb Mortal Wkly Rep 1992; 41(RR-17):1-19 [E]

18. Descamps D, Flandre P, Calvez V, Peytavin G, Meiffredy V, Collin G, Delaugerre C, RobertDelmas S, Bazin B, Aboulker JP, Pialoux G, Raffi F, Brun-Vezinet F: Mechanisms of virologic failure in previously untreated HIV-infected patients from a trial of inductionmaintenance therapy. JAMA 2000; 283:205-211 [G]

19. Deeks SG, Smith M, Holodniy M, Kahn JO: HIV-1 protease inhibitors: a review for clinicians. JAMA 1997; 277:145-153 [F]

20. Boden D, Hurley A, Zhang L, Cao Y, Guo Y, Jones E, Tsay J, Ip J, Farthing C, Limoli K, Parkin N, Markowitz M: HIV-1 drug resistance in newly infected individuals. JAMA 1999; 282:1135-1141 [D]

21. Pomerantz RJ: Primary HIV-1 resistance: a new phase in the epidemic? (editorial). JAMA 1999; 282:1177-1179 [G]

22. Swindells S, Zheng J, Gendelman HE: HIV-associated dementia: new insights into disease pathogenesis and therapeutic interventions. AIDS Patient Care STDS 1999; 3:153-163 [F]

23. Zink WE, Zheng J, Persidsky Y, Poluektova L, Gendelman HE: The neuropathogenesis of HIV-1 infection. FEMS Immunol Med Microbiol 1999; 26:233-241 [F]

24. Kolson DL, Lavi E, Gonzalez-Scarano F: The effects of human immunodeficiency virus in the central nervous system. Adv Virus Res 1998; 50:1-47 [F]

25. Childs EA, Lyles RH, Selnes OA, Chen B, Miller EN, Cohen BA, Becker JT, Mellors J, McArthur JC: Plasma viral load and CD4 lymphocytes predict HIV-associated dementia and sensory neuropathy. Neurology 1999; 52:607-613 [C]

26. Inkina N, Price RW, Barbour J, Bandrapalli N, Novakovic-Agopian T, Staprans S, Grant RM: HIV-1 compartmentalization and discordant virologic failure in CSF and plasma (abstract 297). Sixth Conference on Retroviruses and Opportunistic Infections 1999; p 124 $[\mathrm{G}]$

27. Haas DW, Spearman P, Johnson B, Harris VL, Donlon R, Wilkinson GR, Clough LA, Grosso RA, Stevens MR: Discordant HIV-1 RNA decay in CSF versus plasma following initiation of antiretroviral therapy: a prospective ultra-intensive CSF sampling study (abstract 405), in Abstracts From the 6th Conference on Retroviruses and Opportunistic Infections. Alexandria, VA, Foundation for Retrovirology and Human Health, 1999 [C] 
28. McDaniel JS, Campos PE, Purcell DW, Farber EW, Bondurant A, Donahoe JE, Chang BM: A national, randomized survey of HIV/AIDS attitudes and knowledge among psychiatrists-in-training. Academic Psychiatry 1998; 22:107-116 [G]

29. White JL, Darko DF, Brown SJ, Miller JC, Hayduk R, Kelly T, Mitler MM: Early central nervous system response to HIV infection: sleep distortion and cognitive-motor decrements. AIDS 1995; 9:1043-1050 [C]

30. Wilkie FL, Eisdorfer C, Morgan R, Loewenstein DA, Szapocznik J: Cognition in early human immunodeficiency virus infection. Arch Neurol 1990; 47:433-440 [G]

31. Sewell DD, Jeste DV, McAdams LA, Bailey A, Harris MJ, Atkinson JH, Chandler JL, McCutchan JA, Grant I (HNRC Group): Neuroleptic treatment of HIV-associated psychosis. Neuropsychopharmacology 1994; 10:223-229 [B]

32. Mijch AM, Judd FK, Lyketsos CG, Ellen S, Cockram A: Secondary mania in patients with HIV infection: are antiretrovirals protective? J Neuropsychiatry Clin Neurosci 1999; 11: 475-480 [G]

33. Ellen SR, Judd FK, Mijch AM, Cockram A: Secondary mania in patients with HIV infection. Aust NZ J Psychiatry 1999; 33:353-360 [G]

34. Lyketsos CG, Schwartz J, Fishman M, Treisman G: AIDS mania. J Neuropsychiatry Clin Neurosci 1997; 9:277-279 [D]

35. Nomenclature and research case definitions for neurologic manifestations of human immunodeficiency virus-type 1 (HIV-1) infection: report of a working group of the American Academy of Neurology AIDS Task Force. Neurology 1991; 41:778-785 [F]

36. Masliah E, Ge N, Mucke L: Pathogenesis of HIV-1 associated neurodegeneration. Crit Rev Neurobiol 1996; 10:57-67 [F]

37. Mayeux R, Stern Y, Tang M-X, Todak G, Marder K, Sano M, Richards M, Stein Z, Ehrhardt AA, Gorman JM: Mortality risks in gay men with human immunodeficiency virus infection and cognitive impairment. Neurology 1993; 43:176-182 [C]

38. Ellis RJ, Deutsch R, Heaton RK, Marcotte TD, McCutchan JA, Nelson JA, Abramson I, Thal LJ, Atkinson JH, Wallace MR, Grant I (San Diego HIV Neurobehavioral Research Center Group): Neurocognitive impairment is an independent risk factor for death in HIV infection. Arch Neurol 1997; 54:416-424 [G]

39. Simpson DM: Human immunodeficiency virus-associated dementia: review pf pathogenesis, prophylaxis, and treatment studies of zidovudine therapy. Clin Infect Dis 1999; 29: 19-34 [F]

40. Sacktor NC, Lyles RH, Skolasky RL, Anderson DE, McArthur JC, McFarlane G, Selnes OA, Becker JT, Cohen B, Wesch J, Miller EN: Combination antiretroviral therapy improves psychomotor speed performance in HIV-seropositive homosexual men: Multicenter AIDS Cohort Study (MACS). Neurology 1999; 52:1640-1647 [B]

41. Dore GJ, Correll PK, Li Y, Kaldor JM, Cooper DA, Brew BJ: Changes to AIDS dementia complex in the era of highly active antiretroviral therapy. AIDS 1999; 13:1249-1253 [G]

42. Halstead S, Riccio M, Harlow P, Oretti R, Thompson C: Psychosis associated with HIV infection. Br J Psychiatry 1988; 153:618-623 [G]

43. Harris M, Jeste D, Gleghorn A, Sewell D: New-onset psychosis in HIV-infected patients. J Clin Psychiatry 1991; 52:369-376 [E]

44. Goodkin K: Psychiatric disorders in HIV-spectrum illness. Tex Med 1988; 84(9):55-61 [F]

45. Maj M: Organic mental disorders in HIV-1 infection. AIDS 1990; 4:831-840 [G]

46. Breitbart W, Marotta R, Platt M, Weisman H, Derevenco M, Grau C, Corbera K, Raymond S, Lund S, Jacobson P: A double-blind trial of haloperidol, chlorpromazine, and lorazepam in the treatment of delirium in hospitalized AIDS patients. Am J Psychiatry 1996; 153: 231-237 [A]

47. American Psychiatric Association: Practice Guideline for the Treatment of Patients With Delirium. Am J Psychiatry 1999; 156(May suppl):1-20 [G] 
48. McKegney FP, Aronson MK, Oot WL: Identifying depression in the old old. Psychodynamics 1988; 29:175-181 [G]

49. Product monograph: Sustiva ${ }^{\mathrm{TM}}$ (efavirenz). Wilmington, DE, Dupont Pharmaceuticals, $1999[\mathrm{~F}]$

50. Bialer PA, Wallack JJ, McDaniel JS: Human immunodeficiency virus and AIDS, in Psychiatric Care of the Medical Patient. Edited by Stoudemire A, Fogel BS, Greenberg DB. New York, Oxford University Press, 2000, pp 871-888 [G]

51. McDaniel JS, Purcell DW, Farber EW: Severe mental illness and HIV-related medical and neuropsychiatric sequelae. Clin Psychol Rev 1997; 17:311-325 [G]

52. Grant I, Atkinson JH Jr: Neuropsychiatric aspects of HIV infection and AIDS, in Kaplan and Sadock's Comprehensive Textbook of Psychiatry. Edited by Sadock BJ, Sadock VA. Philadelphia, Lippincott Williams \& Wilkins, 1999, pp 308-336 [G]

53. European Collaborative Study: Natural history of vertically acquired human immunodeficiency virus-1 infection. Pediatrics 1994; 94:815-819 [F]

54. Pavlakis SG, Frank Y, Nocyze M, Porricolo M, Prohovnik I, Wiznia A: Acquired immunodeficiency syndrome and the developing nervous system. Adv Pediatr 1994; 41:427-451 $[\mathrm{F}]$

55. Mintz M: Neurological and developmental problems in pediatric HIV infection. J Nutr 1996; 126 (suppl 10):2663S-2673S [F]

56. Cooper ER, Hanson C, Diaz C, Mendez H, Abboud R, Nugent R, Pitt J, Rich K, Rodriguez EM, Smeriglio V (Women and Infants Transmission Study Group): Encephalopathy and progression of human immunodeficiency virus disease in a cohort of children with perinatally acquired human immunodeficiency virus infection. J Pediatr 1998; 132:808-812 [C]

57. Bachanas PJ, Kullgren KA, Morris MK, Jones JS: Influence of family factors and illness parameters on HIV-infected children's cognitive, academic and psychological functioning. NIMH Conference on the Role of Families in Adapting to and Preventing HIV/AIDS. Washington, DC, July, 1998 [D]

58. Wolters P, Brouwers P, Moss H, Pizzo P: Adaptive behavior of children with symptomatic HIV infection before and after zidovudine therapy. J Pediatr Psychol 1994; 19:47-61 [B]

59. Frank EG, Foley GM, Kuchuk A: Cognitive functioning in school-age children with human immunodeficiency virus. Percept Mot Skills 1997; 85:267-272 [G]

60. Atkinson JH Jr, Grant I, Kennedy CJ, Richman DD, Spector SA, McCutchan JA: Prevalence of psychiatric disorders among men infected with human immunodeficiency virus: a controlled study. Arch Gen Psychiatry 1988; 45:859-864 [D]

61. Brown GR, Rundell JR, McManis SE, Kendall SN, Zachary R, Temoshok L: Prevalence of psychiatric disorders in early stages of HIV infection. Psychosom Med 1992; 54:588-601 [D]

62. Williams JB, Rabkin JG, Remien RH, Gorman JM, Ehrhardt AA: Multidisciplinary baseline assessment of homosexual men with and without human immunodeficiency virus infection, II: standardized clinical assessment of current and lifetime psychopathology. Arch Gen Psychiatry 1991; 48:124-130 [D]

63. McDaniel JS, Fowlie E, Summerville MB, Farber EW, Cohen-Cole SA: An assessment of rates of psychiatric morbidity and functioning in HIV disease. Gen Hosp Psychiatry 1995; 17:346-352 [D]

64. Uldall KK, Koutsky LA, Bradshaw DH, Krone M: Use of hospital services by AIDS patients with psychiatric illness. Gen Hosp Psychiatry 1998; 20:292-301 [D]

65. Mayne TJ, Vittinghoff E, Chesney MA, Barrett DC, Coates TJ: Depressive affect and survival among gay and bisexual men infected with HIV. Arch Intern Med 1996; 156:2233$2238[\mathrm{D}]$

66. Lyketsos CG, Hutton H, Fishman M, Schwartz J, Treisman GJ: Psychiatric morbidity on entry to an HIV primary care clinic. AIDS 1996; 10:1033-1039 [G] 
67. Acuff C, Archambeault J, Greenberg B: Mental Health Care for People Living With or Affected by HIV/AIDS: A Practical Guide (SAMHSA Monograph).

www.mentalhealth.org/cmhs/HIVAIDS/mhcarehiv.htm [G]

68. Rabkin JG, Johnson J, Lin SH, Lipsitz JD, Remien RH, Williams JB, Gorman JM: Psychopathology in male and female HIV-positive and negative injecting drug users: longitudinal course over 3 years. AIDS 1997; 11:507-515 [G]

69. Rabkin JG, Ferrando SJ, Jacobsberg LB, Fishman B: Prevalence of axis I disorders in an AIDS cohort: a cross-sectional, controlled study. Compr Psychiatry 1997; 38:146-154 [D]

70. Rosenberger PH, Bornstein RA, Nasrallah HA, Para MF, Whitaker CC, Fass RJ, Rice RR Jr: Psychopathology in human immunodeficiency virus infection: lifetime and current assessment. Compr Psychiatry 1993; 34:150-158 [D]

71. Perry S, Jacobsberg LB, Fishman B, Frances A, Bobo J, Jacobsberg BK: Psychiatric diagnosis before serological testing for the human immunodeficiency virus. Am J Psychiatry 1990; 147:89-93 [G]

72. Perkins DO, Stern RA, Golden RN, Murphy C, Naftolowitz D, Evans DL: Mood disorders in HIV infection: prevalence and risk factors in a nonepicenter of the AIDS epidemic. Am J Psychiatry 1994; 151:233-236 [D]

73. Lipsitz JD, Williams JB, Rabkin JG, Remien RH, Bradbury M, el Sadr W, Goetz R, Sorrell S, Gorman JM: Psychopathology in male and female intravenous drug users with and without HIV infection. Am J Psychiatry 1994; 151:1662-1668 [D]

74. McKinnon K, Cournos F, Sugden R, Guido JR, Herman R: The relative contributions of psychiatric symptoms and AIDS knowledge to HIV risk behaviors among people with severe mental illness. J Clin Psychiatry 1996; 57:506-513 [D]

75. Zierler S, Cunningham WE, Andersen R, Shapiro MF, Nakazono T, Morton S, Crystal S, Stein M, Turner B, St Clair P, Bozette SA: Violence victimization after HIV infection in a US probability sample of adult patients in primary care. Am J Public Health 2000; 90:208$215[\mathrm{G}]$

76. Kassler WJ, Wu AW: Addressing HIV infection in office practice: assessing risk, counseling, and testing. Prim Care 1992; 19:19-33 [F]

77. APA Commission on AIDS: Guidelines for HIV Antibody Testing. Washington, DC, APA, $1998[\mathrm{G}]$

78. American College of Obstetricians and Gynecologists: OB-GYNS Revise Screening Recommendations; Expand Testing for Diabetes, HIV and Hepatitis C Advised (press release). Washington, DC, ACOG, Nov 30, 1999 [G]

79. Purcell DW, DeGroff AS, Wolitski RJ: HIV prevention case management: current practice and future directions. Health Soc Work 1998; 23:282-289 [E]

80. Division of HIV/AIDS Prevention: Condoms and Their Use in Preventing HIV Infection and Other STDs. Atlanta, CDC National Center for HIV, STD and TB Prevention, 1999 [G]

81. Katz MH, Gerberding JL: Postexposure treatment of people exposed to the human immunodeficiency virus through sexual contact or injection-drug use. N Engl J Med 1997; 336:1097-1100 [G]

82. Centers for Disease Control and Prevention: Public health service guidelines for the management of health-care worker exposures to HIV and recommendations for postexposure prophylaxis. MMWR Morb Mortal Wkly Rep 1998; 47:1-39 [G]

83. Riley D, Sawka E, Conley P, Hewitt D, Mitic W, Poulin C, Room R, Single E, Topp J: Harm reduction: concepts and practice: a policy discussion paper. Subst Use Misuse 1999; 34:9-24 [F]

84. Ferrando SJ: Substance abuse and HIV. Psychiatric Annals (in press) [F]

85. APA Commission on AIDS: Position statement on needle exchange programs. Washington, DC, APA, $1996[\mathrm{G}]$ 
86. Des Jarlais D, Friedman P, Hagan H, Friedman SR: The protective effect of AIDS-related behavioral change among injection drug users: a cross-national study. Am J Public Health 1996; 86:1780-1785 [C]

87. Cournos F, McKinnon K: HIV seroprevalence among people with severe mental illness in the United States: a critical review. Clin Psychol Rev 1997; 17:259-269 [F]

88. Cournos F, Guido JR, Coomaraswamy S, Meyer-Bahlburg H, Sugden R, Horwath E: Sexual activity and risk of HIV infection among patients with schizophrenia. Am J Psychiatry 1994; 151:228-232 [G]

89. Sullivan G, Koegel P, Kanouse DE, Cournos F, McKinnon K, Young AS, Bean D: HIV and people with serious mental illness: the public sector's role in reducing HIV risk and improving care. Psychiatr Serv 1999; 50:648-652 [F]

90. Brown EJ, Jemmott LS: HIV among people with mental illness: contributing factors, prevention needs, barriers, and strategies. J Psychosoc Nurs Ment Health Serv 2000; 38:14-19 [G]

91. Weinhardt LS, Carey MP, Carey KB, Verdecias RN: Increasing assertiveness skills to reduce HIV risk among women living with a severe and persistent mental illness. J Consult Clin Psychol 1998; 66:680-684 [A]

92. Kelly JA: HIV risk reduction interventions for persons with severe mental illness. Clin Psychol Rev 1997; 17:293-309 [F]

93. Bamberger JD, Waldo CR, Gerberding JL, Katz MH: Postexposure prophylaxis for human immunodeficiency virus (HIV) infection following sexual assault. Am J Med 1999; 106: 323-326 [F]

94. APA Commission on AIDS: Position statement on confidentiality, disclosure, and protection of others. Am J Psychiatry 1993; 150:852 [G]

95. Paterson DL, Swindells S, Mohr J, Brester M, Vergis EN, Squier C, Wagener MM, Singh $\mathrm{N}$ : Adherence to protease inhibitor therapy and outcomes in patients with HIV infection. Ann Intern Med 2000; 133:21-30 [C]

96. Singh N, Squier C, Sivek C, Wagener M, Nguyen MH, Yu VL: Determinants of compliance with antiretroviral therapy in patients with human immunodeficiency virus: prospective assessment with implications for enhancing compliance. AIDS Care 1996; 8:261-269 [C]

97. Perry S, Jacobsberg L, Card CA, Ashman T, Frances A, Fishman B: Severity of psychiatric symptoms after HIV testing. Am J Psychiatry 1993;150:775-779 [C]

98. Somlai AM, Heckman TG, Kelly JA, Mulry GW, Multhauf KE: The response of religious congregations to the spiritual needs of people living with HIV/AIDS. J Pastoral Care 1997; 51:415-426[G]

99. Stoll R: Guidelines for spiritual assessment. Am J Nursing 1979; 79:1574-1577 [G]

100. Mouton C, Teno JM, Mor V, Piette J: Communication of preferences for care among human immunodeficiency virus-infected patients: barriers to informed decisions? Arch Fam Med 1997; 6:342-347 [G]

101. Folstein MF, Folstein SE, McHugh PR: "Mini-Mental State": a practical method for grading the cognitive state of patients for the clinician. J Psychiatr Res 1975; 12:189-198 [G]

102. Jones BN, Teng EL, Folstein MF, Harrison KS: A new bedside test of cognition for patients with HIV infection. Ann Intern Med 1993; 119:1001-1004 [C]

103. Power C, Selnes OA, Grim JA, McArthur JC: HIV Dementia Scale: a rapid screening test. Acquir Immune Defic Syndr Hum Retrovirol 1995; 8:273-278 [G]

104. Royall DR, Mahurin RK, Gray KF: Bedside assessment of executive cognitive impairment: the Executive Interview. J Am Geriatr Soc 1992; 40:1221-1226 [G]

105. Berghuis JP, Uldall KK, Lalonde B: Validity of two scales in identifying HIV-associated dementia. J Acquir Immune Defic Syndr 1999; 12:134-140 [G]

106. van Gorp WG, Satz P, Hinkin C, Selnes O, Miller EN, McArthur J, Cohen B, Paz D: Metacognition in HIV-1 seropositive asymptomatic individuals: self-ratings versus objective neuropsychological performance. J Clin Exp Neuropsychol 1991; 13:812-819 [C]

Treatment of Patients With HIV/AIDS 
107. Uldall KK, Harris VL, Lalonde B: Outcomes associated with delirium in acutely hospitalized acquired immune deficiency syndrome patients. Compr Psychiatry 2000; 41:88-91 [C]

108. Uldall KK, Ryan R, Berghuis JP, Harris VL: Association between delirium and death in AIDS patients. AIDS Patient Care STDS 2000; 14:95-100 [G]

109. Perkins DO, Leserman J, Stern RA, Baum SF, Liao D, Golden RN, Evans DL: Somatic symptoms and HIV infection: relationship to depressive symptoms and indicators of HIV disease. Am J Psychiatry 1995; 152:1776-1781 [C]

110. Brouillette MJ, Chouinard G, Lalonde R: Didanosine-induced mania in HIV infection (letter). Am J Psychiatry 1994; 151:1839-1840 [G]

111. Maxwell S, Scheftner WA, Kessler HA, Busch K: Manic syndrome associated with zidovudine treatment (letter). JAMA 1988; 259:3406-3407 [G]

112. Hoffman JA, Klein H, Clark DC, Boyd FT: The effect of entering drug treatment on involvement in HIV-related risk behaviors. Am J Drug Alcohol Abuse 1998; 24:259-284 [C]

113. American Psychiatric Association: Practice Guideline for Treatment of Patients With Substance Use Disorders: Alcohol, Cocaine, Opioids. Am J Psychiatry 1995; 152(Nov suppl):1-59 [G]

114. Pao M, Lyon M, D’Angelo LD, Schuman WB, Tipnis T, Mrazek DA: Psychiatric risk factors in HIV seropositive adolescents. Presented at the 1998 Annual Meeting of the American Academy of Child and Adolescent Psychiatry. Washington, DC, AACAP, 1998 [D]

115. Havens JF, Whitaker AH, Feldman JF, Ehrhardt AA: Psychiatric morbidity in school-age children with congenital human immunodeficiency virus infection: a pilot study. J Dev Behav Pediatr 1994; 15:S18-S25 [G]

116. Ramafedi G: The University of Minnesota Youth and AIDS Projects' Adolescent Early Intervention Program: a model to link HIV-seropositive youth with care. J Adolesc Health 1998; 23(2 suppl):115-121 [G]

117. Mattsson A: Long-term physical illness in childhood: a challenge to psychosocial adaptation. Pediatrics 1972; 50:801-811 [F]

118. Garrad SD, Richmond JB: Psychological aspects of the management of chronic diseases and handicapping conditions in childhood, in The Psychological Basis of Medical Practice. Edited by Lief HI, Lief VF, Lief NR. New York, Harper and Row, 1963, pp 370-403 [G]

119. Kotler D, Tierney AR, Wang J, Pierson RN: Magnitude of body cell mass depletion and the timing of death from wasting in AIDS. Am J Clin Nutr 1989; 50:44-47 [C]

120. Breitbart W, McDonald MV, Rosenfeld B, Monkman ND, Passik S: Fatigue in ambulatory AIDS patients. J Pain Symptom Manage 1998; 15:159-167 [C]

121. Ferrando S, Evans S, Goggin K, Sewell M, Fishman B, Rabkin J: Fatigue in HIV illness: relationship to depression, physical limitations, and disability. Psychosom Med 1998; 60: 759-764 [C]

122. Groopman JE: Fatigue in cancer and HIV/AIDS. Oncology (Huntingt) 1998; 12:335-344 $[\mathrm{G}]$

123. Breitbart W, McDonald MV, Rosenfeld B, Passik SD, Hewitt D, Thaler H, Portenoy RK: Pain in ambulatory AIDS patients, 1: pain characteristics and medical correlates. Pain 1996; 68:315-321 [A]

124. Laschinger SJ, Fothergill-Bourbonnais F: The experience of pain in persons with HIV/ AIDS. J Assoc Nurses AIDS Care 1999; 10:59-67 [G]

125. Singer EJ, Zorilla C, Fahy-Chandon B, Chi S, Syndulko K, Tourtellotte WW: Painful symptoms reported by ambulatory HIV-infected men in a longitudinal study. Pain 1993; 54:15-19[C]

126. Rabkin JG, Wagner GJ, Rabkin R: Testosterone therapy for human immunodeficiency virus-positive men with and without hypogonadism. J Clin Psychopharmacol 1999; 19: 19-27 [A] 
127. Centers for Disease Control and Prevention: HIV/AIDS Surveillance Report 1998; 10(2): $1-43[\mathrm{G}]$

128. HIV/AIDS among racial/ethnic minority men who have sex with men-United States. MMWR Morb Mortal Wkly Rep 2000; 1:4-11 [G]

129. Gonzales V, Washienko KM, Krone MR, Chapman LI, Arredondo EM, Huckeba HJ, Downer A: Sexual and drug-use risk factors for HIV and STDs: a comparison of women with and without bisexual experiences. Am J Public Health 1999; 89:1841-1846 [A]

130. CDC Division of HIV/AIDS Prevention: HIV/AIDS and US women who have sex with women (fact sheet). Atlanta, CDC National Center for HIV, STD and TB Prevention, $1999[\mathrm{G}]$

131. Carey MP: HIV and AIDS relative to other health, social, and relationship concerns among low-income urban women: a brief report. J Womens Health Gend Based Med 1999; 8: 657-661 [C]

132. Padian NS, Shiboski SC, Glass SO, Vittinghoff E: Heterosexual transmission of human immunodeficiency virus (HIV) in northern California: results from a ten-year study. Am J Epidemiol 1997;146:350-357 [C]

133. Farzadegan H, Hoover DR, Astemborski J, Lyles CM, Margolick JB, Markham RB, Quinn TC, Vlahov D: Sex differences in HIV-1 viral load and progression to AIDS. Lancet 1998; 352:1510-1514 [C]

134. Chung J, Magraw M: A group approach to psychosocial issues faced by HIV-positive women. Hosp Community Psychiatry 1992; 43:891-894 [G]

135. Gray JJ: The difficulties of women living with HIV infection. J Psychosoc Nurs Ment Health Serv 1999; 37:39-43 [G]

136. Thomas P: Americas: AIDS on the Rise Among Over-50s. BBC News Online, June 11, 1999.

http://news6.thdo.bbc.co.uk/hi/english/world/americas/newsid_366000/366702.stm [G]

137. Kasper SJ, Cavalieri TA: HIV-related medical issues in older people. Focus: A Guide to AIDS Research and Counseling 1999; 14:5-6 [G]

138. Stall R, Catania J: AIDS risk behaviors among late middle-aged and elderly Americans: the National AIDS Behavior Surveys. Arch Intern Med 1994; 154:57-63 [G]

139. Acuff C, Archambeault J, Greenberg B: Mental Health Care for People Living With or Affected by HIV/AIDS: A Practical Guide (SAMHSA Monograph).

www.mentalhealth.org/cmhs/HIVAIDS/mhcarehiv.htm [G]

140. CDC Division of HIV/AIDS Prevention: National data on HIV prevalence among disadvantaged youth in the 1990s (fact sheet). Atlanta, CDC National Center for HIV, STD and TB Prevention, 1998 [G]

141. Anderson JE, Wilson RW, Barker P, Doll L, Jones TS, Holtgrave D: Prevalence of sexual and drug-related HIV risk behaviors in the US adult population: results of the 1996 National Household Survey on Drug Abuse. J Acquir Immune Defic Syndr Hum Retrovirol 1999: 21:148-156 [C]

142. Crosby RA, Yarber WL, Meyerson B. Prevention strategies other than male condoms employed by low-income women to prevent HIV infection. Public Health Nursing 2000; 17:53-60 [D]

143. Heckman TG, Somlai AM, Sikkema KJ, Kelly JA, Franzoi SL: Psychosocial predictors of life satisfaction among persons living with HIV infection and AIDS. J Assoc Nurses AIDS Care 1997; 8:21-30 [C]

144. Beckett A, Shenson D: Suicide risk in patients with human immune deficiency virus infection and acquired immune deficiency syndrome. Harv Rev Psychiatry 1993; 1:27-35 [F]

145. Marzuk P, Tierney H, Tardiff K, Gross E, Morgan E, Hsu M, Mann J: Increased risk of suicide in persons with AIDS. JAMA 1988; 259:1333-1337 [D] 
146. Kizer KW, Green M, Perkins CI, Doebbert G, Hughes MJ: AIDS and suicide in California (letter). JAMA 1998; 260:1881 [D]

147. Cote T, Biggar R, Dannenberg A: Risk of suicide among persons with AIDS: a national assessment. JAMA 1992; 268:2066-2068 [D]

148. Marzuk PM, Tardiff K, Leon AC, Hirsch CS, Hartwell N, Potera L, Iqbal MI: HIV seroprevalence among suicide victims in New York City, 1991-1993. Am J Psychiatry 1997; 154:1720-1725 [D]

149. McDaniel JS, Purcell DW, D'Augelli AR: The relationship between sexual orientation and risk for suicide: research findings and future directions for research and prevention. Suicide Life Threat Behav 2001; 31(suppl):84-105 [F]

150. Dannenberg AI, McNeil JG, Brundage JF, Brookmeyer R: Suicide and HIV infection: mortality follow-up of 4,147 HIV-seropositive military service applicants. JAMA 1996; 276:1743-1746 [C]

151. van Haastrecht HJ, Mientjes GH, van den Hoek AJ, Coutinho RA: Death from suicide and overdose among drug injectors after disclosure of first HIV test result. AIDS 1994; 8: $1721-1725[\mathrm{C}]$

152. Westermeyer J, Seppala M, Gasow S, Carlson G: AIDS-related illness and AIDS risk in male homo/bisexual substance abusers: case reports and clinical issues. Am J Drug Alcohol Abuse 1989; 15:443-461 [G]

153. Lester D: Sexual versus psychiatric predictors of suicide in men with AIDS-related illnesses. Am J Drug Alcohol Abuse 1992; 19:139-140 [D]

154. Kelly B, Raphael B, Judd F, Perdices M, Kernutt G, Burnett P, Dunne M, Burrows G: Suicidal ideation, suicide attempts, and HIV infection. Psychosomatics 1998; 39:405-415 [D]

155. Goldblum P, Moulton J: HIV disease and suicide, in Face to Face: A Guide to AIDS Counseling. Edited by Dilley JW, Pies C, Helquist M. Berkeley, Calif, Celestial Arts, 1989, pp 152-164 [G]

156. Houston-Vega M, Ward J: Suicide assessment and intervention with persons infected with HIV, in HIV and Community Mental Healthcare. Edited by Knox M, Sparks C. Baltimore, Johns Hopkins University Press, 1998, pp 178-194 [G]

157. Mayne TJ, Acree M, Chesney MA, Folkman S: HIV sexual risk behavior following bereavement in gay men. Health Psychol 1998; 17:403-411 [C]

158. Rosengard C, Folkman S: Suicidal ideation, bereavement, HIV serostatus and psychosocial variables in partners of men with AIDS. AIDS Care 1997; 9:373-384 [C]

159. Martin JL, Dean L: Effects of AIDS-related bereavement and HIV-related illness on psychological distress among gay men: a 7-year longitudinal study, 1985-1991. J Consult Clin Psychol 1993; 61:94-103 [C]

160. Sherr L, Hedge B, Steinhart K, Davey T, Petrack J: Unique patterns of bereavement in HIV: implications for counselling. Genitourin Med 1992; 68:378-381 [G]

161. Rotheram-Borus MJ, Draimin BH, Reid HM, Murphy DA: The impact of illness disclosure and custody plans on adolescents whose parents live with AIDS. AIDS 1997; 11:1159$1164[\mathrm{G}]$

162. Rotheram-Borus MJ, Stein JA: Problem behavior of adolescents whose parents are living with AIDS. Am J Orthopsychiatry 1999; 69:228-239 [G]

163. Neugebauer R, Rabkin JG, Williams JB, Remien RH, Goetz R, Gorman JM: Bereavement reactions among homosexual men experiencing multiple losses in the AIDS epidemic. Am J Psychiatry 1992; 149:1374-1379 [D]

164. Goodkin K, Blaney N, Tuttle R, Nelson R, Baidewicz T, Kumar M, Fletcher M, Leeds B, Feaster D: Bereavement and HIV infection. Int Rev Psychiatry 1996; 8:201-216 [F]

165. Kemeny ME, Weiner H, Taylor SE, Schneider S, Visscher B, Fahey JL: Repeated bereavement, depressed mood, and immune parameters in HIV seropositive and seronegative gay men. Health Psychol 1994; 13:14-24 [D] 
166. Bartrop RW, Luckhurst E, Lazarus L, Kiloh LG, Penny R: Depressed lymphocyte function after bereavement. Lancet 1977; 8016:834-836 [C]

167. Irwin M, Daniels M, Smith TL, Bloom E, Weiner H: Impaired natural killer cell activity during bereavement. Brain Behav Immun 1987; 1:98-104 [D]

168. Goodkin K, Feaster DJ, Tuttle R, Blaney NT, Kumar M, Baum MK, Shapshak P, Fletcher MA: Bereavement is associated with time-dependent decrements in cellular immune function in asymptomatic human immunodeficiency virus type 1-seropositive homosexual men. Clin Diagn Lab Immunol 1996; 3:109-118 [C]

169. Sikkema KJ, Kalichman SC, Kelly JA, Koob JJ: Group intervention to improve coping with AIDS-related bereavement: model development and an illustrative clinical example. AIDS Care 1995; 7:463-475 [G]

170. Goodkin K, Blaney NT, Feaster DJ, Baldewicz T, Burkhalter JE, Leeds B: A randomized controlled clinical trial of a bereavement support group intervention in human immunodeficiency virus type 1-seropositive and -seronegative homosexual men. Arch Gen Psychiatry 1999; 56:52-59 [A]

171. Golding M, Perkins DO: Personality disorder in HIV infection. Int Rev Psychiatry 1996; $8: 253[\mathrm{~F}]$

172. Jacobsberg L, Frances A, Perry S: Axis II diagnoses among volunteers for HIV testing and counseling. Am J Psychiatry 1995; 152:1222-1224 [G]

173. Treisman GJ, Lyketsos CG, Fishman M, Hanson AL, Rosenblatt A, McHugh PR: Psychiatric care for patients with HIV infection: the varying perspectives. Psychosomatics 1993; 34:432-439 [F]

174. Perkins DO, Davidson EJ, Leserman J, Liao D, Evans DL: Personality disorder in patients infected with HIV: a controlled study with implications for clinical care. Am J Psychiatry 1993; 150:309-315 [C]

175. Johnson JG, Williams JB, Rabkin JG, Goetz RR, Remien RH: Axis I psychiatric symptoms associated with HIV infection and personality disorder. Am J Psychiatry 1995; 152:551-554 [C]

176. Brooner RK, Bigelow GE, Strain E, Schmidt CW: Intravenous drug abusers with antisocial personality disorder: increased HIV risk behavior. Drug Alcohol Depend 1990; 26:39-44 [G]

177. Johnson JG, Williams JB, Goetz RR, Rabkin JG, Remien RH, Lipsitz JD, Gorman JM: Personality disorders predict onset of axis I disorders and impaired functioning among homosexual men with and at risk of HIV infection. Arch Gen Psychiatry 1996; 53:350$357[\mathrm{C}]$

178. Goodkin K, Fuchs I, Feaster D, Leeka J, Rishel DD: Life stressors and coping style are associated with immune measures in HIV-1 infection-a preliminary report. Int J Psychiatry Med 1992; 22:155-172 [G]

179. Goodkin K, Mulder CL, Blaney NT, Ironson G, Kumar M, Fletcher MA: Psychoneuroimmunology and human immunodeficiency virus type 1 infection revisited. Arch Gen Psychiatry 1994; 51:246-248 [G]

180. Kemeny ME, Weiner H, Duran R, Taylor SE, Visscher B, Fahey JL: Immune system changes after the death of a partner in HIV-positive gay men. Psychosom Med 1995; 57:547-554 [D]

181. Evans DL, Leserman J, Perkins DO, Stern RA, Murphy C, Tamul K, Liao D, van der Horst CM, Hall CD, Folds JD: Stress-associated reductions of cytotoxic T lymphocytes and natural killer cells in asymptomatic HIV infection. Am J Psychiatry 1995; 152:543-550 [C]

182. Leserman J, Petitto JM, Perkins DO, Folds JD, Golden RN, Evans DL: Severe stress, depressive symptoms, and changes in lymphocyte subsets in human immunodeficiency virus-infected men. A 2-year follow-up study. Arch Gen Psychiatry 1997; 54:279-285 [C]

183. Leserman J, Jackson ED, Petitto JM, Golden RN, Silva SG, Perkins DO, Cai J, Folds JD, Evans DL: Progression to AIDS: the effects of stress, depressive symptoms, and social support. Psychosom Med 1999; 61:397-406 [C] 
184. Rabkin JG, Williams JB, Remien RH, Goetz R, Kertzner R, Gorman JM: Depression, distress, lymphocyte subsets, and human immunodeficiency virus symptoms on two occasions in HIV-positive homosexual men. Arch Gen Psychiatry 1991; 48:111-119 [C]

185. Perry S, Fishman B, Jacobsberg L: Stress and HIV infection. Am J Psychiatry 1992; 149: 416-417 [G]

186. Burack JH, Barrett DC, Stall RD, Chesney MA, Ekstrand ML, Coates TJ: Depressive symptoms and CD4 lymphocyte decline among HIV-infected men. JAMA 1993; 270: 2568-2573 [C]

187. Lyketsos CG, Hoover DR, Guccione M, Senterfitt W, Dew MA, Wesch J, VanRaden MJ, Treisman GJ, Morgenstern H: Depressive symptoms as predictors of medical outcomes in HIV infection: Multicenter AIDS Cohort Study. JAMA 1993; 270:2563-2567 [C]

188. Antoni MH, Baggett L, Ironson G, LaPerriere A, August S, Klimas N, Schneiderman N, Fletcher MA: Cognitive-behavioral stress management intervention buffers distress responses and immunologic changes following notification of $\mathrm{HIV}-1$ seropositivity. J Consult Clin Psychol 1991; 59:906-915 [C]

189. Goodkin K, Feaster DJ, Asthana D, Blaney NT, Kumar M, Baldewicz T, Tuttle RS, Maher KJ, Baum MK, Shapshak P, Fletcher MA: A bereavement support group intervention is longitudinally associated with salutary effects on the CD4 cell count and number of physician visits. Clin Diagn Lab Immunol 1998; 5:382-391 [A]

190. Rabkin JG, Rabkin R, Harrison W, Wagner G: Effect of imipramine on mood and enumerative measures of immune status in depressed patients with HIV illness. Am J Psychiatry 1994; 151:516-523 [A]

191. Eisenberg DM, Kessler RC, Foster C, Norlock FE, Calkins DR, Delbanco TL: Unconventional medicine in the United States: prevalence, costs, and patterns of use. N Engl J Med 1993; 328:246-252 [G]

192. Eisenberg DM, Davis RB, Ettner SL, Appel S, Wilkey S, Van Rompay M, Kessler RC: Trends in alternative medicine use in the United States, 1990-1997: results of a follow-up national survey. JAMA 1998; 280:1569-1575 [G]

193. Fairfield KM, Eisenberg DM, Davis RB, Libman H, Phillips RS: Patterns of use, expenditures, and perceived efficacy of complementary and alternative therapies in HIV-infected patients. Arch Intern Med 1998; 158:2257-2264 [D]

194. Ostrow MJ, Cornelisse PG, Heath KV, Craib KJ, Schechter MT, O'Shaughnessy M, Montaner JS, Hogg RS: Determinants of complementary therapy use in HIV-infected individuals receiving antiretroviral or anti-opportunistic agents. J Acquir Immune Defic Syndr Hum Retrovirol 1997; 15:115-120 [G]

195. Singh N, Squier C, Sivek C, Nguyen M, Wagener M, Yu VL: Determinants of nontraditional therapy use in patients with HIV infection: a prospective study. Arch Intern Med 1996; 156:197-201 [C]

196. Anderson W, O’Connor B, MacGregor R, Schwartz J: Patient use and assessment of conventional and alternative therapies for HIV infection and AIDS. AIDS 1993; 7:561-565 [F]

197. Calabrese C, Wenner C, Reeves C, Turet P, Standish L: Treatment of human immunodeficiency virus-positive patients with complementary and alternative medicine: a survey of practitioners. J Altern Complement Med 1998; 4:281-287 [G]

198. Macintyre R, Holzemer W: Complementary and alternative medicine and HIV/AIDS, part II: selected literature review. J Assoc Nurses AIDS Care 1997; 8:25-38 [F]

199. Greene KB, Berger J, Reeves C, Moffat A, Standish LJ, Calabrese C: Most frequently used alternative and complementary therapies and activities by participants in the AMCOA Study. J Assoc Nurses AIDS Care 1999; 10:60-73 [E]

200. Standish L, Calabrese C, Reeves C, Polissar N, Bain S, O’Donnell T: A scientific plan for the evaluation of alternative medicine in the treatment of HIV/AIDS. Altern Ther Health Med 1997; 3:58-67 [G] 
201. Pincus SH, Schoenbaum EE, Webber M: A seroprevalence survey for human immunodeficiency virus antibody in mentally retarded adults. NY State J Med 1990; 90:139-142 [G]

202. Lohiya GS: Human immunodeficiency virus type-1 antibody in 6,703 institutionalized mentally retarded clients: an unlinked serosurvey at seven California developmental centers. AIDS Res Hum Retroviruses 1993; 9:247-249 [G]

203. Altice FL, Mostashari F, Selwyn PA, Checko PJ, Singh R, Tanguay S, Blanchette EA: Predictors of HIV infection among newly sentenced male prisoners. J Acquir Immune Defic Syndr Hum Retrovirol 1998; 18:444-453 [C]

204. Behrendt C, Kendig N, Dambita C, Horman J, Lawlor J, Vlahov D: Voluntary testing for human immunodeficiency virus (HIV) in a prison population with a high prevalence of HIV. Am J Epidemiol 1994; 139:918-926 [E]

205. Smith PF, Mikl J, Truman BI, Lessner L, Lehman JS, Stevens RW, Lord EA, Broaddus RK, Morse DL: HIV infection among women entering the New York State correctional system. Am J Public Health Suppl 1991; 81:35-40 [G]

206. Grinstead OA, Zack B, Faigeles B: Collaborative research to prevent HIV among male prison inmates and their female partners. Health Educ Behav 1999; 26:225-238 [G]

207. Silverman DC: Psychosocial impact of HIV-related caregiving on health providers: a review and recommendations for the role of psychiatry. Am J Psychiatry 1993; 150:705-712 [F]

208. McDaniel JS, Farber EW, Summerville MB: Mental health care providers working with HIV: avoiding stress and burnout, in Textbook of Homosexuality and Mental Health. Edited by Cabaj RP, Stein TS. Washington, DC, American Psychiatric Press, 1996, pp 839-858 [G]

209. Catalan J, Burgess A, Pergami A, Hulme N, Gazzard B, Phillips R: The psychological impact on staff of caring for people with serious diseases - the case of HIV infection and oncology. J Psychosom Res 1996; 40:425-435 [D]

210. Barbour RS: The impact of working with people with HIV/AIDS: a review of the literature. Soc Sci Med 1994; 39:221-232 [F]

211. Frost JC: Support groups for medical caregivers of people with HIV diseases. Group 1994; $18: 141-153[\mathrm{G}]$

212. Wainberg ML: 52nd American Group Psychotherapy Association Annual Meeting, Atlanta $1995[\mathrm{G}]$

213. Aruffo JE, Thompson R, McDaniel JS, Sacco J, Herman R, Kaplan M, Andriote J, Washington R, Eversole T: Training programs for staff, in AIDS and People With Severe Mental Illness: A Handbook for Mental Health Professionals. Edited by Cournos F, Bakalar N. New Haven, Conn, Yale University Press, 1996, pp 201-224 [G]

214. Brown LK, Stermock AC, Ford HH, Geary M: Emotional reactions of haemophilia health care providers. Haemophilia 1999; 5:127-131 [G]

215. Chung JY, Suarez AP, Zarin DA, Pincus HA: Psychiatric patients and HIV. Psychiatr Serv 1999; 50:487 [G]

216. Epstein RM, Morse DS, Frankel RM, Frarey L, Anderson K, Beckman HB: Awkward moments in patient-physician communication about HIV risk. Ann Intern Med 1998; 128 : $435-442[\mathrm{C}]$

217. Bindman AB, Osmond D, Hecht FM, Lehman JS, Vranizan K, Keane D, Reingold A (Multistate Evaluation of Surveillance of HIV [MESH] Study Group): Multistate evaluation of anonymous HIV testing and access to medical care. JAMA 1998; 16:1416-1420 [D]

218. Nakashima AK, Horsley R, Frey RL, Sweeney PA, Weber JT, Fleming PL: Effect of HIV reporting by name on use of HIV testing in publicly funded counseling and testing programs. JAMA 1998; 16:1421-1426 [G]

219. Osmond DH, Bindman AB, Vranizan K, Lehman JS, Hecht FM, Keane D, Reingold A (Multistate Evaluation of Surveillance for HIV Study Group): Name-based surveillance and public health interventions for persons with HIV infection. Ann Intern Med 1999; 131: $775-779[\mathrm{G}]$ 
220. Branson BM: Home sample collection tests for HIV infection. JAMA 1998; 280:1699$1701[\mathrm{G}]$

221. Coates TJ: Strategies for modifying sexual behavior for primary and secondary prevention of HIV disease. J Consult Clin Psychol 1990; 58:57-69 [F]

222. Guenther-Grey C, Noroian D, Fonseka J, Higgins D: Developing community networks to deliver HIV prevention interventions. Public Health Rep 1996; 111(suppl 1):41-49 [G]

223. Cabaj RP, Purcell DW (eds): On the Road to Same-Sex Marriage: A Supportive Guide to Psychological, Political, and Legal Issues. San Francisco, Jossey-Bass, 1998 [G]

224. Joint United Nations Programme on HIV/AIDS: Sexual Behavioural Change for HIV: Where Have Theories Taken Us? Geneva, UNAIDS, 1999 [F]

225. HIV/AIDS Prevention Research Synthesis Project: Compendium of HIV Prevention Interventions With Evidence of Effectiveness. Atlanta, CDC, 1999 [G]

226. Higgins DL, Galavotti C, O'Reilly KR, Schnell DJ, Moore M, Rugg DL, Johnson R: Evidence for the effects of HIV antibody counseling and testing on risk behaviors. JAMA 1991; 266:2419-2429 [F]

227. Wolitski RJ, MacGowan RJ, Higgins DL, Jorgensen CM: The effects of HIV counseling and testing on risk-related practices and help-seeking behavior. AIDS Educ Prev 1997; 9(3 suppl):52-67 [F]

228. Weinhardt LS, Carey MP, Johnson BT, Bickham NL: Effects of HIV counseling and testing on sexual risk behavior: a meta-analytic review of published research, 1985-1997. Am J Public Health 1999; 89:1397-1405 [E]

229. Prochaska JO, DiClemente CC: Stages of change in the modification of problem behaviors. Prog Behav Modif 1992; 28:183-218 [F]

230. Azjen I, Fishbeign M: Understanding Attitudes and Predicting Behavior. Englewood Cliffs, NJ, Prentice Hall, 1980 [G]

231. Fisher JD, Fisher WA: Changing AIDS-risk behavior. Psychol Bull 1992; 111:455-474 [G]

232. Kalichman SC, Hospers HJ: Efficacy of behavioral-skills enhancement HIV risk-reduction interventions in community settings. AIDS 1997; 11(suppl A):S191-S199 [F]

233. National Institute of Mental Health (NIMH) Multisite HIV Prevention Trial Group: The NIMH Multisite HIV Prevention Trial: reducing HIV sexual risk behavior. Science 1998; 280:1889-1894 [A]

234. Kamb ML, Fishbein M, Douglas JM Jr, Rhodes F, Rogers J, Bolan G, Zenilman J, Hoxworth T, Malotte CK, Iatesta M, Kent C, Lentz A, Graziano S, Byers RH, Peterman TA (Project RESPECT Study Group): Efficacy of risk-reduction counseling to prevent human immunodeficiency virus and sexually transmitted diseases: a randomized controlled trial. JAMA 1998; 280:1161-1167 [A]

235. Kelly JA, McAuliffe TL, Sikkema KJ, Murphy DA, Somlai AM, Mulry G, Miller JG, Stevenson LY, Fernandez MI: Reduction in risk behavior among adults with severe mental illness who learned to advocate for HIV prevention. Psychiatr Serv 1997; 48:1283-1288 [A]

236. Zule WA, Desmond DP: An ethnographic comparison of HIV risk behaviors among heroin and methamphetamine injectors. Am J Drug Alcohol Abuse 1999; 25:1-23 [D]

237. Des Jarlais D, Friedman SR: HIV epidemiology and interventions among injecting drug users. Int J STD AIDS 1996; 7(suppl 2):57-61 [F]

238. Coyle SL, Needle RH, Normand J: Outreach-based HIV prevention for injecting drug users: a review of published outcome data. Public Health Rep 1998; 113(suppl 1):19-30 [F]

239. Strathdee SA, Celentano DD, Shah N, Lyles C, Stambolis VA, Macalino G, Nelson K, Vlahov D: Needle-exchange attendance and health care utilization promote entry into detoxification. J Urban Health 1999; 76:448-460 [C]

240. Viahov D, Junge B: The role of needle exchange programs in HIV prevention. Public Health Rep 1998; 113(suppl 1):75-80 [F] 
241. Hurley SF, Jolley DJ, Kaldor JM: Effectiveness of needle-exchange programmes for prevention of HIV infection. Lancet 1997; 349:1797-1800 [E]

242. CDC Division of HIV/AIDS Prevention: Status of Perinatal HIV Prevention: U.S. Declines Continue (fact sheet). Atlanta, CDC National Center for HIV, STD and TB Prevention, $1999[\mathrm{G}]$

243. Lindegren ML, Byers RH Jr, Thomas P, Davis SF, Caldwell B, Rogers M, Gwinn M, Ward JW, Fleming PL: Trends in perinatal transmission of HIV/AIDS in the United States. JAMA 1999; 282:531-538 [G]

244. Riley LE, Greene MF: Elective cesarean delivery to reduce the transmission of HIV. N Engl J Med 1999; 340:1032-1033 [G]

245. Centers for Disease Control and Prevention: AIDS among children-United States, 1996. MMWR Morb Mortal Wkly Rep 1996; 45:1005-1010 [G]

246. Brady M, McGrath N, Brouwers P, Gelber R, Fowler M, Yogev R, Hutton N, Bryson YJ, Mitchell CD, Fikrig S, Borkowsky W, Jimenez E, McSherry G, Rubinstein A, Wilfert CM, McIntosh K, Elkins MM, Weintrub PS (Pediatric AIDS Clinical Trials Group 128): Randomized study of the tolerance and efficacy of high- versus low-dose zidovudine in human immunodeficiency virus-infected children with mild to moderate symptoms. J Infect Dis 1996; 173:1097-1106 [B]

247. Mofenson LM, Lambert JS, Stiehm ER, Bethel J, Meyer WA III, Whitehouse J, Moye J, Jr, Reichelderfer P, Harris DR, Fowler MG, Mathieson BJ, Nemo GJ (Pediatric AIDS Clinical Trials Group Study 185 Team): Risk factors for perinatal transmission of human immunodeficiency virus type 1 in women treated with zidovudine. N Engl J Med 1999; 341:385-393 [A]

248. Garcia PM, Kalish LA, Pitt J, Minkoff H, Quinn TC, Burchett SK, Kornegay J, Jackson B, Moye J, Hanson C, Zorrilla C, Lew JF (Women and Infants Transmission Study Group): Maternal levels of plasma human immunodeficiency virus type I RNA and the risk of perinatal transmission. N Engl J Med 1999; 341:394-402 [G]

249. Sandberg JA, Slikker W Jr: Developmental pharmacology and toxicology of anti-HIV therapeutic agents: dideonucleosides. FASEB J 1995; 9:1157-1163 [G]

250. Centers for Disease Control and Prevention: Administration of zidovudine during late pregnancy and delivery to prevent perinatal HIV transmission-Thailand, 1996-1998. MMWR Morb Mortal Wkly Rep 1998; 47:151-154 [G]

251. Leroy V, Dabis F: Reduction of mother-child transmission of HIV infection in Africa: from clinical research to public health programs. Med Trop (Mars) 1999; 59:456-464 [F]

252. Public Health Service Task Force recommendations for the use of antiretroviral drugs in pregnant women infected with HIV-1 for maternal health and for reducing perinatal HIV1 transmission in the United States. MMWR Morb Mortal Wkly Rep 1998; 47(RR-2):130; corrections, 47(14):287, 47(15):315 [G]

253. Lorenzi P, Spicher VM, Laubereau B, Hirschel B, Kind C, Rudin C, Irion O, Kaiser L: Antiretroviral therapies in pregnancy: maternal, fetal and neonatal effects: Swiss HIV Cohort Study, the Swiss Collaborative HIV and Pregnancy Study, and the Swiss Neonatal HIV Study. AIDS 1998; 12:F241-F247 [C]

254. Bell DM: Occupational risk of human immunodeficiency virus infection in healthcare workers: an overview. Am J Med 1997; 102(suppl 5B):9-15 [F]

255. Cardo DM, Culver DH, Ciesielski CA, Srivastava PU, Marcus R, Abiteboul D, Heptonstall J, Ippolito G, Lot F, McKibben PS, Bell DM: A case-control study of HIV seroconversion in health care workers after percutaneous exposure. N Engl J Med 1997; 337:1485-1490 [D]

256. Kotranski L, Semaan S, Collier K, Lauby J, Halbert J, Feighan K: Effectiveness of an HIV risk reduction counseling intervention for out-of-treatment drug users. AIDS Educ Prev $1998 ; 10: 19-33[\mathrm{~A}]$ 
257. Hoff RA, Beam-Goulet J, Rosenheck RA: Mental disorder as a risk factor for human immunodeficiency virus infection in a sample of veterans. J Nerv Ment Dis 1997; 185:556$560[\mathrm{G}]$

258. Polonsky S, Kerr S, Harris B, Gaiter J, Fichtner RR, Kennedy MG: HIV prevention in prisons and jails: obstacles and opportunities. Public Health Rep 1994; 109:615-625 [G]

259. Inciardi JA: HIV risk reduction and service delivery strategies in criminal justice settings. J Subst Abuse Treat 1996; 13:421-428 [F]

260. Katz RC, Watts C, Santman J: AIDS knowledge and high risk behaviors in the chronic mentally ill. Community Ment Health J 1994; 4:395-402 [G]

261. Carey MP, Carey KB, Kalichman SC: Risk for human immunodeficiency virus (HIV) infection among persons with severe mental illnesses. Clin Psychol Rev 1997; 17:271-291 [F]

262. Kalichman SC, Sikkema KJ, Kelly JA, Bulto M: Use of a brief behavioral skills intervention to prevent HIV infection among chronic mentally ill adults. Psychiatr Serv 1995; 46:275$280[\mathrm{G}]$

263. Katz RC, Westerman C, Beauchamp K, Clay C: Effects of AIDS counseling and risk reduction training on the chronic mentally ill. AIDS Educ Prev 1996; 8:457-463 [A]

264. Zierler S, Witbeck B, Mayer K: Sexual violence against women living with or at risk for HIV infection. Am J Prev Med 1996; 12:304-310 [C]

265. Lenderking WR, Wold C, Mayer KH, Goldstein R, Losina E, Seage GR III: Childhood sexual abuse among homosexual men: prevalence and association with unsafe sex. J Gen Intern Med 1997; 12:250-253 [G]

266. Thompson NJ, Potter JS, Sanderson CA, Maibach EW: The relationship of sexual abuse and HIV risk behaviors among heterosexual adult female STD patients. Child Abuse Negl 1997; 21:149-156 [G]

267. Kalichman SC, Williams EA, Cherry C, Belcher L, Nachimson D: Sexual coercion, domestic violence, and negotiating condom use among low-income African American women. J Womens Health 1998; 7:371-378 [G]

268. Lindegren ML, Hanson IC, Hammett TA, Beil J, Fleming PL, Ward JW: Sexual abuse of children: intersection with the HIV epidemic. Pediatrics 1998; 102:E46 [G]

269. Brown LK, Kessel SM, Lourie KJ, Ford HH, Lipsitt LP: Influence of sexual abuse on HIVrelated attitudes and behaviors in adolescent psychiatric inpatients. J Am Acad Child Adolesc Psychiatry 1997; 36:316-322 [G]

270. Fleishman JA, Hsia DC, Hellinger FJ: Correlates of medical service utilization among people with HIV infection. Health Serv Res 1994; 29:527-548 [C]

271. AIDS mortality rates lower at sites with HIV experience. AIDS Alert, Nov 1999, p 129 [G]

272. Chaisson RE, Keruly JC, Moore RD: Race, sex, drug use, and progression of human immunodeficiency virus disease. N Engl J Med 1995; 333:751-756 [G]

273. Shapiro MF, Morton SC, McCaffrey DF, Senterfitt JW, Fleishman JA, Perlman JF, Athey LA, Keesey JW, Goldman DP, Berry SH, Bozzette SA: Variations in the care of HIV-infected adults in the United States: results from the HIV Cost and Services Utilization Study. JAMA 1999; 281:2305-2315 [C]

274. Kelly JA, Murphy DA, Bahr GR, Koob JJ, Morgan MG, Kalichman SC, Stevenson LY, Brasfield TL, Bernstein BM, St Lawrence JS: Factors associated with severity of depression and high-risk sexual behavior among persons diagnosed with human immunodeficiency virus (HIV) infection. Health Psychol 1993; 12:215-219 [D]

275. Friedmann PD, Alexander JA, Jin L, D'Aunno TA: On-site primary care and mental health services in outpatient drug abuse treatment units. J Behav Health Serv Res 1999; 26:80$94[\mathrm{~F}]$

276. Gomez MF, Klein DA, Sand S, Marconi M, O'Dowd MA: Delivering mental health care to HIV-positive individuals: a comparison of two models. Psychosomatics 1999; 40:321$324[\mathrm{G}]$ 
277. Ayuso JL: Use of psychotropic drugs in patients with HIV infection. Drugs 1994; 47:599$610[\mathrm{G}]$

278. Tashima K, Bose T, Gormley J, Sousa H, Flanigan TP: The potential impact of efavirenz on methadone maintenance (abstract). Conference on Retroviruses and Opportunistic Infections 1999; 6 [G]

279. Morse GD, Fischl MA, Shelton MJ, Cox SR, Driver M, DeRemer M, Freimuth WW: Single-dose pharmacokinetics of delavirdine mesylate and didanosine in patients with human immunodeficiency virus infection. Antimicrob Agents Chemother 1997; 41:169$174[\mathrm{~A}]$

280. Beertz R, Cao G, Cavanaugh JH, Hsu A, Granneman GR, Leonard JM: Effect of ritonavir on the pharmacokinetics of desipramine (abstract 1201), in Abstracts of the 11th International Conference on AIDS. Vancouver, BC, International AIDS Society, 1996 [G]

281. von Moltke LL, Greenblatt DJ, Duan SX, Daily JP, Harmatz JS, Shader RI: Inhibition of desipramine hydroxylation (Cytochrome P450-2D6) in vitro by quinidine and by viral protease inhibitors: relation to drug interactions in vivo. J Pharm Sci 1998; 87:1184-1189 [G]

282. Merry C, Mulcahy F, Barry M, Gibbons S, Back D: Saquinavir interaction with midazolam; pharmacokinetic considerations when prescribing protease inhibitors for patients with HIV disease (letter). AIDS 1997; 11:268-269 [G]

283. Henry JA, Hill IR: Fatal interaction between ritonavir and MDMA. Lancet 1998; 352: $1751-1752[\mathrm{G}]$

284. Hintz S, Kuck J, Peterkin J, Volk D, Zisook S: Depression in the context of human immunodeficiency virus infection: implications for treatment. J Clin Psychiatry 1990; 51: 497-501 [G]

285. Ferrando S, Goldman J, Charness W: Selective serotonin reuptake inhibitor treatment of depression in symptomatic HIV infection and AIDS. Gen Hosp Psychiatry 1997; 19:89$97[\mathrm{~B}]$

286. Wagner G, Rabkin J, Rabkin R: A comparative analysis of standard and alternative antidepressants in the treatment of human immunodeficiency virus patients. Compr Psychiatry 1996; 37:402-408 [A]

287. Elliott AJ, Uldall KK, Bergam K, Russo J, Claypoole K, Roy-Byrne PP: Randomized, placebo-controlled trial of paroxetine versus imipramine in depressed HIV-positive outpatients. Am J Psychiatry 1998; 155:367-372 [A]

288. Elliott AJ, Russo J, Bergam K, Claypoole K, Uldall KK, Roy-Byrne PP: Antidepressant efficacy in HIV-seropositive outpatients with major depressive disorder: an open trial of nefazodone. J Clin Psychiatry 1999; 60:226-231 [B]

289. Rabkin JG, Wagner GJ, Rabkin R: Fluoxetine treatment for depression in patients with HIV and AIDS: a randomized, placebo-controlled trial. Am J Psychiatry 1999; 156:101$107[\mathrm{~A}]$

290. Zisook S, Peterkin J, Goggin KJ, Sledge P, Atkinson JH, Grant I (HIV Neurobehavioral Research Center Group): Treatment of major depression in HIV-seropositive men. J Clin Psychiatry 1998; 59:217-224 [A]

291. Cornblath DR, McArthur JC: Predominantly sensory neuropathy in patients with AIDS and AIDS-related complex. Neurology 1988; 38:794-796 [C]

292. Vitiello B, Stover ES: Psychopharmacology in HIV-positive patients: research perspectives. Psychopharmacol Bull 1996; 32:293-297 [G]

293. Rabkin J, Rabkin R, Wagner G: Effects of fluoxetine on mood and immune status in depressed patients with HIV illness. J Clin Psychiatry 1994; 55:92-97 [B]

294. Schwartz JA, McDaniel JS: Double-blind comparison of fluoxetine and desipramine in the treatment of depressed women with advanced HIV disease: a pilot study. Depress Anxiety 1999; 9:70-74 [A] 
295. Grassi B: Notes on the use of fluvoxamine as treatment of depression in HIV-1 infected subjects. Pharmacopsychiatry 1995; 28:93-94 [B]

296. Gillenwater DR, McDaniel JS: Rational psychopharmacology in patients with HIV infection and AIDS. Psychiatr Annals (in press) [F]

297. Levin GM, Nelson LA, Devane CL, Preston SL, Carson SW, Eisele G: Venlafaxine and indinavir: results of a pharmacokinetic interaction study (abstract 661), in Abstracts of the 39th Interscience Conference on Antimicrobial Agents and Chemotherapy. Washington, DC, American Society for Microbiology, 1999 [G]

298. Scurlock HJ, Singh AN, Catalan J: Atypical antipsychotic drugs in the treatment of manic syndromes in patients with HIV-1 infection. J Psychopharmacol 1995; 9:151-154 [G]

299. Hriso E, Kuhn T, Masdeu JC, Grundman M: Extrapyramidal symptoms due to dopamineblocking agents in patients with AIDS encephalopathy. Am J Psychiatry 1991; 148:1558$1561[\mathrm{D}]$

300. Breitbart W, Marotta RF, Call P: AIDS and neuroleptic malignant syndrome. Lancet 1988; 2:1488-1489 [G]

301. Maccario M, Scharre DW: HIV and acute onset of psychosis (letter). Lancet 1987; 2:342 [G]

302. Edelstein H, Knight RT: Severe parkinsonism in two AIDS patients taking prochlorperazine (letter). Lancet 1987; 2:341-342 [G]

303. Kieburtz K, Epstein L, Gelbard H, Greenamyre J: Excitotoxicity and dopaminergic dysfunction in the acquired immunodeficiency syndrome dementia complex. Arch Neurol 1991; 48:1281-1284 [G]

304. Swenson JR, Erman M, Labelle J, Dimsdale JE: Extrapyramidal reactions: neuropsychiatric mimics in patients with AIDS. Gen Hosp Psychiatry 1989; 11:248-253 [G]

305. Hollander H, Golden J, Mendelson T, Cortland D: Extrapyramidal symptoms in AIDS patients given low-dose metoclopramide or chlorpromazine (letter). Lancet 1985; 2:1186 [G]

306. Vogel-Scibilia S, Mulsant BH, Keshavan MS: HIV infection presenting as psychosis: a critique. Acta Psychiatr Scand 1988; 78:652-656 [G]

307. Rosebush P, Stewart T: A prospective analysis of 24 episodes of neuroleptic malignant syndrome. Am J Psychiatry 1989; 146:717-725 [B]

308. Gabel R, Barnard N, Norko M, O'Connell R: AIDS presenting as mania. Compr Psychiatry 1986; 27:251-254 [G]

309. Ferrando S, Eisendrath S: Adverse neuropsychiatric effects of dopamine antagonist medications: misdiagnosis in the medical setting. Psychosomatics 1991; 32:426-432 [C]

310. Belzie LR: Risperidone for AIDS-associated dementia—a case series. AIDS Patient Care and STDs 1996; 10:246-249 [G]

311. Shedlack KJ, Soldato-Couture C, Swanson CL Jr: Rapidly progressive tardive dyskinesia in AIDS (letter). Biol Psychiatry 1994; 35:147-148 [G]

312. Fernandez F, Joel L: The use of molindone in the treatment of psychotic and delirious patients infected with the human immunodeficiency virus: case reports. Gen Hosp Psychiatry 1993; $15: 31-35[\mathrm{G}]$

313. Factor S, Podskalny G, Barron K: Persistent neuroleptic-induced rigidity and dystonia in AIDS dementia complex: a clinico-pathological case report. J Neurol Sci 1994; 127:114$120[\mathrm{G}]$

314. Jones GH, Kelly CL, Davies JA: HIV and onset of schizophrenia (letter). Lancet 1987; 1: $982[\mathrm{G}]$

315. Singh A, Goiledge H, Catalan J: Treatment of HIV-related psychotic disorders with risperidone: a series of 21 cases. J Psychosom Res 1997; 42:489-493 [G]

316. Nath A, Jankovic J, Pettigrew LC: Movement disorders and AIDS. Neurology 1987; 37: $37-41[\mathrm{G}]$ 
317. Kieburtz K, Simpson D, Yiannoutsos C, Max MB, Hall CD, Ellis RJ, Marra CM, McKendall R, Singer E, Dal Pan GJ, Clifford DB, Tucker T, Cohen B (AIDS Clinical Trial Group 242 Protocol Team): A randomized trial of amitriptyline and mexiletine for painful neuropathy in HIV infection. Neurology 1998; 51:1682-1688 [A]

318. Janssen RS (American Academy of Neurology Workgroup on the Nomenclature for HIVAssociated Cognitive Disorders): Nomenclature and research case definitions for neurologic manifestations of human immunodeficiency virus-type 1 (HIV-1) infection. Neurology 1991; 41:778-785 [A]

319. Wilson JA, Smith RG: Relation between elderly and AIDS patients with drug-induced Parkinson's disease (letter). Lancet 1987; 2:686 [G]

320. Lera G, Zirulnik J: Pilot study with clozapine in patients with HIV-associated psychosis and drug-induced parkinsonism. Mov Disord 1999; 14:128-131 [B]

321. Fernandez F, Levy JK, Galizzi H: Response of HIV-related depression to psychostimulants: case reports. Hosp Community Psychiatry 1988; 39:628-631 [G]

322. Wagner GJ, Rabkin JG, Rabkin R: Dextroamphetamine as a treatment for depression and low energy in AIDS patients: a pilot study. J Psychosom Res 1997; 42:407-411 [B]

323. Hardy MA, Nardacci D: Does valproate pose a threat to human immunodeficiency virusinfected patients? (letter). J Clin Psychopharmacol 1999; 19:189-190 [G]

324. Maggi JD, Halman MH: The effect of divalproex sodium on viral load: a retrospective review of HIV-positive patients with manic syndromes. Can J Psychiatry 2001; 46:359$362[\mathrm{~F}]$

325. Cozza KL, Swanton EJ, Humphreys CW: Hepatotoxicity with combination of valproic acid, ritonavir, and nevirapine: a case report. Psychosomatics 2000; 41:452-453 [G]

326. Jefferson JW: Possible risks associated with valproate treatment of AIDS-related mania. J Clin Psychiatry 1998; 59:317 [G]

327. Parenti DM, Simon GL, Scheib RG, Meyer WA III, Sztein MB, Paxton H, DiGioia RA, Schulof RS: Effect of lithium carbonate in HIV-infected patients with immune dysfunction. J Acquir Immune Defic Syndr 1988; 1:119-124 [C]

328. Batki SL: Buspirone in drug users with AIDS or AIDS-related complex. J Clin Psychopharmacol 1990; 10(June suppl):111S-115S [G]

329. Trachman SB: Buspirone-induced psychosis in a human immunodeficiency virus-infected man. Psychosomatics 1992; 33:332-335 [G]

330. Frye R: Effect of ritonavir on the pharmacokinetics and pharmacodynamics of alprazolam (abstract). Interscience Conference on Antimicrobial Agents and Chemotherapy 1997 [G]

331. Greenblatt DJ, von Moltke LL, Harmatz JS, Durol AL, Daily JP, Graf JA, Mertzanis P, Hoffman JL, Shader RI: Alprazolam-ritonavir interaction: implications for product labeling. Clin Pharmacol Ther 2000; 67:335-341[A]

332. Chuck SK, Rodvold KA, von Moltke LL, Greenblatt DJ, Shader RI: Pharmacokinetics of protease inhibitors and drug interactions with psychoactive drugs, in Psychological and Public Health Implications of New HIV Therapies. Edited by Ostrow D, Kalichman SC. New York, Plenum, 1998, pp 33-60 [G]

333. Fisher A, Abbatiola M: The effects of oxandrolone on body weight and composition in patients with HIV-associated weight loss (abstract 42351). International Conference on AIDS 1998; $12: 844$ [G]

334. Fisher AE, Abbatiola MM: Effects of oxandrolone on body weight and composition in patients with HIV-associated weight loss (abstract 477). Conference on Retroviruses and Opportunistic Infections 1998; 5:169 [G]

335. Poles MA, Meller JA, Lin A, Weiss WR, Gocke M, Dietrict DT: Oxandrolone as a treatment for AIDS-related weight loss and wasting (abstract 695). Conference on Retroviruses and Opportunistic Infections 1997; 4:193 [G] 
336. Tai VW, Mulligan K, Culp J, Schambelan M: The effects of chronic growth hormone therapy on dietary intake in patients with HIV-associated weight loss (abstract MoB 1388). International Conference on AIDS 1996; 11(1):122 [A]

337. Luna-Castanos G, Osornio L, Gomez DM, Neigo L: Growth hormone in the treatment of weight loss AIDS-related (abstract MoB 1387). International Conference on AIDS 1996; 11(1):122 [G]

338. Gomez CWH, Feregrino-Goyos M, Alvarado-Diez R, Eid-Lidt G, Conde-Mercado JM, Fuentes-Del-Toro S, Mireles MP, Mora-Rodriquez G: Short and long time treatment with growth hormone in AIDS wasting syndrome increase in quality of life and nutritional status (abstract MoB 1386). International Conference on AIDS 1996; 11(1):122 [G]

339. Berger DS, LaMarca A, Landy H, Kaufman RS, Breitmeyer J: A phase III study of recombinant human growth hormone (mammalian cell-derived) in patients with AIDS wasting. International Conference on AIDS 1996; 11:B422 [G]

340. Koster F, Nightingale S, Gesundheit N, Waters D, Bukar J, Qualls C, Danska J, Watson D, Jackson L, Hardy K: A randomized, double-blind, placebo-controlled phase II trial of growth hormone and insulin-like growth factor I for AIDS wasting. Program and Abstracts of the Interscience Conference on Antimicrobial Agents and Chemotherapy 1994; 56 [A]

341. Sharp M, Getty J, Chambers S, Sekeres G: Thalidomide associated weight gain in HIV-1 positive clients (abstract WeB 180). International Conference on AIDS 1996; $20: 112$ [G]

342. Reyes-Teran G, Sierra-Madero JG, Martinez del Cerro V, Arroyo-Figueroa H, Pasquetti A, Calva JJ, Ruiz-Palacios GM: Effects of thalidomide on HIV-associated wasting syndrome: a randomized, double-blind, placebo controlled clinical trial. AIDS 1996; 10:1501-1507 [A]

343. Rabkin JG, Rabkin R, Wagner G: Testosterone replacement therapy in HIV illness. Gen Hosp Psychiatry 1995; 17:37-42 [B]

344. Confrancesco J, Whalen JJ, Dobs AS: Testosterone replacement options for HIV-infected men. J Acquir Immune Defic Syndr Hum Retrovirol 1997; 16:254-265 [F]

345. Chang GWM, Kam PCA: The physiologic and pharmacological roles of cytochrome P450 enzymes. Anaesthesia 1999; 54:42-50 [G]

346. Schwartz EL, Brechbuhl AB, Kahl P, Miller MA, Selwyn P, Friedland GH: Pharmacokinetic interactions of zidovudine and methadone in intravenous drug-using patients with HIV infection. J Acquir Immune Defic Syndr 1992; 5:619-626 [B]

347. Borg L, Kreek M: Clinical problems associated with interactions between methadone pharmacotherapy and medications used in the treatment of HIV-1 positive and AIDS patients. Current Opinion in Psychiatry 1995; 8:199-202 [F]

348. McCance-Katz EF, Rainey PM, Jatlow P, Friedland G (AIDS Clinical Trials Group 262): Methadone effects on zidovudine disposition. J Acquir Immune Defic Syndr Hum Retrovirol 1998; 18:435-443 [A]

349. Rainey P, McCance-Katz EF, Jatlow P, Kosten TR, Friedland G: Opioid effects on zidovudine disposition. NIDA Res Monogr (in press) [G]

350. Sellers E: The pharmacokinetics (PK) of abacavir $(\mathrm{ABC})$ and methadone $(\mathrm{M})$ following coadministration: CNAAL 012 (abstract 663). Program and Abstracts From the 39th Interscience Conference on Antimicrobial Agents and Chemotherapy, 1999 [G]

351. Guibert A, Furlan V, Martino J, Taburet AM: In vitro effect of HIV protease inhibitors on methadone metabolism (abstract A-58). Program and Abstracts From the 37th Interscience Conference of Antimicrobial Agents and Chemotherapy, 1997 [G]

352. McCance-Katz EF, Farber S, Selwyn PA, O’Connor A: Decrease in methadone levels with nelfinavir mesylate (letter). Am J Psychiatry 2000; 157:481 [G]

353. Altice FL, Friedland GH, Cooney EL: Nevirapine induced opiate withdrawal among injection drug users with HIV infection receiving methadone. AIDS 1999; 13:957-962 [D]

354. Piscitelli SC, Burstein AH, Chaitt D, Alfaro RM, Falloon J: Indinavir concentrations and St John's wort. Lancet 2000; 355:547-548 [G] 
355. Winiarski MG: AIDS-Related Psychotherapy. New York, Pergamon Press, 1991 [G]

356. Farber EW, Schwartz JAJ, Shaper PE, Moonen DJ, McDaniel JS: Resilience factors associated with adaptation to HIV disease. Psychosomatics 2000; 41:140-146 [G]

357. Farber EW, McDaniel JS: Assessment and psychotherapy practice implications of new combination antiviral therapies in HIV disease. Professional Psychology: Research and Practice 1999; 30:173-179 [G]

358. Selwyn PA, Arnold R: From fate to tragedy: the changing meanings of life, death, and AIDS. Ann Intern Med 1998; 129:899-902 [G]

359. Robins AG, Dew MA, Davidson S, Penkower L, Becker JT, Kingsley L: Psychosocial factors associated with risky sexual behavior among HIV-seropositive gay men. AIDS Educ Prev 1994; 6:483-492 [G]

360. Aversa SL, Kimberlin C: Psychosocial aspects of antiretroviral medication use among HIV patients. Patient Educ Couns 1996; 29:207-219 [G]

361. Mason HR, Simoni JM, Marks G, Johnson CJ, Richardson JL: Missed opportunities? disclosure of HIV and support seeking among HIV positive African-American and European-American men. AIDS and Behavior 1996; 1:155 [C]

362. Kalichman S, Sikkema K: Psychological sequelae of HIV infection and AIDS: review of empirical findings. Clin Psychol Rev 1994; 14:611-632 [F]

363. Linn JG, Lewis FM, Cain VA, Kimbrough GA: HIV-illness, social support, sense of coherence, and psychosocial well-being in a sample of help-seeking adults. AIDS Educ Prev 1993; 5:254-262 [C]

364. Hall VP: The relationship between social support and health in gay men with HIV/AIDS: an integrative review. J Assoc Nurses AIDS Care 1999; 10:74-86 [E]

365. Leserman J, Perkins DO, Evans DL: Coping with the threat of AIDS: the role of social support. Am J Psychiatry 1992; 149:1514-1520 [C]

366. Gostin LO, Webber DW: HIV infection and AIDS in the public health and health care systems: the role of law and litigation. JAMA 1998; 279:1108-1113 [G]

367. Levy JA, Fox SE: The outreach-assisted model of partner notification with IDUS. Public Health Rep 1998; 113(suppl 1):160-169 [A]

368. West GR, Stark KA: Partner notification for HIV prevention: a critical reexamination. AIDS Educ Prev 1997; 9:68-78 [F]

369. Fenton KA, Peterman TA: HIV partner notification-taking a new look (editorial). AIDS 1997; 11:1535-1546 [G]

370. APA Commission on AIDS: AIDS policy: position statement on confidentiality, disclosure, and protection of others. Am J Psychiatry 1993; 150:852 [G]

371. Bronheim HE, Fulop G, Kunkel EJ, Muskin PR, Schindler BA, Yates WR, Shaw R, Steiner H, Stern TA, Stoudemire A: The Academy of Psychosomatic Medicine practice guidelines for psychiatric consultation in the general medical setting. Psychosomatics 1998; 39:S8-S30 [G]

372. Kalichman SC, Ramachandran B, Ostrow D: Protease inhibitors and the new AIDS combination therapies: implication for psychological services. Professional Psychol Res Practice 1998; 29:349-356 [G]

373. Lucas GM, Chaisson RE, Moore RD: Highly active antiretroviral therapy in a large urban clinic: risk factors for virologic failure and adverse drug reactions. Ann Intern Med 1999; 131:81-87 [D]

374. Wainberg ML, Cournos F: Adherence to treatment. New Dir Ment Health Serv Fall 2000; (87):85-93 [G]

375. McArthur JC GI: HIV Neurocognitive disorders, in The Neurology of AIDS. Edited by Gendelman HE, Lipton SA, Epstein L, Swindells S. New York, Chapman \& Hall, 1996, pp 499-523 [F]

376. Ickovics JR, Meisler AW: Adherence in AIDS clinical trials: a framework for clinical research and clinical care. J Clin Epidemiol 1997; 50:385-391 [G] 
377. Wall TL, Sorensen JL, Batki SL, Delucchi KL, London JA, Chesney MA: Adherence to zidovudine (AZT) among HIV-infected methadone patients: a pilot study of supervised therapy and dispensing compared to usual care. Drug Alcohol Depend 1995; 37:261-269 [B]

378. Sorensen JL, Mascovich A, Wall TL, DePhilippis D, Batki SL, Chesney M: Medication adherence strategies for drug abusers with HIV/AIDS. AIDS Care 1998; 10:297-312 [G]

379. Foudraine NA, Hoetelmans RM, Lange JM, de Wolf F, van Benthem BH, Maas JJ, Keet IP, Portegies P: Cerebrospinal-fluid HIV-1 RNA and drug concentrations after treatment with lamivudine plus zidovudine or stavudine. Lancet 1998; 351:1547-1551 [A]

380. Gulevich SJ, McCutchan JA, Thal LJ, Kirson D, Durand D, Wallace M, Mehta P, Heyes MP, Grant I: Effect of antiretroviral therapy on the cerebrospinal fluid of patients seropositive for the human immunodeficiency virus. J Acquir Immune Defic Syndr 1993; 6:10021007; correction, 1994; 7:994 [B]

381. Goodkin K, Wilkie FL, Concha M, Asthana D, Shapshak P, Douyon R, Fujimura RK, LoPiccolo C: Subtle neuropsychological impairment and minor cognitive-motor disorder in HIV-1 infection: neuroradiological, neurophysiological, neuroimmunological, and virological correlates. Neuroimaging Clin N Am 1997; 7:561-579 [F]

382. Sidtis JJ, Gatsonis C, Price RW, Singer EJ, Collier AC, Richman DD, Hirsch MS, Schaerf FW, Fischl MA, Kieburtz K: Zidovudine treatment of the AIDS dementia complex: results of a placebo-controlled trial. Ann Neurol 1993; 33:343-349 [A]

383. Schmitt FA, Bigley JW, McKinnis R, Logue PE, Evans RW, Drucker JL: Neuropsychological outcome of zidovudine (AZT) treatment of patients with AIDS and AIDS-related complex. N Engl J Med 1988; 319:1573-1578 [A]

384. Azzini M, Nanni S, Astori MR, Brunetto A, Massobrio L: Evaluation of neuropsychiatric parameters in HIV positive subjects treated with zidovudine. Acta Neurol (Napoli) 1990; $12: 36-39[\mathrm{G}]$

385. Vago L, Castagna A, Lazzarin A, Trabattoni G, Cinque P, Costanzi G: Reduced frequency of HIV-induced brain lesions in AIDS patients treated with zidovudine. J Acquir Immune Defic Syndr 1993; 6:42-45 [D]

386. Bell JE, Donaldson YK, Lowrie S, McKenzie CA, Elton RA, Chiswick A, Brettle RP, Ironside JW, Simmonds P: Influence of risk group and zidovudine therapy on the development of HIV encephalitis and cognitive impairment in AIDS patients. AIDS 1996; 10: 493-499 [B]

387. Brew BJ, Bhalla RB, Paul M, Sidtis JJ, Keilp JJ, Sadler AE, Gallardo H, McArthur JC, Schwartz MK, Price RW: Cerebrospinal fluid beta 2-microglobulin in patients with AIDS dementia complex: an expanded series including response to zidovudine treatment. AIDS 1992; 6:461-465 [C]

388. Gorman JM, Mayeux R, Stern Y, Williams JB, Rabkin J, Goetz RR, Ehrhardt AA: The effect of zidovudine on neuropsychiatric measures in HIV-infected men. Am J Psychiatry 1993; 150:505-507 [B]

389. Yerly S, Kaiser L, Race E, Bru JP, Clavel F, Perrin L: Transmission of antiretroviral-drugresistant HIV-1 variants. Lancet 1999; 354:729-733 [G]

390. De Ronchi D, Lazzari C, Rucci P, Cangialosi A, Volterra V: Neurocognitive effects of zidovudine and 2',3'-dideoxyinosine during the treatment of asymptomatic and symptomatic HIV-1 seropositive patients: comparison with non-treated patients. Human Psychopharmacology 1996; 11:415-420 [G]

391. Brew BJ, Brown SJ, Catalan J: Safety and efficacy of abacavir (ABC, 1592) in AIDS dementia complex (study CNAB 3001). Abstracts of the 12th International Conference on AIDS. Geneva, International AIDS Society, 1998, p 559 [B]

392. Thomas SA, Segal MB: The transport of the anti-HIV drug, 2',3'-didehydro-3'-deoxythymidine (D4T), across the blood-brain and blood-cerebrospinal fluid barriers. Br J Pharmacol 1998; 125:49-54 [G] 
393. Stahle L, Martin C, Svensson JO, Sonnerborg A: Indinavir in cerebrospinal fluid of HIV1-infected patients (letter). Lancet 1997; 350:1823 [G]

394. Ferrando S, van Gorp W, McElhiney M, Gogin K, Sewell M, Rabkin J: Highly active antiretroviral treatment in HIV infection: benefits for neuropsychological function. AIDS 1998; 12:F65-F70 [B]

395. Moore RD, Keruly J, Richman DD, Creagh-Kirk T, Chaisson RE: Natural history of advanced HIV disease in patients treated with zidovudine. AIDS 1992; 6:671-677 [B]

396. Montforte A, Musicco M, Galli M: Italian multicenter study of didanosine: compassionate use in advanced HIV infection. Eur J Microb Infect Dis 1997; 16:135-142 [G]

397. Day JJ, Grant I, Atkinson JH, Brysk LT, McCutchan JA, Hesselink JR, Heaton RK, Weinrich JD, Spector SA, Richman DD: Incidence of AIDS dementia in a two-year followup of AIDS and ARC patients on an initial phase II AZT placebo-controlled study: San Diego cohort. J Neuropsychiatry Clin Neurosci 1992; 4:15-20 [A]

398. Heseltine PN, Goodkin K, Atkinson JH, Vitiello B, Rochon J, Heaton RK, Eaton EM, Wilkie FL, Sobel E, Brown SJ, Feaster D, Schneider L, Goldschmidts WL, Stover ES: Randomized double-blind placebo-controlled trial of peptide T for HIV-associated cognitive impairment. Arch Neurol 1998; 55:41-51 [A]

399. Lipton SA: HIV-related neuronal injury: potential therapeutic intervention with calcium channel antagonists and NMDA antagonists. Mol Neurobiol 1994; 8:181-196 [F]

400. Mapou RL, Law WA, Wagner K, Malone JL, Skillman DR: Neuropsychological effects of interferon alfa-n3 treatment in asymptomatic human immunodeficiency virus-1-infected individuals. J Neuropsychiatry Clin Neurosci 1996; 8:74-81 [B]

401. Skillman DR, Malone JL, Decker CF, Wagner KF, Mapou RL, Liao MJ, Testa D, Meltzer MS: Phase I trial of interferon alfa-n 3 in early-stage human immunodeficiency virus type 1 disease: evidence for drug safety, tolerance, and antiviral activity. J Infect Dis 1996; 173: $1107-1114$ [B]

402. Galgani S, Balestra P, Narciso P, Tozzi V, Sette P, Pau F, Visco G: Nimodipine plus zidovudine versus zidovudine alone in the treatment of HIV-1-associated cognitive deficits (letter). AIDS 1997; 11:1520-1521 [G]

403. Navia BA, Dafni U, Simpson D, Tucker T, Singer E, McArthur JC, Yiannoutsos C, Zaborski L, Lipton SA: A phase I/II trial of nimodipine for HIV-related neurologic complications. Neurology 1998; 51:221-228 [A]

404. van Dyck CH, McMahon TJ, Rosen MI, O'Malley SS, O'Connor PG, Lin CH, Pearsall HR, Woods SW, Kosten TR: Sustained-release methylphenidate for cognitive impairment in HIV1-infected drug abusers: a pilot study. J Neuropsychiatry Clin Neurosci 1997; 9:29-36 [A]

405. Holmes VF, Fernandez F, Levy JK: Psychostimulant response in AIDS-related complex patients. J Clin Psychiatry 1989; 50:5-8 [B]

406. Dana Consortium on the Therapy of HIV Dementia and Related Cognitive Disorders: A randomized, double-blind, placebo-controlled trial of deprenyl and thioctic acid in human immunodeficiency virus-associated cognitive impairment. Neurology 1998; 50:645-651 [A]

407. Gisslen M, Larsson M, Norkrans G, Fuchs D, Wachter H, Hagberg L: Tryptophan concentrations increase in cerebrospinal fluid and blood after zidovudine treatment in patients with HIV type 1 infection. AIDS Res Hum Retroviruses 1994; 10:947-951 [B]

408. Herzlich BC, Schiano TD: Reversal of apparent AIDS dementia complex following treatment with vitamin B12. J Intern Med 1993; 233:495-497 [G]

409. Adams F, Fernandez F, Andersson BS: Emergency pharmacotherapy in the critically ill cancer patient. Psychosomatics 1986; 27(Jan suppl):33-38 [G]

410. Muskin PR, Mellman LA, Kornfeld DS: A "new" drug for treating agitation and psychosis in the general hospital: chlorpromazine. Gen Hosp Psychiatry 1986; 8:404-410 [G]

411. Trzepacz PT, Baker RW, Greenhouse J: A symptom rating scale for delirium. Psychiatry Res $1988 ; 23: 89-97[G]$ 
412. Resnick M, Burton BT: Droperidol vs haloperidol in the initial management of acutely agitated patients. J Clin Psychiatry 1984; 45:298-299 [A]

413. Thomas H Jr, Schwartz E, Petrilli R: Droperidol versus haloperidol for chemical restraint of agitated and combative patients. Ann Emerg Med 1992; 21:407-413 [A]

414. Wagner G, Maguen S, Rabkin J: Ethnic differences in response to fluoxetine in a controlled trial with depressed HIV-positive patients. Psychiatr Serv 1998; 49:239-240 [A]

415. Cazzulio CL, Bessone E, Bertrando P, Pedrazzoli L, Cusini M: Treatment of depression in HIV-infected patients. J Psychiatry Neurosci 1998; 23:293-297 [B]

416. Levine S, Anderson D, Bystritsky A, Baron D: A report of eight HIV-seropositive patients with major depression responding to fluoxetine. J Acquir Immune Defic Syndr 1990; 3: 1074-1077 [B]

417. Gottlieb JF: book review, A Lazare (ed): Outpatient Psychiatry: Diagnosis and Treatment, 2nd ed. Am J Psychiatry 1991; 148:805-806 [G]

418. Grassi B, Gambini O, Garghentini G, Lazzarin A, Scarone S: Efficacy of paroxetine for the treatment of depression in the context of HIV infection. Pharmacopsychiatry 1997; 30:70-71 [B]

419. Ferrando SJ, Rabkin JG, de Moore GM, Rabkin R: Antidepressant treatment of depression in HIV-seropositive women. J Clin Psychiatry 1999; 60:741-756 [B]

420. Fernandez F, Levy J: Efficacy of venlafaxine in HIV-depressive disorders. Psychosomatics 1997; 38:173-174 [B]

421. White J, Christensen J, Clifford M: Methylphenidate as a treatment for depression in acquired immunodeficiency syndrome: an n-of-1 trial. J Clin Psychiatry 1992; 53:153-156 [A]

422. Walling V, Pfefferbaum B: The use of methylphenidate in a depressed adolescent with AIDS. J Dev Behav Pediatr 1990; 11:195-197 [G]

423. Perkins D, Evans DL: HIV-related major depression: response to zidovudine treatment. Psychosomatics 1991; 32:451-454 [G]

424. Schaerf F, Miller RR, Lipsey JR, McPherson RW: ECT for major depression in four patients infected with human immunodeficiency. Am J Psychiatry 1989; 146:782-784 [G]

425. Targ E, Karasic D, Diefenbach P, Anderson D, Bystritsky A, Fawzy F: Structured group therapy and fluoxetine to treat depression in HIV-positive persons. Psychosomatics 1994; 35:132-137 [A]

426. Markowitz JC, Kocsis JH, Fishman B, Spielman LA, Jacobsberg LB, Frances AJ, Klerman GL, Perry SW: Treatment of depressive symptoms in human immunodeficiency viruspositive patients. Arch Gen Psychiatry 1998; 55:452-457 [A]

427. Markowitz JC, Klerman GL, Perry SW: Interpersonal psychotherapy of depressed HIVpositive outpatients. Hosp Community Psychiatry 1992; 43:885-890 [B]

428. Levine S, Bystritsky A, Baron D, Jones L: Group psychotherapy for HIV-seropositive patients with major depression. Am J Psychother 1991; 45:413-424 [B]

429. Treisman G: HIV, AIDS, and the Brain, vol 72. New York, Raven Press, 1994 [G]

430. Lyketsos C, Fishman M, Hutton H, Cox T, Hobbs S, Spoler C, Hunt W, Driscoll J, Treisman G: The effectiveness of psychiatric treatment for HIV-infected patients. Psychosomatics 1997: 38:423-432 [B]

431. Kelly JA, Murphy DA, Bahr GR, Kalichman SC, Morgan MG, Stevenson LY, Koob JJ, Brasfield TL, Bernstein BM: Outcome of cognitive-behavioral and support group brief therapies for depressed, HIV-infected persons. Am J Psychiatry 1993; 150:1679-1686 [A]

432. Eller L: Effects of two cognitive-behavioral interventions on immunity and symptoms in persons with HIV. Ann Behav Med 1995; 17:339-348 [B]

433. Halman MH, Worth JL, Sanders KM, Renshaw PF, Murray GB: Anticonvulsant use in the treatment of manic syndromes in patients with HIV-1 infection. J Neuropsychiatry Clin Neurosci 1993; 5:430-434 [D]

434. RachBeisel JA, Weintraub E: Valproic acid treatment of AIDS-related mania (letter). J Clin Psychiatry 1997; 58:406-407 [G] 
435. Guydish J, Temoshok L, Dilley J, Rinaldi J: Evaluation of a hospital based substance abuse intervention and referral service for HIV affected patients. Gen Hosp Psychiatry 1990; 12:1-7 [B]

436. Sorensen J, Batki S, Good P, Wilkinson K: Methadone maintenance program for AIDSaffected opiate addicts. J Subst Abuse Treat 1989; 6:87-94 [G]

437. Ferrando SJ, Batki SL: HIV-infected intravenous drug users in methadone maintenance treatment: clinical problems and their management. J Psychoactive Drugs 1991; 23:217$224[\mathrm{G}]$

438. Batki SL: Abstract. International Conference on AIDS 1995 [B]

439. Antela A, Casado JL, Gonzalez MJ, Perez P, Perez-Elias MJ, Montilla P, Buzon L: Influence of a methadone maintenance programme on the improved outcome of a cohort of injecting drug users with advanced HIV disease (letter). AIDS 1997; 11:1405-1406 [G]

440. Batki SL: Abstract. International Conference on AIDS 1993 [A]

441. McDaniel JS, Blalock A: Diagnosis and management of HIV-related mood and anxiety disorders. New Directions in Psychiatric Services (in press) [F]

442. McDaniel J, Johnson K: Obsessive-compulsive disorder in HIV disease: response to fluoxetine. Psychosomatics 1995; 36:147-150 [G]

443. Rahman M, Gregory R: Trichotillomania associated with HIV infection in response to sertraline. Psychosomatics 1995; 36:417-418 [G]

444. Perretta P, Nisita C, Zaccagnini E, Scasso A, Nuccorini A, Santa M, Cassano G: Diagnosis and clinical use of bromperidol in HIV-related psychoses in a sample of seropositive patients with brain damage. Int Clin Psychopharmacol 1992; 7:95-99 [B]

445. Scamvougeras A, Rosebush PI: AIDS-related psychosis with catatonia responding to lowdose lorazepam. J Clin Psychiatry 1992; 53:414-415 [G]

446. Snyder S, Prenzlauer S, Maruyama N, Rose DN: Catatonia in a patient with AIDS-related dementia (letter). J Clin Psychiatry 1992; 53:414 [G]

447. El-Mallakh R: AIDS dementia-related psychosis: is there a window of vulnerability? AIDS Care 1992; 4:381-387 [F]

448. Norman SE, Chediak AD, Kiel M, Cohn MA: Sleep disturbances in HIV-infected homosexual men. AIDS 1990; 4:775-781 [C]

449. Norman SE, Chediak AD, Freeman C, Kiel M, Mendez A, Duncan R, Simoneau J, Nolan B: Sleep disturbances in men with asymptomatic human immunodeficiency (HIV) infection. Sleep 1992; 15:150-155 [C]

450. Darko DF, McCutchan JA, Kripke DF, Gillin JC, Golshan S: Fatigue, sleep disturbance, disability, and indices of progression of HIV infection. Am J Psychiatry 1992; 149:514-520 [C]

451. Darko DF, Mitler MM, Miller JC: Growth hormone, fatigue, poor sleep, and disability in HIV infection. Neuroendocrinology 1998; 67:317-324 [C]

452. Epstein LJ, Strollo PJ Jr, Donegan RB, Delmar J, Hendrix C, Westbrook PR: Obstructive sleep apnea in patients with human immunodeficiency virus (HIV) disease. Sleep 1995; $18: 368-376[\mathrm{C}]$

453. Hansen M, Kubicki S, Henkes H, Terstegge G, Scholz, Weiss R: The effects of flurazepam on sleep in AIDS patients. New Trends in Clinical Neuropharmacology 1989; 3:134-135 [B]

454. Pizzo PA, Eddy J, Falloon J, Balis FM, Murphy RF, Moss H, Wolters P, Brouwers P, Jarosinski P, Rubin M: Effect of continuous intravenous infusion of zidovudine (AZT) in children with symptomatic HIV infection. N Engl J Med 1988; 319:889-896 [B]

455. Brouwers P, Moss H, Wolters P, Eddy J, Balis F, Poplack D, Pizzo P: Effect of continuousinfusion zidovudine therapy on neuropsychologic functioning in children with symptomatic human immunodeficiency virus infection. J Pediatr 117:980-985 [B]

456. Nozyce M, Hoberman M, Arpadi S, Wiznia A, Lambert G, Dobroszycki J, Chang CJ, St Louis Y: A 12-month study of the effects of oral zidovudine on neurodevelopmental functioning in a cohort of vertically HIV-infected inner-city children. AIDS 1994; 8:635$639[\mathrm{~B}]$ 
457. Raskino C, Pearson DA, Baker CJ, Lifschitz MH, O'Donnell K, Mintz M, Nozyce M, Bruwors P, McKinney RE, Jimenez E, England JA (Pediatric AIDS Clinical Trials Group 152 Study Team): Neurologic, neurocognitive, and brain growth outcomes in human immunodeficiency virus-infected children receiving different nucleoside antiretroviral regimens. Pediatrics 1999; 104(3):e32 [A]

458. Tepper VJ, Farley JJ, Rothman MI, Houck DL, Davis KF, Collins-Jones TL, Wachtel RC: Neurodevelopmental/neuroradiologic recovery of a child infected with HIV after treatment with combination antiretroviral therapy using the HIV-specific protease inhibitor ritonavir. Pediatrics 1998; 101:e7 [G]

459. Cesena M, Lee D, Cebollero A, Steingard R: Case study: behavioral symptoms of pediatric HIV-1 encephalopathy successfully treated with clonidine. J Am Acad Child Adolesc Psychiatry 1995; 34:302-306 [G]

460. Anand A, Carmosino L, Glatt A: Management of recalcitrant pain in a pediatric acquired immunodeficiency syndrome patient. Pediatr Infect Dis J 1993; 12:159-160 [G]

461. Hirschfeld S, Moss H, Dragisic K, Smith W, Pizzo PA: Pain in pediatric human immunodeficiency virus infection: incidence and characteristics in a single-institution pilot study. Pediatrics 1996; 98:449-452 [G]

462. Grinspoon S, Corcoran C, Askari H, Schoenfeld D, Wolf L, Burrows B, Walsh M, Hayden D, Parlman K, Anderson E, Basgoz N, Klibanski A: Effects of androgen administration in men with the AIDS wasting syndrome: a randomized, double-blind, placebo-controlled trial. Ann Intern Med 1998; 129:18-26 [A]

463. Rabkin JG, Rabkin R, Wagner GJ: Testosterone treatment of clinical hypogonadism in patients with HIV/AIDS. Int J STD AIDS 1997; 8:537-545 [F]

464. Hengge UR, Baumann M, Maleba R, Brockmeyer NH, Goos M: Oxymetholone promotes weight gain in patients with advanced human immunodeficiency virus (HIV-1) infection. Br J Nutr 1996; 75:129-138 [A]

465. Miller K, Corcoran C, Armstrong C, Caramelli K, Anderson E, Cotton D, Basgoz N, Hirschhorn L, Tuomala R, Schoenfeld D, Daugherty C, Mazer N, Grinspoon S: Transdermal testosterone administration in women with acquired immunodeficiency syndrome wasting: a pilot study. J Clin Endocrinol Metab 1998; 83:2717-2725 [B]

466. Wagner G, Rabkin J, Rabkin R: Exercise as a mediator of psychological and nutritional effects of testosterone therapy in HIV+ men. Med Sci Sports Exerc 1998; 30:811-817 [B]

467. Strawford A, Barbieri T, Van Loan M, Parks E, Catlin D, Barton N, Neese R, Christiansen M, King J, Hellerstein MK: Resistance exercise and supraphysiologic androgen therapy in eugonadal men with HIV-related weight loss: a randomized controlled trial. JAMA 1999; 281:1282-1290 [A]

468. Breitbart WS, Rosenfeld B, Kaim M, Funesti-Esch J: Psychostimulants for fatigue in the HIV+ patient: preliminary findings of a placebo-controlled trial of methylphenidate vs pemoline. Psychosomatics 1999; 40:160-161 [A]

469. Rabkin JG, Ferrando SJ, Wagner GJ, Rabkin R: DHEA treatment for HIV+ patients: effects on mood, androgenic and anabolic parameters. Psychoneuroendocrinology 2000; 25:53-68 [B]

470. Holzemer WL, Henry SB, Reilly CA: Assessing and managing pain in AIDS care: the patient perspective. J Assoc Nurses AIDS Care 1998; 9:22-30 [G]

471. Kemper CA, Kent G, Burton S, Deresinski SC: Mexiletine for HIV-infected patients with painful peripheral neuropathy: a double-blind, placebo-controlled, crossover treatment trial. J Acquir Immune Defic Syndr Hum Retrovirol 1998; 19:367-372 [A]

472. Shlay JC, Chaloner K, Max MB, Flaws B, Reichelderfer P, Wentworth D, Hillman S, Brizz B, Cohn DL: Acupuncture and amitriptyline for pain due to HIV-related peripheral neuropathy: a randomized controlled trial: Terry Beirn Community Programs for Clinical Research on AIDS. JAMA 1998; 280:1590-1595 [A]

473. Hall MC, Ahmad S: Interaction between sildenafil and HIV-1 combination therapy (letter). Lancet 1999; 353:2071-2072 [G]

\section{6}

APA Practice Guidelines

Copyright 2010, American Psychiatric Association. APA makes this practice guideline freely available to promote its dissemination and use; however, copyright protections are enforced in full. No part of this guideline may be reproduced except as permitted under Sections 107 and 108 of U.S. Copyright Act. For permission for reuse, visit APPI Permissions \& Licensing Center at http://www.appi.org/CustomerService/Pages/Permissions.aspx. 